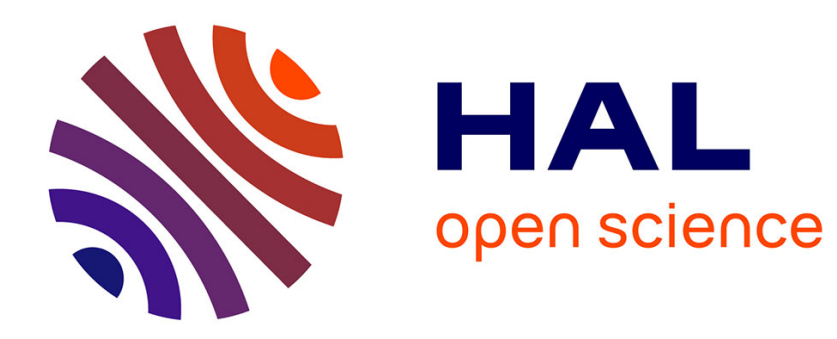

\title{
Estimates of the Linearization of Circle Diffeomorphisms
}

Mostapha Benhenda

\section{To cite this version:}

Mostapha Benhenda. Estimates of the Linearization of Circle Diffeomorphisms. 2011. hal-00628297

\section{HAL Id: hal-00628297 \\ https://hal.science/hal-00628297}

Preprint submitted on 2 Oct 2011

HAL is a multi-disciplinary open access archive for the deposit and dissemination of scientific research documents, whether they are published or not. The documents may come from teaching and research institutions in France or abroad, or from public or private research centers.
L'archive ouverte pluridisciplinaire HAL, est destinée au dépôt et à la diffusion de documents scientifiques de niveau recherche, publiés ou non, émanant des établissements d'enseignement et de recherche français ou étrangers, des laboratoires publics ou privés. 


\title{
Estimates of the Linearization of Circle Diffeomorphisms
}

\author{
Mostapha Benhenda*
}

September 30, 2011

\begin{abstract}
A celebrated theorem by Herman and Yoccoz asserts that if the rotation number $\alpha$ of a $C^{\infty}$-diffeomorphism of the circle $f$ satisfies a Diophantine condition, then $f$ is $C^{\infty}$-conjugated to a rotation. In this paper, we establish explicit relationships between the $C^{k}$ norms of this conjugacy and the Diophantine condition on $\alpha$. To obtain these estimates, we follow a suitably modified version of Yoccoz's proof.
\end{abstract}

\section{Introduction}

In his seminal work, M. Herman [5] shows the existence of a set $A$ of Diophantine numbers of full Lebesgue measure such that for any rotation number $\alpha \in A$ of a circle diffeomorphism $f$ of class $C^{\omega}$ (resp. $C^{\infty}$ ), there is a $C^{\omega}$-diffeomorphism (resp. $C^{\infty}$-diffeomorphism) $h$ such that $h f h^{-1}=R_{\alpha}$. In the $C^{\infty}$ case, J. C. Yoccoz [14] extended this result to all Diophantine rotation numbers. Results in analytic class and in finite differentiability class subsequently enriched the global theory of circle diffeomorphisms $[11,9,8,13,7,15,4,10]$. In the perturbative theory, KAM theorems usually provide a bound on the norm of the conjugacy that involves the norm of the perturbation and the Diophantine constants of the number $\alpha$ (see [5, 12, 3] for example). We place ourselves in the global setting, we compute a bound on the norms of this conjugacy $h$ in function of $k,|D f|_{0}, W(f),|S f|_{k-3}, \beta$ and $C_{d}$.

To obtain these estimates, we follow a suitably modified version of Yoccoz's proof. Indeed, Yoccoz's proof needs to be modified because a priori, it does not exclude the fact that the set:

$E_{X}=\left\{|D h|_{0} / \exists f \in \operatorname{Diff}_{+}^{k}\left(\mathbb{T}^{1}\right), f=h^{-1} R_{\alpha} h, \alpha \in D C\left(\beta, C_{d}\right), \max \left(k, \beta, C_{d},|D f|_{0}, W(f),|S f|_{k-3}\right) \leq X\right\}$

could be unbounded for any fixed $X>0$.

These estimates have natural applications to the global study of circle diffeomorphisms with Liouville rotation number: in [2], they allow to show the following results: 1) Given a diffeomorphism $f$ of rotation number $\alpha$, for a Baire-dense set of $\alpha$, it is possible to accumulate $R_{\alpha}$ with a sequence $h_{n} f h_{n}^{-1}, h_{n}$ being a diffeomorphism. 2) Given two commuting diffeomorphisms $f$ and $g$, with the rotation number $\alpha$ of $f$ belonging to a specified Baire-dense set, it is possible to approach each of them by commuting diffeomorphisms $f_{n}$ and $g_{n}$ that are differentiably conjugated to rotations.

${ }^{*}$ Laboratoire d'Analyse, Geometrie et Applications, Paris 13 University, 99 Avenue J.B. Clement, 93430 Villetaneuse, France. Contact: mostaphabenhenda@gmail.com 


\subsection{Notations}

We follow the notations of [14].

- The circle is noted $\mathbb{T}^{1}$. The group of orientation-preserving circle diffeomorphisms of class $C^{r}$ is denoted $\operatorname{Diff}_{+}^{r}\left(\mathbb{T}^{1}\right)$. The group of $\mathbb{Z}$-periodic diffeomorphisms of class $C^{r}$ of the real line is noted $C^{r}\left(\mathbb{T}^{1}\right)$. We often work in the universal cover $D^{r}\left(\mathbb{T}^{1}\right)$, which is the group of diffeomorphisms $f$ of class $C^{r}$ of the real line such that $f-I d \in C^{r}\left(\mathbb{T}^{1}\right)$. Note that if $f \in D^{r}\left(\mathbb{T}^{1}\right)$ and $r \geq 1$, then Df $\in C^{r-1}\left(\mathbb{T}^{1}\right)$.

- The derivative of $f \in D^{1}\left(\mathbb{T}^{1}\right)$ is noted $D f$. The Schwartzian derivative $S f$ of $f \in D^{3}\left(\mathbb{T}^{1}\right)$ is defined by:

$$
S f=D^{2} \log D f-\frac{1}{2}(D \log D f)^{2}
$$

- The total variation of the logarithm of the first derivative of $f$ is:

$$
W(f)=\sup _{a_{0} \leq \ldots \leq a_{n}} \sum_{i=0}^{n}\left|\log D f\left(a_{i+1}\right)-\log D f\left(a_{i}\right)\right|
$$

- For any continuous and $\mathbb{Z}$-periodic function $\phi$, let:

$$
|\phi|_{0}=\|\phi\|_{0}=\sup _{x \in \mathbb{R}}|\phi(x)|
$$

- Let $0<\gamma^{\prime}<1 . \phi \in C^{0}\left(\mathbb{T}^{1}\right)$ is Holder of order $\gamma^{\prime}$ if:

$$
|\phi|_{\gamma^{\prime}}=\sup _{x \neq y} \frac{|\phi(x)-\phi(y)|}{|x-y|^{\gamma^{\prime}}}<+\infty
$$

Let $\gamma \geq 1$ be a real number. In all the paper, we write $\gamma=r+\gamma^{\prime}$ with $r \in \mathbb{N}$ and $0 \leq \gamma^{\prime}<1$.

- A function $\phi \in C^{r}\left(\mathbb{T}^{1}\right)$ is said to be of class $C^{\gamma}$ if $D^{r} \phi \in C^{\gamma^{\prime}}\left(\mathbb{T}^{1}\right)$. The space of these functions is noted $C^{\gamma}\left(\mathbb{T}^{1}\right)$ and is given the norm:

$$
\|\phi\|_{\gamma}=\max \left(\max _{0 \leq j \leq r}\left\|D^{j} \phi\right\|_{0},\left|D^{r} \phi\right|_{\gamma^{\prime}}\right)
$$

If $\gamma=0$ or $\gamma \geq 1$, the $C^{\gamma}$-norm of $\phi$ is indifferently denoted $\|\phi\|_{\gamma}$ or $|\phi|_{\gamma}$. Thus, when possible, we favor the simpler notation $|\phi|_{\gamma}$.

- For $\alpha \in \mathbb{R}$ (respectively, $\alpha \in \mathbb{T}^{1}$ ), we denote $R_{\alpha} \in D^{\infty}\left(\mathbb{T}^{1}\right)$ (respectively, $R_{\alpha} \in$ $\left.\operatorname{Diff}_{+}^{\infty}\left(\mathbb{T}^{1}\right)\right)$. the map $x \mapsto x+\alpha$.

- An irrational number $\alpha \in D C\left(C_{d}, \beta\right)$ satisfies a Diophantine condition of order $\beta \geq 0$ and constant $C_{d}>0$ if for any rational number $p / q$, we have:

$$
\left|\alpha-\frac{p}{q}\right| \geq \frac{C_{d}}{q^{2+\beta}}
$$

Moreover, if $\beta=0$, then $\alpha$ is of constant type $C_{d}$. 
- Let $\alpha_{-2}=\alpha, \alpha_{-1}=1$. For $n \geq 0$, we define a real number $\alpha_{n}$ (the Gauss sequence of $\alpha$ ) and an integer $a_{n}$ by the relations $0<\alpha_{n}<\alpha_{n-1}$ and

$$
\alpha_{n-2}=a_{n} \alpha_{n-1}+\alpha_{n}
$$

- In the following statements, $C_{i}[a, b, \ldots]$ denotes a positive numerical function of real variables $a, b, \ldots$, with an explicit formula that we compute.

$C[a, b, \ldots]$ denotes a numerical function of $a, b, \ldots$, with an explicit formula that we do not compute.

- We use the notations $a \wedge b=a^{b}, e^{(n)} \wedge x$ the $n^{\text {th }}$ - iterate of $x \mapsto \exp x,\lfloor x\rfloor$ for the largest integer such that $\lfloor x\rfloor \leq x$, and $\lceil x\rceil$ for the smallest integer such that $\lceil x\rceil \geq x$.

We recall Yoccoz's theorem [14]:

Theorem 1.1. Let $k \geq 3$ an integer and $f \in D^{k}\left(\mathbb{T}^{1}\right)$. We suppose that the rotation number $\alpha$ of $f$ is Diophantine of order $\beta$. If $k>2 \beta+1$, there exists a diffeomorphism $h \in D^{1}\left(\mathbb{T}^{1}\right)$ conjugating $f$ to $R_{\alpha}$. Moreover, for any $\eta>0, h$ is of class $C^{k-1-\beta-\eta}$.

\subsection{Statement of the results}

\subsection{1 $C^{1}$ estimations}

Theorem 1.2. Let $f \in D^{3}\left(\mathbb{T}^{1}\right)$ of rotation number $\alpha$, such that $\alpha$ is of constant type $C_{d}$. There exists a diffeomorphism $h \in D^{1}\left(\mathbb{T}^{1}\right)$ conjugating $f$ to $R_{\alpha}$, which satisfies the estimation:

$$
|D h|_{0} \leq e \wedge\left(\frac{C_{1}\left[W(f),|S f|_{0}\right]}{C_{d}}\right)
$$

The expression of $C_{1}$ is given page 10.

More generally, for a Diophantine rotation number $\alpha \in D C\left(C_{d}, \beta\right)$, we have:

Theorem 1.3. Let $k \geq 3$ be an integer and $f \in D^{k}\left(\mathbb{T}^{1}\right)$. Let $\alpha \in D C\left(C_{d}, \beta\right)$ be the rotation number of $f$. If $k>2 \beta+1$, there exists a diffeomorphism $h \in D^{1}\left(\mathbb{T}^{1}\right)$ conjugating $f$ to $R_{\alpha}$, which satisfies the estimation:

$$
|D h|_{0} \leq C_{2}\left[k, \beta, C_{d},|D f|_{0}, W(f),|S f|_{k-3}\right]
$$

The expression of $C_{2}$ is given page 23 .

Moreover, if $k \geq 3 \beta+9 / 2$, we have:

$$
|D h|_{0} \leq e^{(3)} \wedge\left(C_{3}[\beta] C_{4}\left[C_{d}\right] C_{5}\left[|D f|_{0}, W(f),|S f|_{0}\right] C_{6}\left[|S f|_{[3 \beta+3 / 2]}\right]\right)
$$

The expressions of $C_{3}, C_{4}, C_{5}, C_{6}$ are given page 28 .

Let $\delta=k-2 \beta-1$. When $\delta \rightarrow 0$, we have:

$|D h|_{0} \leq e^{(3)} \wedge\left(\frac{1}{\delta^{2}} C_{7}\left[k, C_{d},|D f|_{0}, W(f),|S f|_{0}\right]+\frac{C[\delta]}{\delta^{2}} C\left[k, C_{d},|D f|_{0}, W(f),|S f|_{0},|S f|_{k-3}\right]\right)$

where $C[\delta] \rightarrow_{\delta \rightarrow 0} 0$. The expression of $C_{7}$ is given page 30 . 
Remark 1.4. Katznelson and Ornstein [9] showed that the assumption $k>2 \beta+1$ in Yoccoz's theorem is not optimal (instead it is $k>\beta+2$ ). Therefore, the divergence of the bound given by estimation (3) is because we compute the bound of the conjugacy by following the Herman-Yoccoz method.

Remark 1.5. Let $\alpha_{n}$ be the Gauss sequence associated with $\alpha$. Yoccoz's proof already gives the following result: if $k \geq 3 \beta+9 / 2$ and if, for any $n \geq 0$,

$$
\frac{\alpha_{n+1}}{\alpha_{n}} \geq C_{8}\left[n, k, W(f),|S f|_{k-3}\right]
$$

then:

$$
|D h|_{0} \leq \exp \left(C_{9}\left[k, W(f),|S f|_{k-3}\right]^{C_{10}(\beta)}\right)|D f|_{0}^{2}
$$

The expressions of $C_{8}, C_{9}, C_{10}$ are given page 30 .

\subsection{2 $C^{u}$ estimations}

Theorem 1.6. Let $k \geq 3$ an integer, $\eta>0$ and $f \in D^{k}\left(\mathbb{T}^{1}\right)$. Let $\alpha \in D C\left(C_{d}, \beta\right)$ be the rotation number of $f$. If $k>2 \beta+1$, there exists a diffeomorphism $h \in D^{k-1-\beta-\eta}\left(\mathbb{T}^{1}\right)$ conjugating $f$ to $R_{\alpha}$, which satisfies the estimation:

$$
\|D h\|_{k-2-\beta-\eta} \leq e^{([\log ((k-2-\beta) / \eta) / \log (1+1 /(2 \beta+3))])} \wedge\left(C_{11}\left[\eta, k, \beta, C_{d},|D f|_{0}, W(f),|S f|_{k-3}\right]\right)
$$

The expression of $C_{11}$ is given page 48.

Moreover, if $k \geq 3 \beta+9 / 2$, we have:

$$
\|D h\|_{\frac{k}{2(\beta+2)}-\frac{1}{2}} \leq e \wedge\left(C_{12}[k] e^{(2)} \wedge\left(2+C_{3}[\beta] C_{4}\left[C_{d}\right] C_{5}\left[|D f|_{0}, W(f),|S f|_{0}\right] C_{6}\left[|S f|_{k-3}\right]\right)\right)
$$

The expression of $C_{12}$ is given page 46.

If $\alpha$ is of constant type, for any $k>3$, we have:

$$
\|D h\|_{\frac{k}{4}-\frac{1}{2}} \leq e \wedge\left(C_{13}[k]\left[C_{14}\left[W(f),|S f|_{k-3}\right]+\frac{C_{1}\left[W(f),|S f|_{0}\right]}{C_{d}}\right]^{4}\right)
$$

The expressions of $C_{13}$ and $C_{14}$ are given page 47.

\section{Preliminaries}

Let $f \in D^{0}\left(\mathbb{T}^{1}\right)$ be a homeomorphism and $x \in \mathbb{R}$. When $n$ tends towards infinity, $\left(f^{n}(x)-x\right) / n$ admits a limit independent of $x$, noted $\rho(f)$. We call it the translation number of $f$. Two lifts of $f \in \operatorname{Diff}_{+}^{0}\left(\mathbb{T}^{1}\right)$ only differ by a constant integer, so this is also the case for their translation numbers. We call the class of $\rho(f) \bmod \mathbb{Z}$ the rotation number of $f$. We still denote it $\rho(f)$. It is invariant by conjugacy. Let $f \in D^{2}\left(\mathbb{T}^{1}\right)$. When $\alpha=\rho(f)$ is irrational, Denjoy showed that $f$ is topologically conjugated to $R_{\alpha}$. However, this conjugacy is not always differentiable (see $[1,5,7,15])$. The regularity 
of this conjugacy depends on the Diophantine properties of the rotation number $\alpha$ (see Yoccoz's theorem 1.1).

Let $\alpha$ be an irrational number. Let the distance of $\alpha$ to the closest integer be:

$$
\|\alpha\|=\inf _{p \in \mathbb{Z}}|\alpha-p|
$$

For $n \geq 1, a_{n} \geq 1$. Let $\alpha=a_{0}+1 /\left(a_{1}+1 /\left(a_{2}+\ldots\right)\right)$ be the development of $\alpha$ in continued fraction. We denote it $\alpha=\left[a_{0}, a_{1}, a_{2}, \ldots\right]$. Let $p_{-2}=q_{-1}=0, p_{-1}=q_{-2}=1$. For $n \geq 0$, let $p_{n}$ and $q_{n}$ be:

$$
\begin{aligned}
& p_{n}=a_{n} p_{n-1}+p_{n-2} \\
& q_{n}=a_{n} q_{n-1}+q_{n-2}
\end{aligned}
$$

We have $q_{0}=1, q_{n} \geq 1$ for $n \geq 1$. The rationals $p_{n} / q_{n}$ are called the convergents of $\alpha$. They satisfy the following properties:

1. $\alpha_{n}=(-1)^{n}\left(q_{n} \alpha-p_{n}\right)$

2. $\alpha_{n}=\left\|q_{n} \alpha\right\|$, for $n \geq 1$

3. $1 /\left(q_{n+1}+q_{n}\right)<\alpha_{n}<1 / q_{n+1}$ for $n \geq 0$.

4. $\alpha_{n+2}<\frac{1}{2} \alpha_{n}, q_{n+2} \geq 2 q_{n}$, for $n \geq-1$

We recall that $D C\left(C_{d}, \beta\right)$ denotes the set of Diophantine numbers of constants $\beta$ and $C_{d}$. One of the following relations characterizes $D C\left(C_{d}, \beta\right)$ :

1. $\left|\alpha-p_{n} / q_{n}\right|>C_{d} / q_{n}^{2+\beta}$ for any $n \geq 0$

2. $a_{n+1}<\frac{1}{C_{d}} q_{n}^{\beta}$ for any $n \geq 0$

3. $q_{n+1}<\frac{1}{C_{d}} q_{n}^{1+\beta}$ for any $n \geq 0$

4. $\alpha_{n+1}>C_{d} \alpha_{n}^{1+\beta}$ for any $n \geq 0$

In all the paper, we denote $C_{d}^{\prime}=1 / C_{d}$.

- Let $m_{n}(x)=f^{q_{n}}(x)-x, n \geq 1, x \in \mathbb{T}^{1}$, let $M_{n}=\sup _{x \in \mathbb{T}^{1}}\left|f^{q_{n}}(x)-x\right|$ and $m_{n}=\inf _{x \in \mathbb{T}^{1}}\left|f^{q_{n}}(x)-x\right|$.

- For any $\phi, \psi \in C^{\gamma}\left(\mathbb{T}^{1}\right)$, we have:

$$
\begin{gathered}
|\phi \psi|_{\gamma} \leq\|\phi\|_{0}|\psi|_{\gamma}+|\phi|_{\gamma}\|\psi\|_{0} \\
\|\phi \psi\|_{\gamma} \leq\|\phi\|_{0}|\psi|_{\gamma}+\|\phi\|_{\gamma}\|\psi\|_{0}
\end{gathered}
$$

- For any real numbers $a$ and $b, a \vee b$ denotes $\max (a, b)$.

In the rest of the paper, for any integer $i, C_{i}^{f}$ denotes a constant depending only on $W(f)$ and $|S f|_{0}$ (i.e. $C_{i}^{f}$ is a numerical function of these variables). $C_{i}^{f, k}$ denotes a constant depending only on $k, W(f),|S f|_{0}$ and $|S f|_{k-3}$. $C_{i}$ denotes a constant that might depend on $k, W(f),|S f|_{0},|S f|_{k-3}$ and also $\beta$ and $C_{d}$. 


\section{$3 C^{1}$ estimations: constant type}

\subsection{A 2-parameters family of homographies}

In this subsection, we show the existence of a lower bound on the norm of the conjugacy in function of $C_{d}$ in the particular case of a 2-parameters family of homographies. We also establish an upper bound on the $C^{1}$ norm of the conjugacy for this family. These bounds are similar to what is given by the local KAM theory. However, these bounds are very specific to this setting. Our general bounds given in theorems 1.2 and 1.3 are much larger.

Proposition 3.1. Let $f:\{z \in \mathbb{C} /|z|=1\} \rightarrow\{z \in \mathbb{C} /|z|=1\}$ defined by $f(z)=h^{-1} R_{\theta} h(z)$, with $R_{\theta}(z)=e^{i \theta} z$ and $h$ is a homography defined by:

$$
h(z)=\frac{z-a}{a z-1}
$$

Let $2>a>1$, let $C_{d}$ such that $C_{d}^{-1} \geq 6$ is a positive integer; and $0<\theta=2 \pi C_{d} \leq$ $\pi / 3$, (therefore, $\theta /(2 \pi)=\left[0, C_{d}^{-1}, 1\right]$ is of constant type $\left.C_{d}\right)$. Let $\tilde{f}: \mathbb{T}^{1} \rightarrow \mathbb{T}^{1}$ the circle diffeomorphism induced by $f$ and $\tilde{h}$ the conjugacy induced by $h$. We have the following estimation:

$$
\frac{2}{\pi} C_{15}\left(|D \tilde{f}(0)|,\left|D^{2} \tilde{f}(0)\right|\right) / C_{d} \leq|D \tilde{h}|_{0} \leq 9 C_{15}\left(|D \tilde{f}(0)|,\left|D^{2} \tilde{f}(0)\right|\right) / C_{d}
$$

Proof. For any $\phi \in \mathbb{R} / \mathbb{Z}$, we can write $h\left(e^{i \phi}\right)=e^{i \tilde{h}(\phi)}$. By differentiating this expression, we have:

$$
D \tilde{h}(\phi)=e^{i \phi} \frac{D h\left(e^{i \phi}\right)}{h\left(e^{i \phi}\right)}
$$

and

$$
\operatorname{Dh}(z)=\frac{(a-1)(a+1)}{(a z-1)^{2}}
$$

Therefore

$$
D \tilde{h}(\phi)=e^{i \phi} \frac{a^{2}-1}{\left(a e^{i \phi}-1\right)\left(e^{i \phi}-a\right)}
$$

$|D \tilde{h}(\phi)|$ reaches its maximum for $\phi=0$, and $|D \tilde{h}|_{0}=\frac{a+1}{a-1}$.

Moreover, we have:

$$
\frac{D^{2} \tilde{f}(\phi)}{D \tilde{f}(\phi)}=i+i e^{i \phi} \frac{D^{2} f\left(e^{i \phi}\right)}{D f\left(e^{i \phi}\right)}-i e^{i \phi} \frac{D f\left(e^{i \phi}\right)}{f\left(e^{i \phi}\right)}
$$

Since $\frac{D^{2} \tilde{f}(\phi)}{D \tilde{f}(\phi)} \in \mathbb{R}$ and $D \tilde{f}(\phi) \in \mathbb{R}$, we have:

$$
\left|\frac{D^{2} f(1)}{D f(1)}\right|=\left(\left(\frac{D^{2} \tilde{f}(0)}{D \tilde{f}(0)}\right)^{2}+(D \tilde{f}(0)-1)^{2}\right)^{1 / 2}=8 C_{15}\left(|D \tilde{f}(0)|,\left|D^{2} \tilde{f}(0)\right|\right)
$$

Therefore, in order to get the proposition, it suffices to show:

$$
\frac{1}{4 \pi C_{d}}\left|\frac{D^{2} f(1)}{D f(1)}\right| \leq|D h|_{0} \leq \frac{9}{8 C_{d}}\left|\frac{D^{2} f(1)}{D f(1)}\right|
$$


Let us write

$$
f(z)=\frac{\left(e^{i \theta}-a^{2}\right) z-a\left(e^{i \theta}-1\right)}{a\left(e^{i \theta}-1\right) z-\left(a^{2} e^{i \theta}-1\right)}=\frac{b z-c}{c z+d}
$$

We have

$$
D f(z)=\frac{d b+c^{2}}{(c z+d)^{2}}
$$

and

$$
D^{2} f(z)=-2 \frac{D f(z)}{z+d / c}
$$

Moreover,

$$
D f(1)=\frac{(1+a)^{2} e^{i \theta}}{\left(a e^{i \theta}+1\right)^{2}}
$$

and

$$
D^{2} f(1)=-2 D f(1) \frac{a\left(e^{i \theta}-1\right)}{(1-a)\left(1+a e^{i \theta}\right)}
$$

We have

$$
\left|\frac{D^{2} f(1)}{D f(1)}\right|=2\left|\frac{a}{1+a e^{i \theta}}\right| \frac{\left|e^{i \theta}-1\right|}{a-1}
$$

Since $\left|e^{i \theta}-1\right| \geq \sin \theta \geq \frac{2}{\pi} \theta$ (because $0 \leq \theta \leq \pi / 2$ ), then:

$$
\left|\frac{D^{2} f(1)}{D f(1)}\right| \geq \frac{4}{\pi} \frac{a}{1+a} \frac{\theta}{a-1}
$$

Therefore,

$$
\left|\frac{D^{2} f(1)}{D f(1)}\right| \geq \frac{4}{\pi} \frac{a}{(1+a)^{2}}|D h|_{0} \theta
$$

i.e.

$$
\frac{9 \pi}{4 \theta}\left|\frac{D^{2} f(1)}{D f(1)}\right| \geq|D h|_{0}
$$

Hence the first part of the inequality.

On the other hand, since $\theta \leq \pi / 3$, then $\left|1+a e^{i \theta}\right| \geq 1+a \cos \theta \geq 1+a / 2 \geq \frac{1}{2}(a+1)$.

Furthermore, $\left|e^{i \theta}-1\right|^{2}=2-2 \cos \theta=2-2\left(\cos ^{2} \theta / 2-\sin ^{2} \theta / 2\right)=4 \sin ^{2} \theta / 2 \leq \theta^{2}$. Therefore,

$$
\left|\frac{D^{2} f(1)}{D f(1)}\right| \leq \frac{\theta}{a-1} \frac{4 a}{a+1} \leq \frac{4 \theta}{a-1}=\frac{4 \theta|D h|_{0}}{a+1}
$$

i.e.

$$
\frac{1}{2}\left|\frac{D^{2} f(1)}{\theta D f(1)}\right| \leq|D h|_{0}
$$

Hence the second part of the inequality. 


\subsection{Proof of theorem 1.2}

The proof of theorem 1.2 is divided in three steps. The first step is based on the improved Denjoy inequality, which estimates the $C^{0}$-norm of $\log D f^{q_{l}}$. In the second step, we extend this estimation to $\log D f^{N}$ for any integer $N$. To do this, following Denjoy and Herman, we write $N=\sum_{s=0}^{S} b_{s} q_{s}$, with $b_{s}$ integers satisfying $0 \leq b_{s} \leq q_{s+1} / q_{s}$ and we apply the chain rule. In the third step, we derive a $C^{0}$-estimation of the derivative $D h$ of the conjugacy $h$.

The first step is based on the Denjoy inequality:

Proposition 3.2. Let $f \in \operatorname{Diff}_{+}^{3}\left(\mathbb{T}^{1}\right)$ and $x \in \mathbb{T}^{1}$. We have:

$$
\left|\log D f^{q_{l}}(x)\right| \leq W(f)
$$

Proposition 3.2 is used to obtain an improved version of Denjoy inequality [14, p.342]:

Lemma 3.3. Let $f \in \operatorname{Diff}_{+}^{3}\left(\mathbb{T}^{1}\right)$. We have:

$$
\begin{aligned}
& \left|\log D f^{q_{l}}\right|_{0} \leq C_{16}^{f} M_{l}^{1 / 2} \\
& \left|D f^{q_{l}}-1\right|_{0} \leq C_{17}^{f} M_{l}^{1 / 2}
\end{aligned}
$$

Moreover, we can take:

$$
C_{16}^{f}=2 \sqrt{2}\left(2 e^{W(f)}+1\right) e^{W(f)}\left(|S f|_{0}\right)^{1 / 2}
$$

and

$$
C_{17}^{f}=6 \sqrt{2} e^{3 W(f)}|S f|_{0}^{1 / 2}
$$

In the second step, we estimate $D \log D f^{N}$ independently of $N$. This step is based on the following lemma:

Lemma 3.4. Let $f \in \operatorname{Diff} f_{+}^{3}\left(\mathbb{T}^{1}\right)$ and $M_{l}=\sup _{x \in \mathbb{T}^{1}}\left|f^{q_{l}}(x)-x\right|$. We have:

$$
\sum_{l \geq 0} \sqrt{M_{l}} \leq \frac{1}{\sqrt{C_{19}^{f}}-C_{19}^{f}}
$$

with

$$
C_{19}^{f}=\frac{1}{\sqrt{1+e^{-C_{22}^{f}}}}
$$

and:

$$
C_{22}^{f}=6 \sqrt{2} e^{2 W(f)}\left(|S f|_{0}^{1 / 2} \vee 1\right)
$$

Proof. To obtain this lemma, we need the claim: 
Claim 3.5. Let $f \in$ Diff? ${ }_{+}^{2}\left(\mathbb{T}^{1}\right)$ of rotation number $\alpha$, and let $p_{n} / q_{n}$ be the convergents of $\alpha$. Then for all $x \in \mathbb{T}^{1}$, we have:

$$
\left[x, f^{2 q_{l+2}}(x)\right] \subset\left[x, f^{q_{l}}(x)\right]
$$

Proof. By topological conjugation, it suffices to examine the case of a rotation of angle $\alpha$. It is also sufficient to take $x=0$.

By absurd, if the lemma was false, then we would have the following cyclic order on $\mathbb{T}^{1}:-q_{l+2} \alpha \leq\left(q_{l+2}-q_{l}\right) \alpha \leq 0 \leq\left(q_{l}-q_{l+2}\right) \alpha \leq q_{l+2} \alpha$. In particular, $\left(q_{l+2}-q_{l}\right) \alpha$ would be closer to 0 than $q_{l+2} \alpha$, which would contradict the fact that

$\left\|q_{l+2} \alpha\right\|=\inf \left\{\|q \alpha\| / 0<q \leq q_{l+2}\right\}$.

For any interval $I$ of the circle, if $|I|$ denotes the length of $I$, lemma 3.3 implies the estimation:

$$
\frac{\left|f^{q_{l+2}}(I)\right|}{|I|} \geq e^{-C_{22}^{f} M_{l+2}^{1 / 2}}
$$

Let $x \in \mathbb{T}^{1}$ such that $M_{l+2}=f^{q_{l+2}}(x)-x$ and let $I=\left[x, f^{q_{l+2}}(x)\right]$. The former estimation implies

$$
\left|f^{2 q_{l+2}}(x)-f^{q_{l+2}}(x)\right| \geq e^{-C_{22}^{f} M_{l+2}^{1 / 2}} M_{l+2}
$$

By applying claim 3.5, and since $M_{n} \leq 1$, we obtain:

$$
M_{n+2}+e^{-C_{22}^{f}} M_{n+2} \leq M_{n+2}+e^{-C_{22}^{f} M_{n+2}^{1 / 2}} M_{n+2} \leq M_{n}
$$

Therefore, for any $l \geq 0$,

$$
M_{l} \leq\left(C_{19}^{f}\right)^{l-1}
$$

with

$$
C_{19}^{f}=\frac{1}{\sqrt{1+e^{-C_{22}^{f}}}}
$$

Estimation (12) above gives:

$$
\sum_{l \geq 0} \sqrt{M_{l}} \leq \frac{1}{\sqrt{C_{19}^{f}}} \frac{1}{1-\sqrt{C_{19}^{f}}} \leq \frac{1}{\sqrt{C_{19}^{f}}-C_{19}^{f}}
$$

Hence lemma 3.4.

Now, let $N$ be an integer. Following Denjoy, since $\alpha$ is of constant type, we can write $N=\sum_{l=0}^{s} b_{l} q_{l}$, with $b_{l}$ integers satisfying $0 \leq b_{l} \leq q_{l+1} / q_{l} \leq C_{d}^{-1}$. By the chain rule and by lemma 3.3 , since for all $y \in \mathbb{T}^{1}, D f^{N}(y)>0$, then :

$$
\begin{gathered}
\left|\log D\left(f^{N}\right)(y)\right|=\left|\log D\left(f^{\sum_{l=0}^{s} b_{l} q_{l}}\right)(y)\right|=\left|\sum_{l=0}^{s} \sum_{i=0}^{b_{s}} \log D f^{q_{l}} \circ f^{i q_{l}}(y)\right| \\
\leq\left.\sup _{0 \leq l \leq s} b_{l} \sum_{l=0}^{s}|\log | D\left(f^{q_{l}}\right)\right|_{0} \mid \leq C_{d}^{-1} C_{22}^{f} \sum_{l \geq 0} M_{l}^{1 / 2}
\end{gathered}
$$


By taking the upper bound on $y \in \mathbb{T}^{1}$ and $N \geq 0$, we obtain an estimation of $\sup _{N \geq 0}\left|\log D\left(f^{N}\right)\right|$.

We turn to the third step: we relate the norms of $D h$ and $D f^{N}$. By [14], $h$ is $C^{1}$ and conjugates $f$ to a rotation. Therefore, we have:

$$
\log D h-\log D h \circ f=\log D f
$$

hence, for all $n$ integer:

$$
\log D h-\log D h \circ f^{n}=\log D\left(f^{n}\right)
$$

Since there is a point $z$ in the circle such that $D h(z)=1$, we then have:

$$
\left|\log D h \circ f^{n}(z)\right|=\left|\log D\left(f^{n}\right)(z)\right| \leq \sup _{i \geq 0}\left|\log D\left(f^{i}\right)\right|_{0}
$$

Moreover, since $\left(f^{n}(z)\right)_{n \geq 0}$ is dense in the circle, and since $D h$ is continuous, then we obtain:

$$
|\log D h|_{0} \leq \sup _{i \geq 0}\left|\log D\left(f^{i}\right)\right|_{0}
$$

We conclude:

$$
|D h|_{0} \leq \exp \left(C_{d}^{-1} C_{22}^{f} \sqrt{e^{C_{22}^{f} \max \left(M_{0}^{1 / 2}, M_{1}^{1 / 2}\right)}+1}\left(\sqrt{M_{0}}+\sqrt{M_{1}}\right)\right)
$$

Finally, since $\max \left(M_{0}^{1 / 2}, M_{1}^{1 / 2}\right) \leq 1$, we obtain:

$$
|D h|_{0} \leq \exp \left(C_{1}^{f} / C_{d}\right)
$$

where $C_{1}^{f}=2 C_{22}^{f} \sqrt{e^{C_{22}^{f}}+1}$. We recall that:

$$
C_{22}^{f}=6 \sqrt{2} e^{2 W(f)}\left(|S f|_{0}^{1 / 2} \vee 1\right)
$$

Hence the theorem.

Corollary 3.6. Since $\frac{1}{\min _{\mathbb{T}^{1}} D h} \leq \exp \left(\sup _{i \geq 0}\left|\log D\left(f^{i}\right)\right|_{0}\right)$, the proof above also provides an estimation on $\frac{1}{\min _{\mathrm{T}^{1}} D h}$ :

$$
\frac{1}{\min _{\mathbb{T}^{1}} D h} \leq \exp \left(C_{1}^{f} / C_{d}\right)
$$

\section{$4 C^{1}$ estimations: non-constant type}

We have $\max _{n \geq 0}\left|D f^{n}\right|_{0} \leq \max _{n \geq 0} M_{n} / m_{n}$, by [14, p. 348]. Therefore, in order to prove theorem 1.3, we can estimate $M_{n} / m_{n}$. To that end, we proceed in two steps: first, we establish some preliminary results. The most important result is corollary 4.6, which gives an estimation of $M_{n+1} / M_{n}$ in function of $M_{n}, \alpha_{n+1} / \alpha_{n}$ and a constant $C_{28}^{f, k}$. This estimation is already given in [14, p. 345], but we still recall the steps to reach it, because we need to estimate the constant $C_{28}^{f, k}$ in function of $k, W(f),|S f|_{0}$ and $|S f|_{k-3}$.

In the second step, we establish an estimation of the $C^{1}$-conjugacy, based on a modification of the proof given in [14]. The main idea is to establish an alternative between 
two possible situations for the sequences $M_{n}$ and $\alpha_{n}$ : the "favorable" situation $\left(R_{n}\right)$ and the "unfavorable" situation $\left(R_{n}^{\prime}\right)$ (proposition 4.10). The "unfavorable" situation only occurs a finite number of times, due to the Diophantine condition on $\alpha$ (propositions 4.12 and 4.14).

In the "favorable" situation $\left(R_{n}\right)$, we can estimate $M_{n+1} / \alpha_{n+1}$ in function of $M_{n} / \alpha_{n}$ (see estimation (26)) and likewise, we can estimate $\alpha_{n+1} / m_{n+1}$ in function of $\alpha_{n} / m_{n}$. Therefore, we can estimate $M_{n} / m_{n}$ in function of $M_{n_{4}} / m_{n_{4}}$, where $n_{4}$ is the integer such that for any $n \geq n_{4}$, the favorable case occurs (see proposition 4.19). We relate

$M_{n_{4}} / m_{n_{4}}$ to $\left.D f\right|_{0} ^{\frac{2}{\alpha_{n_{4}}}}$ (proposition 4.17), and we compute a bound on $\alpha_{n_{4}}$ (proposition 4.15). Yoccoz's proof needs to be modified because in its original version, it does not allow to compute a bound on $\alpha_{n_{4}}$.

\subsection{Preliminary results}

First, we recall the following lemmas, which are in [14] (lemmas 3,4 and 5):

Lemma 4.1. For $l \geq 1$ and $x \in \mathbb{T}^{1}$, we have:

$$
\sum_{i=0}^{q_{n+1}-1}\left(D f^{i}(x)\right)^{l} \leq C_{23}^{f} \frac{M_{n}^{l-1}}{m_{n}(x)^{l}}
$$

with $C_{23}^{f}(l)=e^{l W(f)}$.

Remark 4.2. This lemma is obtained by applying Denjoy inequality.

Lemma 4.3. Let $f \in \operatorname{Diff}_{+}^{k}\left(\mathbb{T}^{1}\right), k \geq 3$. For any $x \in \mathbb{T}^{1}$, any $n \in \mathbb{N}$, any $0 \leq p \leq q_{n+1}$, we have:

$$
\begin{gathered}
\left|S f^{p}\right|_{0} \leq C_{24}^{f} \frac{M_{n}}{m_{n}^{2}} \\
\left|S f^{p}(x)\right| \leq C_{24}^{f} \frac{M_{n}}{m_{n}(x)^{2}} \\
\left|D \log D f^{p}\right|_{0} \leq C_{25}^{f} \frac{M_{n}^{1 / 2}}{m_{n}} \\
\left|D \log D f^{p}(x)\right| \leq C_{26}^{f} \frac{M_{n}^{1 / 2}}{m_{n}(x)}
\end{gathered}
$$

with:

- $C_{24}^{f}=|S f|_{0} e^{2 W(f)}$

- $C_{25}^{f}=\sqrt{2|S f|_{0}} e^{W(f)}$

- $C_{26}^{f}=9 \sqrt{2|S f|_{0}} e^{4 W(f)}$ 
Lemma 4.4. For $1 \leq r \leq k-1, n \geq 0,0 \leq p \leq q_{n+1}, x \in \mathbb{T}^{1}$, we have:

$$
\left|D^{r} \log D f^{p}(x)\right| \leq C_{27}^{f}(r)\left[\frac{M_{n}^{1 / 2}}{m_{n}(x)}\right]^{r}
$$

with

$$
C_{27}^{f}(1)=C_{26}^{f}, C_{27}^{f}(2)=82|S f|_{0} e^{8 W(f)}
$$

and, for $r \geq 3$ :

$$
C_{27}^{f}(r)=\left[82(2 r)^{2 r}\left(1 \vee|S f|_{r-2}\right)^{2} e^{(r+8) W(f)}\right]^{r !}
$$

In particular,

$$
C_{27}^{f, k}:=C_{27}^{f}(k-1) \leq\left[100(2 k-2)^{2 k-2}\left(1 \vee|S f|_{k-3}\right)^{2} e^{(k+7) W(f)}\right]^{(k-1) !}
$$

Proof of lemma 4.4. The proof follows the line of [14], lemma 5: see appendix 6.1.

The important preliminary result, corollary 4.6 , is obtained from the following proposition. It is obtained by computing the constants in proposition 2 of [14]:

Proposition 4.5. Let

$$
C_{28}^{f, k}=(k+3)^{(k+3) !} e^{(k+2) ! W(f)}\left(\max \left(1,|S f|_{k-3}\right)\right)^{k !}
$$

For any $x \in \mathbb{T}^{1}$, we have:

$$
\left|m_{n+1}(x)-\frac{\alpha_{n+1}}{\alpha_{n}} m_{n}(x)\right| \leq C_{28}^{f, k}\left[M_{n}^{(k-1) / 2} m_{n}(x)+M_{n}^{1 / 2} m_{n+1}(x)\right]
$$

Corollary 4.6.

$$
\begin{gathered}
M_{n+1} \leq M_{n} \frac{\frac{\alpha_{n+1}}{\alpha_{n}}+C_{28}^{f, k} M_{n}^{(k-1) / 2}}{1-C_{28}^{f, k} M_{n}^{1 / 2}} \\
m_{n+1} \geq m_{n} \frac{\frac{\alpha_{n+1}}{\alpha_{n}}-C_{28}^{f, k} M_{n}^{(k-1) / 2}}{1+C_{28}^{f, k} M_{n}^{1 / 2}}
\end{gathered}
$$

The proof of proposition 4.5 combines the following three lemmas [14, pp. 343344] (lemmas 6, 7 and 8):

Lemma 4.7. For any $x \in \mathbb{T}^{1}$, there exists $y \in\left[x, f^{q_{n}}(x)\right], z \in\left[f^{q_{n+1}}(x), x\right]$ such that

$$
m_{n+1}(y)=\frac{\alpha_{n+1}}{\alpha_{n}} m_{n}(z)
$$

Lemma 4.8. Suppose that $m_{n+1}$ is monotonous on an interval $I_{z}=\left(z, f^{q}(z)\right), z \in \mathbb{T}^{1}$. Then, for any $x \in \mathbb{T}^{1}$, for any $y \in I_{x}\left(I_{x}=\left(x, f^{q}(x)\right)\right)$, we have:

$$
\left|\frac{m_{n+1}(y)}{m_{n+1}(x)}-1\right| \leq C_{29}^{f, k} M_{n}^{1 / 2}
$$

with

$$
C_{29}^{f, k}=2^{9}(k+2) e^{(11+k / 2) W(f)}\left(C_{17}^{f}\right)^{2} C_{26}^{f}
$$


Lemma 4.9. If $m_{n+1}$ is not monotonous on any interval of the form $I_{z}=\left(z, f^{q}(z)\right)$, $z \in \mathbb{T}^{1}$, then for any $x \in \mathbb{T}^{1}, y \in I_{x}$, we have:

$$
\left|m_{n+1}(y)-m_{n+1}(x)\right| \leq C_{30}^{f, k} M_{n}^{(k-1) / 2} m_{n}(x)
$$

with

$$
C_{30}^{f, k}=\left(C_{27}^{f}(k-1)\right) e^{W(f)}\left(e^{(k / 2+2) W(f)}\left(1+e^{W(f)}\right)^{2} \frac{e^{(k / 2+2) W(f)}-1}{e^{W(f)}-1}\right)^{k-1}
$$

Proof of proposition 4.5. Let us recall the proof of proposition 4.5 from these three lemmas. (see [14, p.344]). Let $x \in \mathbb{T}^{1}$ and $y \in I_{x}, z \in\left[f^{q_{n+1}}(x), x\right]$ the points given by lemma 4.7. By combining lemmas 4.8 and 4.9, we obtain:

$$
\left|m_{n+1}(y)-m_{n+1}(x)\right| \leq\left(\max \left(C_{29}^{f, k}, C_{30}^{f, k}\right)\right)\left(M_{n}^{1 / 2} m_{n+1}(x)+M_{n}^{(k-1) / 2} m_{n}(x)\right)
$$

Moreover, by lemma 3.3, we have:

$$
\left|m_{n}(z)-m_{n}(x)\right| \leq C_{17}^{f} M_{n}^{1 / 2}|z-x| \leq C_{17}^{f} M_{n}^{1 / 2} m_{n+1}(x)
$$

By applying lemma 4.7, and since $\alpha_{n+1} / \alpha_{n} \leq 1$, we get:

$$
\begin{gathered}
\left|m_{n+1}(x)-\frac{\alpha_{n+1}}{\alpha_{n}} m_{n}(x)\right| \leq\left|m_{n+1}(x)-\frac{\alpha_{n+1}}{\alpha_{n}} m_{n}(z)\right|+\frac{\alpha_{n+1}}{\alpha_{n}}\left|m_{n}(z)-m_{n}(x)\right| \\
\left|m_{n+1}(x)-\frac{\alpha_{n+1}}{\alpha_{n}} m_{n}(x)\right| \leq\left|m_{n+1}(y)-m_{n+1}(x)\right|+\left|m_{n}(z)-m_{n}(x)\right|
\end{gathered}
$$

Therefore, we have:

$$
\left|m_{n+1}(x)-\frac{\alpha_{n+1}}{\alpha_{n}} m_{n}(x)\right| \leq C_{31}^{f, k}\left(M_{n}^{1 / 2} m_{n+1}(x)+M_{n}^{(k-1) / 2} m_{n}(x)\right)
$$

with $C_{31}^{f, k}=\max \left(C_{29}^{f, k}, C_{30}^{f, k}\right)+C_{17}^{f}$.

Finally, let us estimate $C_{31}^{f, k}$. Since $k \geq 3$, then:

$[4(k / 2+1)(200 k)]^{2} \leq(k+3)^{(k+3)(k+2) k} / 2$ and therefore,

$$
2^{2(k-1)}(k / 2+1)^{k-1}(200 k)^{2(k+1)(k-1) !} \leq(k+3)^{(k+3) !} / 2
$$

Therefore, we have:

$$
C_{30}^{f, k}+C_{17}^{f} \leq(k+3)^{(k+3) !} e^{(k+2) ! W(f)}\left(\max \left(1,|S f|_{k-3}\right)\right)^{k !}
$$

Since $k \geq 3$, we also have:

$$
C_{29}^{f, k}+C_{17}^{f} \leq(k+3)^{(k+3) !} e^{(k+2) ! W(f)}\left(\max \left(1,|S f|_{k-3}\right)\right)^{k !}
$$

Therefore, $C_{31}^{f, k} \leq C_{28}^{f, k}=(k+3)^{(k+3) !} e^{(k+2) ! W(f)}\left(\max \left(1,|S f|_{k-3}\right)\right)^{k !}$. Hence proposition 4.5. 


\subsection{Estimation of the $C^{1}$-conjugacy. Proof of estimation (1).}

We choose an integer $n_{1}$ such that for any $n \geq n_{1}$, we have:

$$
C_{28}^{f, k} M_{n}^{1 / 2} \leq C_{28}^{f, k}\left(C_{19}^{f}\right)^{\frac{n-1}{2}}<1 / 2
$$

We take:

$$
n_{1}=\left\lceil\frac{-\log \left(2 C_{28}^{f, k} /\left(C_{19}^{f}\right)^{1 / 2}\right)}{\log \left(\left(C_{19}^{f}\right)^{1 / 2}\right)}\right\rceil
$$

We choose a parameter $\theta$ such that $(k+1) / 2-\theta>(1+\beta+\theta)(1+\theta)$ (for the interpretation of this parameter $\theta$, see the remark after proposition 4.10). We take:

$$
\theta=\min \left(1 / 2,\left(\frac{3+\beta}{4}\right)\left(-1+\left(1+\frac{2(k-2 \beta-1)}{(3+\beta)^{2}}\right)^{1 / 2}\right)\right)
$$

(in the proof of estimation (2), we take $\theta=1 / 2$ instead).

We recall that for $x \geq 0,1+x \leq e^{x}$ and for $0 \leq x \leq 1 / 2, \log (1 /(1-x)) \leq$ $x /(1-x) \leq 2 x$. We apply estimation (18), we use the definition of $n_{1}$ and the fact that $\theta \leq 1 / 2$. We get:

$$
\begin{gathered}
\prod_{n=n_{1}}^{+\infty}\left(1+M_{n}^{\theta}\right) \leq \exp \left(\sum_{n=n_{1}}^{+\infty} M_{n}^{\theta}\right) \leq \exp \left(\frac{1}{2 C_{28}^{f, k}\left(1-\left(C_{19}^{f}\right)^{\theta}\right)}\right) \\
\prod_{n=n_{1}}^{+\infty}\left(\frac{1}{1-C_{28}^{f, k} M_{n}^{1 / 2}}\right) \leq \exp \left(\sum_{n=n_{1}}^{+\infty} 2 C_{28}^{f, k} M_{n}^{1 / 2}\right) \leq \exp \left(\frac{1}{1-\left(C_{19}^{f}\right)^{1 / 2}}\right)
\end{gathered}
$$

Therefore,

$$
\prod_{n=n_{1}}^{+\infty}\left(\frac{1+M_{n}^{\theta}}{1-C_{28}^{f, k} M_{n}^{1 / 2}}\right) \leq \exp \left(\frac{2}{1-\left(C_{19}^{f}\right)^{\theta}}\right)=C_{32}
$$

Let:

$$
C_{33}=\max \left(\left(4 C_{28}^{f, k}\right)^{\frac{1}{(1+\beta+\theta)(1+\theta)-1}}, C_{32}\right)
$$

For any

$$
n \geq \frac{-\log \left(2\left(C_{33}\right)^{2}\right)}{\log C_{19}^{f}}+1=C_{34}
$$

we have:

$$
M_{n} \leq\left(C_{19}^{f}\right)^{n-1} \leq \frac{1}{2 C_{33}^{2}}
$$

We use this estimation in the second step of the proof, to which we come now:

Let 


$$
n_{2}=\max \left(n_{1}, \tilde{n}_{2}\right)
$$

where $\tilde{n}_{2}$ is the integer defined by

$$
C_{34}+\frac{4}{\log 2} \log \left(1 / C_{d}\right)+1 \leq \tilde{n}_{2}<C_{34}+\frac{4}{\log 2} \log \left(1 / C_{d}\right)+2
$$

Having defined the integer $n_{2}$, we can present the alternative between the "favorable" case $\left(R_{n}\right)$ and the "unfavorable" case $\left(R_{n}^{\prime}\right)$.

Proposition 4.10. Let $a_{n_{2}}=1 /\left(\left(C_{33}\right)^{2}\right)$. Let $1 \geq \eta_{n} \geq 0$ be a sequence such that $\alpha_{n}=\alpha_{n+1}^{1-\eta_{n}}$. For any $n \geq n_{2}$, we can define a sequence $a_{n}, 1 /\left(\left(C_{33}\right)^{2}\right) \leq a_{n} \leq 1 / C_{33}$ and a sequence $\rho_{n}<1$ such that $M_{n}=a_{n} \alpha_{n}^{\rho_{n}}$. The sequence $a_{n}$ is defined by:

if

$$
\left(R_{n}\right) \quad C_{28}^{f, k} M_{n}^{(k+1) / 2-\theta} \leq M_{n} \frac{\alpha_{n+1}}{\alpha_{n}} \text { then } a_{n+1}=a_{n} \frac{1+M_{n}^{\theta}}{1-C_{28}^{f, k} M_{n}^{1 / 2}}
$$

and if

$$
\left(R_{n}^{\prime}\right) \quad C_{28}^{f, k} M_{n}^{(k+1) / 2-\theta}>M_{n} \frac{\alpha_{n+1}}{\alpha_{n}} \text { then } a_{n+1}=a_{n}
$$

Moreover, if $\left(R_{n}\right)$ holds, then $\rho_{n+1} \geq \rho_{n}+\eta_{n}\left(1-\rho_{n}\right)$;

and if $\left(R_{n}^{\prime}\right)$ holds, then $\rho_{n+1} \geq((k+1) / 2-\theta)\left(1-\eta_{n}\right) \rho_{n}$. In particular, the sequence $\left(\rho_{n}\right)_{n \geq n_{2}}$ is increasing.

The threshold between the alternatives $\left(R_{n}\right)$ and $\left(R_{n}^{\prime}\right)$ is controlled with a parameter $\theta$, which could be freely chosen such that $\theta>0$ and $(k+1) / 2-\theta \geq(1+\beta+\theta)(1+\theta)$. When $\theta$ increases, the number $n_{3}$ of occurrences of $\left(R_{n}^{\prime}\right)$ increases. When $n_{3}$ increases, all other quantities being equal, the bound on the norm of the conjugacy increases. Moreover, if $\theta$ gets too large, we can no longer show that $n_{3}$ is finite (see proposition 4.14), and therefore, we can no longer estimate the norm of the conjugacy.

On the other hand, when $\theta$ is smaller, $C_{32}$ increases. It increases the number $n_{2}$ above which we consider the alternatives $\left(R_{n}\right)$ and $\left(R_{n}^{\prime}\right) . C_{35}$ increases too (see proposition 4.19). When $C_{32}$ and $C_{35}$ increase, all other quantities being equal, the bound on the norm of the conjugacy increases. Moreover, when $\theta \rightarrow 0, C_{32} \rightarrow+\infty$, which makes this bound on the conjugacy diverge.

Thus, the variation of $\theta$ has contradictory influences on the bound of the norm of the conjugacy, and there is a choice of $\theta$ that optimizes this bound. However, in this paper, we do not seek this optimal $\theta$, since it would complicate further the expression of the final estimate. Instead, in estimation (2), we fix $\theta=1 / 2$, which allows simplifying the expression of the estimate. In estimation (3), we take $\theta \rightarrow 0$, which also allows simplifying the estimate.

Proof of proposition 4.10: For any $n \geq n_{2}$, since $n_{2} \geq n_{1}$,

$$
a_{n_{2}}=\frac{1}{C_{33}^{2}} \leq a_{n} \leq a_{n_{2}} \prod_{n=n_{1}}^{+\infty}\left(\frac{1+M_{n}^{\theta}}{1-C_{28}^{f, k} M_{n}^{1 / 2}}\right) \leq \frac{C_{32}}{C_{33}^{2}} \leq \frac{1}{C_{33}}
$$

and since 


$$
\alpha_{n}^{\rho_{n}}>a_{n} \alpha_{n}^{\rho_{n}}=M_{n} \geq \alpha_{n}
$$

then $\rho_{n}<1$.

Second, if $\left(R_{n}\right)$ holds, then by applying corollary 4.6 , we have:

$$
M_{n+1} \leq \frac{1+M_{n}^{\theta}}{1-C_{28}^{f, k} M_{n}^{1 / 2}} M_{n} \frac{\alpha_{n+1}}{\alpha_{n}}
$$

Therefore,

$$
M_{n+1}=a_{n+1} \alpha_{n+1}^{\rho_{n+1}} \leq a_{n+1} \alpha_{n+1} \alpha_{n}^{\rho_{n}-1}=a_{n+1} \alpha_{n+1} \alpha_{n+1}^{\left(1-\eta_{n}\right)\left(\rho_{n}-1\right)}
$$

and then:

$$
\rho_{n+1}-1 \geq\left(1-\eta_{n}\right)\left(\rho_{n}-1\right)
$$

hence the estimation:

$$
\rho_{n+1} \geq \rho_{n}+\eta_{n}\left(1-\rho_{n}\right)
$$

If $\left(R_{n}^{\prime}\right)$ holds, since $C_{28}^{f, k} M_{n}^{1 / 2} \leq 1 / 2$, then by applying corollary 4.6, we obtain:

$$
M_{n+1} \leq 4 C_{28}^{f, k} M_{n}^{(k+1) / 2-\theta}
$$

Moreover, since $a_{n} \leq 1 / C_{33}<1$, then:

$$
a_{n}^{(k+1) / 2-\theta} \leq a_{n}^{(1+\beta+\theta)(1+\theta)}=a_{n} a_{n}^{(1+\beta+\theta)(1+\theta)-1} \leq \frac{a_{n}}{C_{33}^{(1+\beta+\theta)(1+\theta)-1}} \leq \frac{a_{n}}{4 C_{28}^{f, k}}
$$

Therefore, by combining these two estimations, we obtain:

$$
a_{n+1} \alpha_{n+1}^{\rho_{n+1}}=M_{n+1} \leq 4 C_{28}^{f, k} M_{n}^{(k+1) / 2-\theta} \leq 4 C_{28}^{f, k} a_{n}^{(k+1) / 2-\theta} \alpha_{n}^{\rho_{n}((k+1) / 2-\theta)} \leq a_{n} \alpha_{n}^{\rho_{n}((k+1) / 2-\theta)}
$$

Moreover, since $a_{n+1}=a_{n}$, then

$$
1 \leq \alpha_{n+1}^{\left(\rho_{n}((k+1) / 2-\theta)\right)\left(1-\eta_{n}\right)-\rho_{n+1}}
$$

hence the estimation:

$$
\rho_{n+1} \geq\left(\rho_{n}((k+1) / 2-\theta)\right)\left(1-\eta_{n}\right)
$$

The reader can notice that until now, we have not used the Diophantine condition on $\alpha$ yet. Now, we introduce this condition in order to estimate $\rho_{n_{2}}$ from below (proposition 4.11), and in order to determine a bound $\rho$ above which $\left(R_{n}\right)$ always occurs (proposition 4.12). 
Proposition 4.11. If $\beta>0$, we have the estimation:

$$
\rho_{n_{2}} \geq \frac{\log 2}{\left((1+\beta)^{n_{2}+1}-1\right) \log \left(1 / C_{d}\right) / \beta}
$$

If $\beta=0$, we have the estimation:

$$
\rho_{n_{2}} \geq \frac{\log 2}{\left(n_{2}+1\right) \log \left(1 / C_{d}\right)}
$$

Proof. Since $\alpha$ is Diophantine, we have: $\alpha_{n+1} \geq C_{d} \alpha_{n}^{1+\beta}$. Therefore, for $\beta>0$,

$$
\log \left(\frac{1}{\alpha_{n+1}}\right)+\frac{\log \left(1 / C_{d}\right)}{\beta} \leq(1+\beta)\left(\log \left(1 / \alpha_{n}\right)+\frac{\log \left(1 / C_{d}\right)}{\beta}\right)
$$

and since $\alpha_{-1}=1$, then by iteration, for any $n \geq 0$,

$$
\log \left(1 / \alpha_{n}\right) \leq\left((1+\beta)^{n+1}-1\right) \frac{\log \left(1 / C_{d}\right)}{\beta}
$$

If $\beta=0$, we have:

$$
\log \left(1 / \alpha_{n}\right) \leq(n+1) \log \left(1 / C_{d}\right)
$$

Moreover, since $\rho_{n_{2}}=-\log \left(M_{n_{2}} / a_{n_{2}}\right) / \log \left(1 / \alpha_{n_{2}}\right)$ and $M_{n_{2}} / a_{n_{2}} \leq 1 / 2$, then we get proposition 4.11 .

Proposition 4.12. Let $\beta_{1}=\beta+\frac{2 \log \left(1 / C_{d}\right)}{\left(n_{2}-1\right) \log 2}$. If

$$
\rho_{n} \geq \frac{\beta_{1}}{(k-1) / 2-\theta}=\rho
$$

then $\left(R_{n}\right)$ occurs.

Remark 4.13. Note that $\rho<1$, because $(k+1) / 2-\theta \geq(1+\beta+\theta)(1+\theta)$ and $\beta_{1} \leq \beta+1 / 2$.

Proof. Since $\alpha_{n} \leq(1 / 2)^{\frac{n-1}{2}}$, then

$$
0<\frac{\log C_{d}}{\log \alpha_{n}} \leq \frac{-\log C_{d}}{\frac{n-1}{2} \log 2}
$$

Furthermore, since $\alpha_{n+1}=\alpha_{n}^{\frac{1}{1-\eta_{n}}} \geq C_{d} \alpha_{n}^{1+\beta}$, then

$$
\frac{1}{1-\eta_{n}} \log \alpha_{n} \geq \log C_{d}+(1+\beta) \log \alpha_{n}
$$

and since $\log \alpha_{n}<1$ for $n \geq 0$, then by (28),

$$
\frac{1}{1-\eta_{n}}-1 \leq \beta+\frac{\log C_{d}}{\log \alpha_{n}} \leq \beta+\frac{\log \left(1 / C_{d}\right)}{\frac{n-1}{2} \log 2}
$$

Therefore, if estimation (27) holds, then

$$
\left(\frac{k-1}{2}-\theta\right) \rho_{n}+1-\frac{1}{1-\eta_{n}} \geq 0
$$


and therefore,

$$
\left(\frac{1}{\alpha_{n}}\right)^{\left(\frac{k-1}{2}-\theta\right) \rho_{n}+1-\frac{1}{1-\eta_{n}}} \geq 1
$$

Hence

$M_{n} \frac{\alpha_{n+1}}{\alpha_{n}}=a_{n} \alpha_{n}^{\rho_{n}} \frac{\alpha_{n+1}}{\alpha_{n}} \geq a_{n} \alpha_{n}^{\left(\frac{k+1}{2}-\theta\right) \rho_{n}}=M_{n}^{\frac{k+1}{2}-\theta} a_{n}^{1-\left(\frac{k+1}{2}-\theta\right)} \geq M_{n}^{\frac{k+1}{2}-\theta} C_{33}^{\frac{k+1}{2}-\theta-1} \geq M_{n}^{\frac{k+1}{2}-\theta} C_{33}^{(1+\beta+\theta)(1+\theta)-1}$

Therefore,

$$
M_{n} \frac{\alpha_{n+1}}{\alpha_{n}} \geq C_{28}^{f, k} M_{n}^{\frac{k+1}{2}-\theta}
$$

Proposition 4.14. The alternative $\left(R_{n}^{\prime}\right)$ occurs less than $n_{3}$ times, with

$$
n_{3}-n_{2} \leq \max \left(0, \frac{\log \left(\rho / \rho_{n_{2}}\right)}{\log \left(\frac{(k+1) / 2-\theta}{1+\beta_{1}}\right)}\right)
$$

Proof. If $\rho_{n_{2}} \geq \rho$, then $\left(R_{n}^{\prime}\right)$ does not occur for any $n \geq n_{2}$. We suppose $\rho_{n_{2}}<\rho$. For any $n \geq n_{2}$, since

$$
((k+1) / 2-\theta)\left(1-\eta_{n}\right) \geq \frac{(k+1) / 2-\theta}{1+\beta_{1}}
$$

then

$$
\rho_{n} \geq\left(\frac{(k+1) / 2-\theta}{1+\beta_{1}}\right)^{n-n_{2}} \rho_{n_{2}}
$$

Moreover,

$$
\left(\frac{(k+1) / 2-\theta}{1+\beta_{1}}\right)^{n-n_{2}} \rho_{n_{2}} \geq \rho
$$

when

$$
n \geq n_{2}+\frac{\log \left(\rho / \rho_{n_{2}}\right)}{\log \left(\frac{(k+1) / 2-\theta}{1+\beta_{1}}\right)}
$$

The next proposition gives a lower bound on $\alpha_{n_{4}}$, which allows computing a bound on the $C^{1}$-conjugacy.

Proposition 4.15. Let $n_{4} \geq 0$ be the smallest integer such that for any $n \geq n_{4},\left(R_{n}\right)$ occurs. We have:

$$
\alpha_{n_{4}} \geq C_{d}^{\exp \left(\left(n_{3}+1+\rho /(1-\rho)\right)\left(1+\beta_{1}\right)\right)}
$$

Proof. First, we suppose $n_{4} \geq n_{2}+1$ We need the lemma: 
Lemma 4.16. Let $n_{5} \geq n_{2}$ be the smallest integer such that

$$
\sum_{n=n_{2}}^{n_{5}} \eta_{n} \geq n_{3}-n_{2}+\rho /(1-\rho)
$$

$n_{5}$ exists. Moreover, we have $\rho_{n_{5}+1} \geq \rho$. In particular, for this integer $n_{5}$, we have that for any $n \geq n_{5}+1,\left(R_{n}\right)$ occurs.

Proof. First, let us show the existence of $n_{5}$. By absurd, suppose that

$$
\sum_{n=n_{2}}^{+\infty} \eta_{n}<n_{3}-n_{2}+\rho /(1-\rho)
$$

For any $1>x \geq 0$,

$$
\log \left(\frac{1}{1-x}\right) \leq \frac{x}{1-x}
$$

Therefore, for any integer $p \geq n_{2}+1$,

$$
\prod_{n=n_{2}}^{p-1}\left(\frac{1}{1-\eta_{n}}\right) \leq \exp \left(\sum_{n=n_{2}}^{p-1} \frac{\eta_{n}}{1-\eta_{n}}\right)
$$

Moreover, $\frac{1}{1-\eta_{n}} \leq 1+\beta_{1}$ for any $n \geq 1$. Therefore,

$$
\sum_{n=n_{2}}^{p-1} \frac{\eta_{n}}{1-\eta_{n}} \leq\left(n_{3}-n_{2}+\rho /(1-\rho)\right)\left(1+\beta_{1}\right)
$$

Since $\eta_{n} \leq 1$, then $\sum_{n=0}^{n_{2}-1} \eta_{n} \leq n_{2}$. Therefore,

$$
\sum_{n=0}^{p-1} \frac{\eta_{n}}{1-\eta_{n}} \leq\left(n_{3}+\rho /(1-\rho)\right)\left(1+\beta_{1}\right)
$$

Moreover, since $\alpha_{0}=\alpha \geq C_{d}$ then for any $p \geq n_{2}+1$ :

$$
\alpha_{p}=\alpha_{0}^{\prod_{n=0}^{p-1}\left(\frac{1}{1-\eta_{n}}\right)} \geq C_{d}^{\exp \left(\left(n_{3}+\rho /(1-\rho)\right)\left(1+\beta_{1}\right)\right)}
$$

However, since $\alpha_{p} \geq 2 \alpha_{p+2}$, then $\alpha_{p} \rightarrow 0$ when $p \rightarrow+\infty$. Hence the contradiction and the existence of $n_{5}$. Note that $n_{5}+1 \geq n_{4}$.

Second, let us show that $\rho_{n_{5}+1} \geq \rho$. If there is $n_{6} \leq n_{5}$ such that $\rho_{n_{6}} \geq \rho$, then $\rho_{n_{5}+1} \geq \rho$ because the sequence $\rho_{n}$ is increasing. Otherwise, for any $n \leq n_{5}$, we have: $\rho_{n} \leq \rho$.

Let $E_{1}=\left\{n_{5} \geq n \geq n_{2} /\left(R_{n}\right)\right.$ occurs $\}$ and $E_{2}=\left\{n_{5} \geq n \geq n_{2} /\left(R_{n}^{\prime}\right)\right.$ occurs $\}$.

We have:

$$
n_{3}-n_{2}+\frac{\rho}{1-\rho} \leq \sum_{n=n_{2}}^{n_{5}} \eta_{n}=\sum_{n \in E_{1}} \eta_{n}+\sum_{n \in E_{2}} \eta_{n} \leq \sum_{n \in E_{1}} \eta_{n}+n_{3}-n_{2}
$$

Therefore, 


$$
\sum_{n \in E_{1}} \eta_{n} \geq \rho /(1-\rho)
$$

Since $\rho_{n}$ is increasing and $\rho_{n} \leq \rho$, we get:

$$
\begin{gathered}
\rho_{n_{5}+1}=\rho_{n_{2}}+\sum_{n=n_{2}}^{n_{5}} \rho_{n+1}-\rho_{n} \\
\rho_{n_{5}+1} \geq \rho_{n_{2}}+\sum_{n \in E_{1}} \rho_{n+1}-\rho_{n} \geq \rho_{n_{2}}+\sum_{n \in E_{1}}\left(1-\rho_{n}\right) \eta_{n} \geq \rho_{n_{2}}+(1-\rho) \sum_{n \in E_{1}} \eta_{n} \geq \rho
\end{gathered}
$$

Now, let us show proposition 4.15 . Since $\eta_{n} \leq 1$ for any $n$, then we have:

$$
n_{3}-n_{2}+1+\frac{\rho}{1-\rho}>\sum_{n=n_{2}}^{n_{5}} \eta_{n} \geq n_{3}-n_{2}+\frac{\rho}{1-\rho}
$$

Since

$$
n_{3}-n_{2}+\frac{\rho}{1-\rho}+1 \geq \sum_{n=n_{2}}^{n_{5}} \eta_{n} \geq \sum_{n=n_{2}}^{n_{4}-1} \eta_{n}
$$

then by proceeding in the same way as in the first part of the proof of lemma 4.16, we obtain:

$$
\alpha_{n_{4}} \geq C_{d}^{\exp \left(\left(n_{3}+1+\rho /(1-\rho)\right)\left(1+\beta_{1}\right)\right)}
$$

Finally, if $n_{4} \leq n_{2}$, then as in the proof of lemma 4.16,

$$
\alpha_{n_{2}}=\alpha_{0}^{\prod_{n=0}^{n_{2}-1}\left(\frac{1}{1-\eta_{n}}\right)} \geq C_{d}^{\exp \left(n_{2}\left(1+\beta_{1}\right)\right)}
$$

Therefore, the estimation given in proposition 4.15 still holds.

Having bounded $\alpha_{n_{4}}$ from below, we show how this bound is related to $M_{n} / m_{n}$ (and therefore, how this is related to the conjugacy).

Proposition 4.17. Let $n \geq 1, M_{n}=\max _{x \in \mathbb{T}^{1}} m_{n}(x), m_{n}=\min _{x \in \mathbb{T}^{1}} m_{n}(x)$. For any $j \leq n$,

$$
\frac{M_{j}}{m_{j}} \leq 3|D f|_{0}^{\frac{2}{\alpha_{n}}}
$$

Proof. We need the following lemma, which is in [14, p. 339]:

Lemma 4.18. For any $x \in \mathbb{T}^{1}$, let $J_{x}=\left[f^{-q_{n}}(x), f^{q_{n}}(x)\right]$. The intervals $f^{i}\left(J_{x}\right), 0 \leq i<$ $q_{n+1}$ recover $\mathbb{T}^{1}$. 
First, note that since $f(x+1)-f(x)=1$ (in the universal cover), then $|D f|_{0} \geq 1$. Let $x, y \in \mathbb{T}^{1}$ such that $M_{n}=m_{n}(x)$ and $m_{n}=m_{n}(y)$. Let $0 \leq i<q_{n+1}$ such that $x \in f^{i}\left(J_{y}\right)$. Since we have the cyclic order $f^{i-q_{n}}(y) \leq x \leq f^{i+q_{n}}(y)$ then we also have: $f^{i}(y) \leq f^{q_{n}}(x) \leq f^{i+2 q_{n}}(y)$. Therefore, $\left[x, f^{q_{n}}(x)\right] \subset\left[f^{i-q_{n}}(y), f^{i+2 q_{n}}(y)\right]$. This implies:

$$
\begin{gathered}
M_{n} \leq f^{i+2 q_{n}}(y)-f^{i+q_{n}}(y)+f^{i+q_{n}}(y)-f^{i}(y)+f^{i}(y)-f^{i-q_{n}}(y) \\
M_{n} \leq\left(\left|D f^{i+q_{n}}\right|_{0}+\left|D f^{i}\right|_{0}+\left|D f^{i-q_{n}}\right|_{0}\right)\left(f^{q_{n}}(y)-y\right)
\end{gathered}
$$

and therefore,

$$
\frac{M_{n}}{m_{n}} \leq\left(\left|D f^{i+q_{n}}\right|_{0}+\left|D f^{i}\right|_{0}+\left|D f^{i-q_{n}}\right|_{0}\right)
$$

Therefore,

$$
\frac{M_{n}}{m_{n}} \leq 3|D f|_{0}^{q_{n}+q_{n+1}}
$$

Likewise, for any $j \leq n$, we have:

$$
\frac{M_{j}}{m_{j}} \leq 3|D f|_{0}^{q_{n}+q_{n+1}}
$$

Since $q_{n}+q_{n+1} \leq 2 q_{n+1} \leq \frac{2}{\alpha_{n}}$, we obtain proposition 4.17 .

Proposition 4.19. For any $n \geq 1$,

$$
\frac{M_{n}}{m_{n}} \leq C_{35} \frac{M_{n_{4}}}{m_{n_{4}}}
$$

with:

$$
C_{35}=\exp \left(\frac{2\left(2 C_{33}^{2}\right)^{\theta}-1}{\left(2 C_{33}^{2}\right)^{\theta}-1} \frac{\left(C_{19}^{f}\right)^{\left(n_{2}-1\right) \theta}}{1-\left(C_{19}^{f}\right)^{\theta}}+3 C_{28}^{f, k} \frac{\left(C_{19}^{f}\right)^{\left(n_{2}-1\right) / 2}}{1-\left(C_{19}^{f}\right)^{1 / 2}}\right)
$$

Proof. Since for any $n \geq n_{4},\left(R_{n}\right)$ occurs, then by corollary 4.6 , we have:

$$
\begin{gathered}
\frac{M_{n+1}}{M_{n}} \leq \frac{1+M_{n}^{\theta}}{1-C_{28}^{f, k} M_{n}^{1 / 2}} \frac{\alpha_{n+1}}{\alpha_{n}} \\
\frac{m_{n+1}}{m_{n}} \geq \frac{1-M_{n}^{\theta}}{1+C_{28}^{f, k} M_{n}^{1 / 2}} \frac{\alpha_{n+1}}{\alpha_{n}}
\end{gathered}
$$

Therefore,

$$
\frac{M_{n+1} / m_{n+1}}{M_{n} / m_{n}} \leq \frac{1+M_{n}^{\theta}}{1-M_{n}^{\theta}} \frac{1+C_{28}^{f, k} M_{n}^{1 / 2}}{1-C_{28}^{f, k} M_{n}^{1 / 2}}
$$

Therefore, for any $n \geq n_{4}$, 


$$
\frac{M_{n}}{m_{n}} \leq \frac{M_{n_{4}}}{m_{n_{4}}} \prod_{j=n_{4}}^{+\infty} \frac{1+M_{j}^{\theta}}{1-M_{j}^{\theta}} \frac{1+C_{28}^{f, k} M_{j}^{1 / 2}}{1-C_{28}^{f, k} M_{j}^{1 / 2}}
$$

As in the proof of estimation (20), and since $n_{4} \geq n_{2}$, we have:

$$
\begin{gathered}
\prod_{j=n_{4}}^{+\infty}\left(1+M_{j}^{\theta}\right) \leq \exp \left(\sum_{j=n_{2}}^{+\infty} M_{j}^{\theta}\right) \leq \exp \left(\frac{\left(C_{19}^{f}\right)^{\left(n_{2}-1\right) \theta}}{1-\left(C_{19}^{f}\right)^{\theta}}\right) \\
\prod_{j=n_{4}}^{+\infty}\left(1+C_{28}^{f, k} M_{j}^{1 / 2}\right) \leq \exp \left(C_{28}^{f, k} \sum_{j=n_{2}}^{+\infty} M_{j}^{1 / 2}\right) \leq \exp \left(C_{28}^{f, k} \frac{C_{19}^{f\left(n_{2}-1\right) / 2}}{1-\left(C_{19}^{f}\right)^{1 / 2}}\right) \\
\prod_{j=n_{4}}^{+\infty} \frac{1}{1-C_{28}^{f, k} M_{j}^{1 / 2}} \leq \exp \left(C_{28}^{f, k} \sum_{j=n_{2}}^{+\infty} \frac{M_{j}^{1 / 2}}{1-C_{28}^{f, k} M_{j}^{1 / 2}}\right) \leq \exp \left(2 C_{28}^{f, k} \frac{\left(C_{19}^{f}\right)^{\left(n_{2}-1\right) / 2}}{1-\left(C_{19}^{f}\right)^{1 / 2}}\right)
\end{gathered}
$$

and since, for $j \geq n_{2}, M_{j} \leq 1 /\left(2 C_{33}^{2}\right)$, we get:

$$
\prod_{j=n_{4}}^{+\infty} \frac{1}{1-M_{j}^{\theta}} \leq \exp \left(\sum_{j=n_{2}}^{+\infty} \frac{M_{j}^{\theta}}{1-M_{j}^{\theta}}\right) \leq \exp \left(\frac{\frac{\left(C_{19}^{f}\right)^{\left(n_{2}-1\right) \theta}}{1-\left(C_{19}^{f}\right)^{\theta}}}{1-\frac{1}{\left(2 C_{33}^{2}\right)^{\theta}}}\right)
$$

Therefore,

$$
\frac{M_{n}}{m_{n}} \leq \frac{M_{n_{4}}}{m_{n_{4}}} \exp \left(\frac{2\left(2 C_{33}^{2}\right)^{\theta}-1}{\left(2 C_{33}^{2}\right)^{\theta}-1} \frac{\left(C_{19}^{f}\right)^{\left(n_{2}-1\right) \theta}}{1-\left(C_{19}^{f}\right)^{\theta}}+3 C_{28}^{f, k} \frac{\left(C_{19}^{f}\right)^{\left(n_{2}-1\right) / 2}}{1-\left(C_{19}^{f}\right)^{1 / 2}}\right)
$$

Hence proposition 4.19 .

Proof of estimation (1). By combining propositions 4.17 and 4.19, and since by [14, p. 348],

$|D h|_{0} \leq \sup _{n \geq 0} M_{n} / m_{n}$, we get:

$$
|D h|_{0} \leq C_{36}|D f|_{0}^{\frac{2}{\alpha_{n_{4}}}}
$$

with:

$$
C_{36}=3 C_{35}
$$

We estimate $C_{35}$ : since $(2 x-1) /(x-1)=2+1 /(x-1)$, since $\left(C_{19}^{f}\right)^{\left(n_{2}-1\right) \theta} \leq 1 /\left(2\left(C_{33}\right)^{2}\right)^{\theta}$ and since $\theta \leq 1 / 2$, then:

$$
C_{35} \leq \exp \left(\left(2+\frac{1}{\left(2\left(C_{33}\right)^{2}\right)^{\theta}-1}+3 C_{28}^{f, k}\right) \frac{1}{\left(2\left(C_{33}\right)^{2}\right)^{\theta}\left(1-\left(C_{19}^{f}\right)^{\theta}\right)}\right)
$$

Since $C_{19}^{f} \geq 1$, we get: 


$$
|D h|_{0} \leq C_{37}|D f|_{0}^{\frac{2}{\alpha_{n_{4}}}}
$$

with:

$$
C_{37}=3 e \wedge\left(\left(2+\frac{1}{\left(2\left(C_{33}\right)^{2}\right)^{\theta}-1}+3 C_{28}^{f, k}\right) \frac{1}{\left(2\left(C_{33}\right)^{2}\right)^{\theta}\left(1-\left(C_{19}^{f}\right)^{\theta}\right)}\right)
$$

We estimate $C_{37}$ using expressions of $\theta$ (see (19)), of $C_{19}^{f}$ (see lemma 3.4) and of $C_{33}$ (see (21) and proposition 4.5).

We estimate $\alpha_{n_{4}}$ using propositions $4.15,4.14,4.12,4.11$, and the expressions of $n_{2}$ (see (24)) and estimates of $\theta, C_{19}^{f}$ and $C_{33}$. We get:

$$
|D h|_{0} \leq C_{2}\left(k, \beta, C_{d},|D f|_{0}, W(f),|S f|_{k-3}\right)
$$

where $C_{2}$ is the combination of the following functions:

1. $C_{19}^{f}=\left(1+e \wedge\left(-6 \sqrt{2} e^{2 W(f)}\left(|S f|_{0}^{1 / 2} \vee 1\right)\right)\right)^{-1 / 2}$

(since $|S f|_{0} \leq|S f|_{k-3}$, we can estimate $C_{19}^{f}$ in function of $W(f),|S f|_{k-3}$ ).

2. $C_{28}^{f, k}=(k+3)^{(k+3) !} e^{(k+3) ! W(f)}\left(\max \left(1,|S f|_{k-3}\right)\right)^{(k+1) !}$

3. $\theta=\min \left(1 / 2,\left(\frac{3+\beta}{4}\right)\left(-1+\sqrt{1+\frac{2(k-(2 \beta+1))}{(3+\beta)^{2}}}\right)\right)$

4. $C_{33}=\max \left(e^{\frac{2}{1-\left(C_{19^{f}}^{f}\right.}},\left(4 C_{28}^{f, k}\right) \frac{1}{(1+\beta+\theta)(1+\theta)-1}\right)$

5. $n_{2}=\left\lfloor\max \left(-\frac{\log \left(2 C_{33}^{2}\right)}{\log C_{19}^{f}}+\frac{2 \log \left(1 / C_{d}\right)}{\theta \log 2}+2,2+\frac{\left(2 C_{2}^{f, k}\right)}{\log \left(\left(C_{19}^{f}\right)^{1 / 2}\right)}\right)\right\rfloor$

6. $\beta_{1}=\beta+\frac{2 \log \left(1 / C_{d}\right)}{\left(n_{2}-1\right) \log 2}$

7. $n_{3}=\left\lceil\frac{1}{\log \left(\frac{(k+1) / 2-\theta}{1+\beta_{1}}\right)}\left(n_{2}(1+\log (1+\beta))+\log \left(\frac{\left(n_{2}+1\right) \log \left(1 / C_{d}\right)}{\log 2}\right)\right)\right\rceil$

8. $\rho=\frac{\beta_{1}}{\frac{k-1}{2}-\theta}$

9. $\alpha_{n_{4}}^{\prime}=C_{d} \wedge\left(e \wedge\left(\left(n_{3}+1+\frac{\rho}{1-\rho}\right)\left(1+\beta_{1}\right)\right)\right)$

10. $C_{37}=3 e \wedge\left(\left(2+\frac{1}{\left(2\left(C_{33}\right)^{2}\right)^{\theta}-1}+3 C_{28}^{f, k}\right) \frac{1}{\left(2\left(C_{33}\right)^{2}\right)^{\theta}\left(1-\left(C_{19}^{f}\right)^{\theta}\right)}\right)$

11. $|D h|_{0} \leq C_{37}|D f|_{0}^{\frac{2}{\alpha_{n_{4}}}}$

Note that we have a bound $\alpha_{n_{4}}^{\prime} \leq \alpha_{n_{4}}$, but we do not know the value of $\alpha_{n_{4}}$. 


\subsection{Proof of estimation (2).}

In order to obtain relatively simple estimates, we can take the parameter $\theta$ (defined in (19)) either vanishingly close to 0 (estimation (3)), or fixed independently of the other parameters (estimation (2)).

In the latter case, we need to assume that $k-2 \beta-1$ is sufficiently large, in order to keep $(k+1) / 2-\theta \geq(1+\beta+\theta)(1+\theta)$. To illustrate this case, we take $\theta=1 / 2$, which requires $k \geq 3 \beta+9 / 2$ (for any fixed $\theta$, we cannot obtain an assumption of the form $k \geq 2 \beta+u$ for some number $u$ : we necessarily have $k \geq \lambda \beta+u$ with $\lambda>2$ ).

To simplify the function $C_{2}$, we successively estimate $C_{35}, \alpha_{n_{4}}^{\prime}$ and $n_{2}$.

Lemma 4.20. Let $C_{35}$ and $C_{33}$ defined in proposition 4.19 and (21) respectively. We have:

$$
C_{35} \leq \exp \left(C_{33}^{\frac{3 \beta+1}{2}}\right)
$$

Proof. For any $x \geq 2$,

$$
(2 x-1) /(x-1)=2+1 /(x-1) \leq 3
$$

Since $C_{33} \geq C_{32} \geq e \geq 2$, then

$$
\begin{gathered}
\exp \left(\frac{2\left(2 C_{33}\right)^{1 / 2}-1}{\left(2 C_{33}\right)^{1 / 2}-1}\left(1 / C_{19}^{f}\right)^{1 / 2} \frac{\left(C_{19}^{f}\right)^{n_{2} / 2}}{1-\left(C_{19}^{f}\right)^{1 / 2}}+3 C_{28}^{f, k}\left(1 / C_{19}^{f}\right)^{1 / 2} \frac{\left(C_{19}^{f}\right)^{n_{2} / 2}}{1-\left(C_{19}^{f}\right)^{1 / 2}}\right) \\
\leq \exp \left(3\left(1+C_{28}^{f, k}\right)\left(1 / C_{19}^{f}\right)^{1 / 2} \frac{\left(C_{19}^{f}\right)^{n_{2} / 2}}{1-\left(C_{19}^{f}\right)^{1 / 2}}\right)
\end{gathered}
$$

On the other hand, since $n_{2} \geq \frac{-\log \left(2\left(C_{33}\right)^{2} / C_{19}^{f}\right)}{\log C_{19}^{f}}$ and $C_{19}^{f}<1$, then:

$$
e^{\frac{n_{2}}{2} \log C_{19}^{f}} \leq\left(2\left(C_{33}\right)^{2} / C_{19}^{f}\right)^{-1 / 2}
$$

Therefore,

$$
\begin{gathered}
\left(1 / C_{19}^{f}\right)^{1 / 2} \frac{\left(C_{19}^{f}\right)^{n_{2} / 2}}{1-\left(C_{19}^{f}\right)^{1 / 2}}=\left(1 / C_{19}^{f}\right)^{1 / 2} \frac{e^{\frac{n_{2}}{2} \log C_{19}^{f}}}{1-\left(C_{19}^{f}\right)^{1 / 2}} \\
\leq\left(1 / C_{19}^{f}\right)^{1 / 2} \frac{\left(2\left(C_{33}\right)^{2} / C_{19}^{f}\right)^{-1 / 2}}{1-\left(C_{19}^{f}\right)^{1 / 2}}=\frac{1}{\sqrt{2} C_{33}\left(1-\left(C_{19}^{f}\right)^{1 / 2}\right)}
\end{gathered}
$$

Moreover, since $C_{28}^{f, k} \geq 1 /(\sqrt{2}-1)$ then $\left(1+C_{28}^{f, k}\right) / \sqrt{2} \leq C_{28}^{f, k}$. Therefore,

$$
\exp \left(3\left(1+C_{28}^{f, k}\right)\left(1 / C_{19}^{f}\right)^{1 / 2} \frac{\left(C_{19}^{f}\right)^{n_{2} / 2}}{1-\left(C_{19}^{f}\right)^{1 / 2}}\right) \leq \exp \left(\frac{3 C_{28}^{f, k}}{C_{33}\left(1-\left(C_{19}^{f}\right)^{1 / 2}\right)}\right)
$$

Since $C_{33}^{\frac{3}{2}(3 / 2+\beta)-1} \geq 4 C_{28}^{f, k}$, then

$$
\frac{C_{28}^{f, k}}{C_{33}} \leq \frac{1}{4} C_{33}^{\frac{1}{4}+\frac{3}{2} \beta}
$$


We also have:

$$
C_{33}^{\frac{1}{4}} \geq e^{\frac{1 / 2}{1-\left(C_{19}^{f}\right)^{1 / 2}}} \geq \frac{1}{4} \frac{1}{1-\left(C_{19}^{f}\right)^{1 / 2}}
$$

We obtain:

$$
\exp \left(\frac{3 C_{28}^{f, k}}{C_{33}\left(1-\left(C_{19}^{f}\right)^{1 / 2}\right)}\right) \leq\left(e^{\frac{1}{4} C_{33}^{\frac{1}{4}+\frac{3}{2} \beta} \frac{1}{1-\left(C_{19}^{f}\right)^{1 / 2}}}\right) \leq \exp \left(C_{33}^{\frac{3 \beta+1}{2}}\right)
$$

Lemma 4.21. Let $\alpha_{n_{4}}^{\prime}$ defined page 23 and $n_{2}$ defined in (24). We have:

$$
\frac{1}{\alpha_{n_{4}}^{\prime}} \leq\left(\frac{1}{C_{d}}\right) \wedge e \wedge\left((\beta+3 / 2)\left(2+\frac{n_{2}}{\log (3 / 2)}\left(2+\log (1+\beta)+\log \log \left(1 / C_{d}\right)\right)\right)\right)
$$

Proof. Since $\beta_{1} \leq \beta+1 / 2$, and $\theta=1 / 2$, then

$$
\log \left(\frac{(k+1) / 2-\theta}{1+\beta_{1}}\right) \geq \log (3 / 2)
$$

Therefore,

$$
n_{3} \leq \frac{n_{2}}{\log (3 / 2)}\left(1+\log (1+\beta)+\frac{\log \left(\left(n_{2}+1\right) \log \left(1 / C_{d}\right)\right)}{n_{2} \log 2}\right)
$$

We have $\log \left(n_{2}+1\right) / n_{2} \leq 1$ and $n_{2} \log 2 \geq 1$.

Moreover, $\rho \leq 2 / 3$, and so $\rho /(1-\rho) \leq 2$. Hence the lemma.

Lemma 4.22. Let $n_{2}$ defined in (24). We have:

$$
n_{2} \leq C_{38}\left(W(f),|S f|_{0}\right)(k+4) !\left(1+\log \left(\max \left(1,|S f|_{k-3}\right)\right)\right)\left(1+\log \left(1 / C_{d}\right)\right)
$$

with:

$$
C_{38}\left(W(f),|S f|_{0}\right)=e^{(2)} \wedge\left(3 W(f)+2 \log \left(\max \left(1,|S f|_{0}\right)\right)+4\right)
$$

Proof. In order to estimate $n_{2}$, we need to estimate $C_{33}$ (see page 23). We distinguish the cases $C_{33}=\left(4 C_{28}^{f, k}\right)^{\frac{1}{(1+\beta+\theta)(1+\theta)-1}}$ and $C_{33}=C_{32}^{f, k}$.

First, we suppose $C_{33}=\left(4 C_{28}^{f, k}\right)^{\frac{1}{(1+\beta+\theta)(1+\theta)-1}}$. We have: $C_{33} \leq 4 C_{28}^{f, k}$. Therefore,

$$
n_{2} \leq 2+\frac{\log \left(2\left(4 C_{28}^{f, k}\right)^{2}\right)}{-\log \left(C_{19}^{f}\right)}+\frac{4}{\log 2} \log \left(1 / C_{d}\right)
$$

Moreover (see page 23),

$$
\log \left(C_{28}^{f, k}\right) \leq(k+3) !\left[\log (k+3)+W(f)+\max \left(1,|S f|_{k-3}\right)\right]
$$

And for any $x>-1, \log (1+x) \geq x /(1+x)$. Therefore,

$$
\frac{1}{-\log \left(C_{19}^{f}\right)} \leq \frac{1}{\frac{1}{2} \log \left(1+e^{-C_{22}^{f}}\right)} \leq 2+2 e^{C_{22}^{f}}
$$


Therefore,

$$
\begin{gathered}
n_{2} \leq 2+2\left(1+e^{C_{22}^{f}}\right)\left[5(\log 2)+2(k+3) !\left(W(f)+\log (k+3)+\log \left(\max \left(1,|S f|_{k-3}\right)\right)\right)\right] \\
+\frac{4}{\log 2} \log \left(1 / C_{d}\right)
\end{gathered}
$$

Moreover, by relation (11),

$$
4 e^{C_{22}^{f}} \leq \exp \left(\exp \left(2 W(f)+\log \left(6 \sqrt{2}\left(\max \left(1,|S f|_{0}\right)\right)^{1 / 2}\right)+1\right)\right)=C_{39}^{f}
$$

Moreover, $(k+4) ! / 3 \geq 2+5 \log 2$ and $2(k+4) / 3 \geq 2 \log (k+3)$. Therefore,

$$
(k+4) ! \geq 2+5 \log 2+2(k+3) ! \log (k+3)
$$

Moreover, $2\left(1+e^{C_{22}^{f}}\right) \leq 4 e^{C_{22}^{f}}$. We get:

$$
n_{2} \leq(k+4) ! C_{39}^{f}\left(W(f)+1+\log \left(\max \left(1,|S f|_{k-3}\right)\right)+\frac{4}{\log 2} \log \left(1 / C_{d}\right)\right.
$$

By using that $\log (6 \sqrt{2})+1 \leq 4$ and $4 / \log 2 \leq(k+4)$ !, we obtain the estimation of lemma 4.22.

If $C_{33}=C_{32}$, we need the following lemma:

Lemma 4.23. Let $C_{33}$ given by (21) and $C_{34}$ given by (22). If $C_{33}=C_{32}$, then

$$
C_{34} \leq 40 e^{2 C_{22}^{f}}
$$

Proof. We have:

$$
C_{34}=\frac{-\log \left(2\left(C_{33}\right)^{2} / C_{19}^{f}\right)}{\log C_{19}^{f}}=1+\frac{\log 2}{\frac{1}{2} \log \left(1+e^{-C_{22}^{f}}\right)}+\frac{4}{\left(1-\left(1+e^{-C_{22}^{f}}\right)^{-1 / 4}\right) \frac{1}{2} \log \left(1+e^{-C_{22}^{f}}\right)}
$$

Since for any $x>-1, \log (1+x) \geq x /(1+x)$, then:

$$
\frac{1}{\log \left(1+e^{-C_{22}^{f}}\right)} \leq e^{C_{22}^{f}}\left(1+e^{-C_{22}^{f}}\right)
$$

On the other hand,

$$
1-\left(1+e^{-C_{22}^{f}}\right)^{-1 / 4}=\int_{0}^{e^{-C_{22}^{f}}} \frac{1}{4} \frac{1}{(1+t)^{5 / 4}} d t \geq \frac{e^{-C_{22}^{f}}}{4\left(1+e^{-C_{22}^{f}}\right)^{5 / 4}}
$$

Therefore,

$$
C_{34} \leq 8\left(1+e^{-C_{22}^{f}}\right)^{9 / 4} e^{2 C_{22}^{f}}\left(\frac{1}{8\left(1+e^{-C_{22}^{f}}\right) e^{2 C_{22}^{f}}}+\frac{\log 2}{4 e^{C_{22}^{f}}}+4\right)
$$

Since $C_{22}^{f} \geq 6 \sqrt{2}$ (see expression (11)), then:

$$
e^{-C_{22}^{f}} \leq 2.07 \times 10^{-4}
$$


Therefore,

$$
\frac{1}{8\left(1+e^{-C_{22}^{f}}\right) e^{2 C_{22}^{f}}}+\frac{\log 2}{4 e^{C_{22}^{f}}}+4 \leq 4.1
$$

Therefore,

$$
C_{34} \leq 40 e^{2 C_{22}^{f}}
$$

Assuming that $C_{33}=C_{32}$, if $n_{2}=\left\lfloor 2+\frac{\left(2 C_{28}^{f, k}\right)}{\log \left(\left(C_{19}^{f}\right)^{1 / 2}\right)}\right\rfloor$ then we can follow the first part of the proof and we still obtain the estimate of lemma 4.22 .

If $n_{2}=\left\lfloor-\frac{\log \left(2 C_{33}^{2}\right)}{\log C_{19}^{f}}+\frac{2 \log \left(1 / C_{d}\right)}{\theta \log 2}+2\right\rfloor$, then:

$$
\frac{-\log \left(2\left(C_{33}\right)^{2} / C_{19}^{f}\right)}{\log C_{19}^{f}}=C_{34} \leq 20 e^{2 C_{22}^{f}} \leq 2\left(C_{39}^{f}\right)^{2} \leq C_{38}^{f}
$$

Therefore,

$$
n_{2} \leq-\frac{\log \left(2 C_{33}^{2}\right)}{\log C_{19}^{f}}+\frac{2 \log \left(1 / C_{d}\right)}{\theta \log 2}+2 \leq C_{38}^{f}+\frac{4 \log \left(1 / C_{d}\right)}{\log 2}+1
$$

The estimation of lemma 4.22 still holds. This completes the proof of lemma 4.22.

Now, we show estimation (2). We denote $\log ^{(2)}(x)=\log \log x$. By applying estimation (36) and lemma 4.21, we have:

$|D h|_{0} \leq e^{(3)} \wedge\left(\log ^{(2)}\left(C_{36}\right)+\log ^{(2)}\left(|D f|_{0}\right)+\log 2+\log ^{(2)}\left(1 / C_{d}\right)+(\beta+3 / 2)\left(2+\frac{n_{2}}{\log (3 / 2)}\left(2+\beta+\log ^{(2)}\left(1 / C_{d}\right)\right)\right)\right)$

By lemma 4.20, we have:

$$
\log ^{(2)}\left(C_{36}\right) \leq \log ^{(2)}\left(3 C_{35}\right) \leq \log \left(\log 3+C_{33}^{\frac{3 \beta+1}{2}}\right)
$$

Moreover, since $C_{33} \geq e, \frac{3 \beta+1}{2} \geq 1 / 2$ and $e\left(e^{1 / 2}-1\right) \geq \log 3$, then

$$
\log 3+C_{33}^{\frac{3 \beta+1}{2}} \leq C_{33}^{\frac{3 \beta+1}{2}+1}
$$

Therefore,

$$
\log ^{(2)}\left(C_{36}\right) \leq\left(\frac{3 \beta+1}{2}+1\right) C_{33}
$$

Moreover, by estimation (38), $e^{-C_{22}^{f}} \leq 2.07 \times 10^{-4}$, and therefore, $-\log \left(C_{19}^{f}\right) \leq 2 / 3$ (see expression (10)). By applying the definition of $n_{2}$ (page 15), we get:

$$
\log ^{(2)}\left(C_{36}\right) \leq 3 \frac{(\beta+1)}{2} \log C_{33} \leq \frac{\beta+1}{2} n_{2}
$$

Moreover, 


$$
\frac{\beta+1}{2} n_{2}+\left(\frac{\beta+3 / 2}{\log (3 / 2)}\right)(\beta+2) n_{2}+2(\beta+3 / 2) \leq \frac{\beta+3 / 2}{\log (3 / 2)}(\beta+3)\left(n_{2}+2\right)
$$

Therefore,

$|D h|_{0} \leq e^{(3)} \wedge\left(\frac{2}{\log (3 / 2)}(\beta+3 / 2)(\beta+3)\left(n_{2}+2\right)\left(1+\log ^{(2)}\left(|D f|_{0}\right)\right)\left(1+\log ^{(2)}\left(1 / C_{d}\right)\right)\right)$

We have: $n_{2}+2 \leq 2 n_{2}$. By lemma 4.22 , and since $4 / \log (3 / 2) \leq 10$, we get:

$|D h|_{0} \leq e^{(3)} \wedge\left((\beta+3 / 2)(\beta+3)(k+4) ! C_{5}\left(1+\log \left(1 / C_{d}\right)\right)^{2}\left(1+\log \left(\max \left(1,|S f|_{k-3}\right)\right)\right)\right)$

with $\left.C_{5}=10\left(1+\log ^{(2)}\left(|D f|_{0}\right)\right) e^{(2)} \wedge\left(3 W(f)+2 \log \left(\max \left(1,|S f|_{0}\right)\right)\right)+2\right)$.

This estimation of $|D h|_{0}$ is increasing with $k$. Therefore, to obtain a bound as low as we can, we take $k=\lceil 3 \beta+9 / 2\rceil$. We obtain:

$$
|D h|_{0} \leq e^{(3)} \wedge\left(C_{3}[\beta] C_{4}\left[C_{d}\right] C_{5}\left[|D f|_{0}, W(f),|S f|_{0}\right] C_{6}\left[|S f|_{[3 \beta+3 / 2]}\right]\right)
$$

with:

1. $C_{3}[\beta]=(\lceil 3 \beta+21 / 2\rceil)$ !

2. $C_{4}\left[C_{d}\right]=\left(1+\log \left(1 / C_{d}\right)\right)^{2}$

3. $\left.C_{5}\left[|D f|_{0}, W(f),|S f|_{0}\right]=10\left(1+\log ^{(2)}\left(|D f|_{0}\right)\right) e^{(2)} \wedge\left(3 W(f)+2 \log \left(\max \left(1,|S f|_{0}\right)\right)\right)+4\right)$

4. $C_{6}\left[|S f|_{[3 \beta+3 / 2\rceil}\right]=1+\log \left(\max \left(1,|S f|_{[3 \beta+3 / 2\rceil}\right)\right)$

\subsection{Proof of estimation (3)}

Let $\delta=k-2 \beta-1$ and $\beta>0$. We make a Taylor expansion with $\delta \rightarrow 0$ (since $k \geq 3$, this implies automatically $\beta>0$ ). To estimate $|D h|_{0}$, we successively estimate $n_{2}, n_{3}$, $\rho /(1-\rho)$ and $\alpha_{n_{4}}^{\prime}$.

We have:

$$
\theta=\frac{\delta}{4(3+\beta)}+o(\delta)
$$

Since $\beta>0$, then for $\delta$ sufficiently small, $C_{33}=e^{\frac{2}{1-\left(C_{19}^{f}\right)^{\theta}}}$. This makes the dependence on $k$ and $|S f|_{k-3}$ disappear. Moreover,

$$
\begin{gathered}
C_{33}=e \wedge\left(\frac{2}{\theta \log \left(1 / C_{19}^{f}\right)+o(\theta)}\right) \\
n_{2}=\left(\frac{4}{\left(\log C_{19}^{f}\right)^{2}}+\frac{2 \log \left(1 / C_{d}\right)}{\log 2}\right) \frac{1}{\theta}+o\left(\frac{1}{\theta}\right)
\end{gathered}
$$

We denote $C_{40}=\frac{4}{\left(\log C_{19}^{f}\right)^{2}}$ and $C_{41}=\frac{2 \log \left(1 / C_{d}\right)}{\log 2}$. 
Since

$$
\beta_{1}=\beta+\frac{C_{41}}{n_{2}}+o(\theta)
$$

then

$$
\log \left(\frac{\frac{k+1}{2}-\theta}{1+\beta_{1}}\right)=\frac{\frac{k+1}{2}-\theta}{1+\beta_{1}}-1+o(\theta)=\frac{\delta / 2-\theta-C_{41} / n_{2}}{1+\beta_{1}}+o(\theta)
$$

Therefore,

$$
\begin{gathered}
n_{3}=n_{2}(1+\log (1+\beta))\left(\frac{1+\beta_{1}}{\delta / 2-\theta-C_{41} / n_{2}}\right)+o\left(\frac{1}{\theta^{2}}\right) \\
n_{3} \leq(1+\beta)^{2} \frac{n_{2}^{2}}{\delta n_{2} / 2-\theta n_{2}-C_{41}}+o\left(\frac{1}{\theta^{2}}\right)
\end{gathered}
$$

Moreover, $\theta n_{2}=C_{41}+C_{40}+o(1)$ and $\delta n_{2}=4(3+\beta)\left(C_{41}+C_{40}\right)+o(1)$. Therefore,

$$
n_{3} \leq \frac{(1+\beta)^{2}\left(C_{41}+C_{40}\right)}{\theta^{2}(4+2 \beta)}+o\left(\frac{1}{\theta^{2}}\right)
$$

Moreover,

$$
\rho=\frac{\beta_{1}}{\frac{k-1}{2}-\theta}=\frac{\beta+\frac{C_{41}}{C_{41}+C_{40}} \theta+o(\theta)}{\beta+\delta / 2-\theta}=1-\frac{\theta}{\beta}\left(2(3+\beta)-1-\frac{C_{41}}{C_{41}+C_{40}}\right)+o(\theta)
$$

Therefore, $\rho /(1-\rho)=o\left(1 / \delta^{2}\right)\left(\right.$ we recall that $\left.1 / \delta=o\left(1 / \delta^{2}\right)\right)$.

Let

$$
C_{7}\left[k, C_{d},|D f|_{0}, W(f),|S f|_{0}\right]=\frac{(k+5)^{2}(k+1)^{3}}{2 k \log 2}\left(\frac{2 \log 2}{\left(\log C_{19}^{f}\right)^{2}}+\log \left(1 / C_{d}\right)\right)
$$

Since $k=2 \beta+1+\delta$, and by applying estimation (40), we have:

$$
\alpha_{n_{4}}^{\prime} \geq C_{d} \wedge\left(e \wedge\left(\frac{C_{7}}{\delta^{2}}+o\left(\frac{1}{\delta^{2}}\right)\right)\right)
$$

Therefore,

$$
\begin{gathered}
|D h|_{0} \leq C_{37}|D f|_{0} \wedge\left(\left(1 / C_{d}\right) \wedge\left(e \wedge\left(\frac{1}{\delta^{2}} C_{7}+o\left(1 / \delta^{2}\right)\right)\right)\right) \\
|D h|_{0} \leq C_{37}|D f|_{0} \wedge\left(e \wedge\left(e \wedge\left(\log \log \left(1 / C_{d}\right)+\frac{1}{\delta^{2}} C_{7}+o\left(1 / \delta^{2}\right)\right)\right)\right)
\end{gathered}
$$

Since $\log ^{(2)}\left(1 / C_{d}\right)=o\left(1 / \delta^{2}\right)$, then

$$
|D h|_{0} \leq C_{37}|D f|_{0} \wedge\left(e \wedge\left(e \wedge\left(\frac{1}{\delta^{2}} C_{7}+o\left(1 / \delta^{2}\right)\right)\right)\right)
$$

Likewise, since $\left.|\log \log | D f\right|_{0} \mid \leq e^{o\left(1 / \delta^{2}\right)}$ and $\left|\log C_{37}\right| \leq e \wedge e \wedge\left(o\left(1 / \delta^{2}\right)\right)$. We conclude: 


$$
|D h|_{0} \leq e^{(3)} \wedge\left(\frac{1}{\delta^{2}} C_{7}\left[k, C_{d}, W(f),|S f|_{0}\right]+o\left(1 / \delta^{2}\right)\right)
$$

with:

$$
C_{7}\left[k, C_{d}, W(f),|S f|_{0}\right]=\frac{(k+5)^{2}(k+1)^{3}}{2 k \log 2}\left(\frac{2 \log 2}{\left(\log C_{19}^{f}\right)^{2}}+\log \left(1 / C_{d}\right)\right)
$$

We recall that:

$$
C_{19}^{f}=\left(1+e \wedge\left(-6 \sqrt{2} e^{2 W(f)}\left(|S f|_{0}^{1 / 2} \vee 1\right)\right)\right)^{-1 / 2}
$$

In estimations (2) and (3), three iterations of the exponential appear. This calls for explanation. A first exponential comes from the estimation $\left|D f^{n}\right|_{0} \leq C|D f|_{0}^{2 / \alpha_{n_{4}}}$, where $n_{4}$ is the rank above which the "favorable" case always occurs. A second exponential comes from writing $\alpha_{n_{4}}=\alpha_{0}^{\prod_{n=0}^{n_{4}-1}\left(\frac{1}{1-\eta_{n}}\right)}$. We bound each $\frac{1}{1-\eta_{n}}$ using the Diophantine condition, and a third exponential comes from the estimation $\prod_{n \in E_{2}}\left(\frac{1}{1-\eta_{n}}\right) \leq C^{n_{3}-n_{2}}$, where $E_{2}$ is the set and $n_{3}-n_{2}$ is the number of "unfavorable" cases.

This number is bounded $\log$ arithmically, by $C \log C_{33}$. However, $C_{33}$ is bounded by an exponential of the parameters. Indeed, when $\delta$ is small, $C_{33} \sim e^{\frac{1}{\delta}}$, which gives estimation (3). Otherwise, $C_{33} \sim C_{28}^{f, k}$. In this case, $C_{28}^{f, k} \sim C^{k}$. Indeed, in lemma 14, we need $k-1$ iterations to estimate $\left|D^{k-1} \log D f^{p}(x)\right|_{0}\left(p \leq q_{n+1}\right)$, an estimation that, in turn, gives an estimate of $C_{28}^{f, k}$. This gives estimation (2). Thus, we have explained the occurence of three exponentials in the estimates.

Since the number of "unfavorable" cases drives the dominant term of these estimates, they can be substantially improved when the "favorable" case always occurs. In remark 1.5, we make this assumption, together with the assumption $k \geq 3 \beta+9 / 2$. Thus, we can take $\theta=1 / 2$, and a sufficient condition for the occurrence of the "favorable" case is:

$$
\frac{\alpha_{n+1}}{\alpha_{n}} \geq C_{28}^{f, k}\left(C_{19}^{f}\right)^{(n-1) \frac{k}{2}}=C_{8}\left(n, k, \beta, W(f),|S f|_{k-3}\right)
$$

which decreases geometrically with $n$.

We recall that:

$$
\begin{gathered}
C_{19}^{f}=\left(1+e \wedge\left(-6 \sqrt{2} e^{2 W(f)}\left(|S f|_{0}^{1 / 2} \vee 1\right)\right)\right)^{-1 / 2} \\
C_{28}^{f, k}=(k+3)^{(k+3) !} e^{(k+3) ! W(f)}\left(\max \left(1,|S f|_{k-3}\right)\right)^{(k+1) !}
\end{gathered}
$$

We obtain the following estimation:

$$
|D h|_{0} \leq \exp \left(C_{9}\left[k, W(f),|S f|_{k-3}\right]^{C_{10}(\beta)}\right)|D f|_{0}^{2}
$$

with:

$$
C_{9}\left[k, W(f),|S f|_{k-3}\right]=\max \left(e^{\frac{2}{1-\left(C_{19}^{f}\right)^{1 / 2}}}, 4 C_{28}^{f, k}\right)
$$




$$
C_{10}[\beta]=\frac{3 \beta+1}{2}
$$

Finally, note that numbers of constant type do not always satisfy (4) for any $n$ (they only satisfy it above some rank). Moreover, there are numbers satisfying (4) that are not of constant type.

\section{$5 \quad C^{k}$ estimations}

In this section, we compute estimates of higher order derivatives of the conjugacy $h$ in function of bounds on the first derivative of $h$. We compute the values of some of the constants appearing in Yoccoz's proof [14] (we do not compute the dependency in $k$ ). However, in order to obtain our result, we need to slightly modify the proof of one proposition (proposition 5.10). If we strictly followed Yoccoz's proof, we would find an estimate that depends on the $C^{1}$-norm of $h$, and on $k, \beta, C_{d}, W(f),|S f|_{k-3},\left|D^{k-1} \log D f\right|_{0}$, but this estimate would diverge as $f$ gets closer to a rotation. Moreover, we need to elaborate on the end of his proof.

The proof has four steps. We let real numbers $0 \leq \gamma_{0}<\gamma_{1}<g\left(\gamma_{0}\right)$, with

$g\left(\gamma_{0}\right)=\left((1+\beta) \gamma_{0}+k-(2+\beta)\right) /(2+\beta)$, and we let an integer $N$. In the first three steps, we compute $\left\|\log D f^{N}\right\|_{\gamma_{1}}$ in function of $\sup _{p \geq 0}\left\|\log D f^{p}\right\|_{\gamma_{0}}$ (estimation (41)). In the first step, using convexity estimations (proposition 5.7) and a consequence of the Faa-di-Bruno formula (lemma 5.6), we establish an estimation of $\left\|\log D f^{q_{s}}\right\|_{\gamma}$ for $0 \leq \gamma \leq k-1$ (lemma 5.8).

In the second step, we obtain an estimation of $\left\|\log D f^{n q_{s}}\right\|_{\gamma}, 0 \leq n \leq q_{s+1} / q_{s}$ for $0 \leq \gamma \leq \gamma_{1}$ (estimation (54)).

In the third step, we write $N=\sum_{s=0}^{S} b_{s} q_{s}$, with $b_{s}$ integers satisfying $0 \leq b_{s} \leq$ $q_{s+1} / q_{s}$, in order to get an estimation of $\left\|\log D f^{N}\right\|_{\gamma_{1}}$ in function of $\sup _{p \geq 0}\left\|\log D f^{p}\right\|_{\gamma_{0}}$. Thus, in these three steps, the aim is to establish the following proposition:

Proposition 5.1. Let $0 \leq \gamma_{0}<\gamma_{1}<g\left(\gamma_{0}\right)=\frac{(1+\beta) \gamma_{0}+k-(2+\beta)}{2+\beta}$. We have:

$$
\left\|\log D f^{N}\right\|_{\gamma_{1}} \leq e \wedge\left(C_{72}(k, \beta)\left(C_{73}^{f, k}+4 k^{2} \log \left(C_{d}^{-1}\right)+24 k(k+1) \sup _{p \geq 0}\left\|\log D f^{p}\right\|_{\gamma_{0}}\right)^{4}\right)
$$

The expressions of $C_{72}$ and $C_{73}^{f, k}$ are given page 45 .

In the fourth step, we iterate this reasoning: the inductive step is given by proposition 5.1: if we have an estimate of $\sup _{N \geq 0}\left\|\log D f^{N}\right\|_{\gamma_{i}}$, then we can get an estimate of $\sup _{N \geq 0}\left\|\log D f^{N}\right\|_{\gamma_{i+1}}$ for $\gamma_{i}<\gamma_{i+1}<g\left(\gamma_{i}\right)$. We can initiate the induction with $\gamma_{0}=0$, because we have $C^{1}$ estimates. We take $\gamma_{i+1}=\frac{1}{2}\left(g\left(\gamma_{i}\right)+\gamma_{i}\right)$ and we have:

$\lim _{i \rightarrow+\infty} \gamma_{i}=k-2-\beta$. Thus, we can obtain an estimation of $\|D h\|_{k-2-\beta-\eta}$.

In all the rest of the paper, we denote:

$$
M^{\prime}=\exp \left(\sup _{i \geq 0}\left|\log D\left(f^{i}\right)\right|_{0}\right)
$$




$$
M=\exp \left(\sup _{i \geq 0}\left\|\log D\left(f^{i}\right)\right\|_{\gamma_{0}}\right)
$$

Note that $M \geq M^{\prime} \geq 1$.

\subsection{Estimation of $\left\|\log D f^{q_{s}}\right\|_{\gamma}, 0 \leq \gamma \leq k-1$.}

The following lemma is a converse of the implication used in [14, p. 348], according to which if $M_{n} / m_{n}$ is bounded, then the conjugacy of $f$ to a rotation is $C^{1}$ :

Lemma 5.2. Let $M_{n}=\sup _{x \in \mathbb{T}^{1}}\left|f^{q_{n}}(x)-x\right|, m_{n}=\inf _{x \in \mathbb{T}^{1}}\left|f^{q_{n}}(x)-x\right|$ and $M^{\prime}=\exp \left(\sup _{i \geq 0}\left|\log D\left(f^{i}\right)\right|_{0}\right)$. Then we have the following estimation:

$$
\frac{M_{n}}{m_{n}} \leq M^{\prime}
$$

Proof. Let $\epsilon>0, x, y$ such that $M_{n}=\left|f^{q_{n}}(x)-x\right|$ and $m_{n}=\left|f^{q_{n}}(y)-y\right|$. Since $f^{p}(y)_{p \geq 0}$ is dense in $\mathbb{T}^{1}$, then there is a positive integer $l$ such that $\left|f^{l}(y)-x\right| \leq \min \left(\frac{\epsilon}{\mid D f^{\left.q_{n}\right|_{0}}}, \epsilon\right)$.

Then we obtain:

$\left|f^{q_{n}}(x)-x\right| \leq\left|f^{q_{n}}(x)-f^{q_{n}}\left(f^{l}(y)\right)\right|+\left|f^{l}\left(f^{q_{n}}(y)\right)-f^{l}(y)\right|+\left|f^{l}(y)-x\right| \leq\left|D f^{l}\right|_{0}\left|f^{q_{n}}(y)-y\right|+2 \epsilon \leq M^{\prime} m_{n}+2 \epsilon$ for all $\epsilon>0$. Hence the lemma.

The $C^{\gamma}$-norms, when $\gamma$ varies in $\mathbb{R}^{+}$, are related with each other by convexity inequalities (also called interpolation inequalities):

Proposition 5.3. Let $\gamma_{2}, \gamma_{3} \in \mathbb{R}^{+}$with $0 \leq \gamma_{2} \leq \gamma_{3}$ and $\gamma_{3}>0$. For any $\phi \in C^{\gamma_{3}}\left(\mathbb{T}^{1}\right)$, we have:

$$
\|\phi\|_{\gamma_{2}} \leq C_{42}\left(\gamma_{3}\right)\|\phi\|_{0}^{\frac{\gamma_{3}-\gamma_{2}}{\gamma_{3}}}\|\phi\|_{\gamma_{3}}^{\frac{\gamma_{2}}{\gamma_{3}}}
$$

with $C_{42}\left(\gamma_{3}\right)=2^{\left(\left\lfloor\gamma_{3}\right\rfloor+1\right)^{2}+1}$.

Proof. See appendix.

Using these convexity inequalities, we establish various relations, among which is the important corollary 5.5, which relate the norms of $\log D f^{n}$ and those of $D f^{n}-1$.

For $0 \leq \gamma_{1} \leq \gamma_{2} \leq k-1, \gamma_{2} \neq 0$, and $n \in \mathbb{Z}$, proposition 5.3 gives:

$$
\begin{aligned}
& \left\|\log D f^{n}\right\|_{\gamma_{1}} \leq C_{43}\left\|\log D f^{n}\right\|_{\gamma_{2}}^{\gamma_{1} / \gamma_{2}} \\
& \left\|D f^{n}-1\right\|_{\gamma_{1}} \leq C_{44}\left\|D f^{n}-1\right\|_{\gamma_{2}}^{\gamma_{1} / \gamma_{2}}
\end{aligned}
$$

with $C_{43}\left(\gamma_{1}, \gamma_{2}\right)=C_{42}\left(\gamma_{2}\right)\left(\log M^{\prime}\right)^{1-\gamma_{1} / \gamma_{2}}$ and $C_{44}\left(\gamma_{1}, \gamma_{2}\right)=C_{42}\left(\gamma_{2}\right)\left(M^{\prime}+1\right)^{1-\gamma_{1} / \gamma_{2}}$.

For $n \geq 0, j \in \mathbb{Z}$, we have: 


$$
\left\|\left(D f^{n}\right)^{j}\right\|_{0} \leq M^{\prime}|j|
$$

and, for $0<\gamma^{\prime}<1$, since $\left|D f^{n}-1\right|_{\gamma^{\prime}}=\left|D f^{n}\right|_{\gamma^{\prime}}$ :

$$
\left|\left(D f^{n}\right)^{j}\right|_{\gamma^{\prime}} \leq|j| M^{\prime|j|-1}\left|D f^{n}-1\right|_{\gamma^{\prime}}
$$

Therefore, for $0 \leq \gamma^{\prime}<1, \phi \in C^{\gamma^{\prime}}\left(\mathbb{T}^{1}\right)$, we get, by the relations (8) and (9):

$$
\left\|\left(D f^{n}\right)^{j} \phi\right\|_{\gamma^{\prime}} \leq C_{45}\left(\|\phi\|_{\gamma^{\prime}}+\left\|D f^{n}-1\right\|\left\|_{\gamma^{\prime}}\right\| \phi \|_{0}\right)
$$

with $C_{45}(j)=(|j|+1) M^{\prime}|j|$.

Let $\Delta=X_{1}^{j_{1}} \ldots X_{l}^{j_{l}}$ be a monomial of $l$ variables, such that $l=\sum_{p=1}^{l} p j_{p} \geq 1$. Let $0 \leq \gamma^{\prime}<1, n \in \mathbb{Z}$. We estimate $\|\Delta\|_{\gamma^{\prime}}$ when $X_{i}=D^{i} \log D f^{n}$ or when $X_{i}=D^{i+1} f^{n}$, supposing that $l+\gamma^{\prime} \leq k-1$.

The relations (8) and (9) allow estimating $\|\Delta\|_{\gamma^{\prime}}$ by a sum of less than $2^{l}$ terms of the form $\left\|X_{p}\right\|_{\gamma^{\prime}}\left\|\Delta / X_{p}\right\|_{0}, 1 \leq p \leq l, j_{p} \neq 0$. By relation (42), we have:

$$
\begin{aligned}
\left\|D^{p} \log D f^{n}\right\|_{\gamma^{\prime}} & \leq C_{46}\left\|\log D f^{n}\right\|_{l+\gamma^{\prime}}^{\left(p+\gamma^{\prime}\right) /\left(l+\gamma^{\prime}\right)} \\
\left\|D^{p+1} f^{n}\right\|_{\gamma^{\prime}} & \leq C_{47}\left\|D f^{n}-1\right\|_{l+\gamma^{\prime}}^{\left(p+\gamma^{\prime}\right) /\left(l+\gamma^{\prime}\right)} \\
\left\|\frac{\Delta\left(D \log D f^{n}, \ldots, D^{l} \log D f^{n}\right)}{D^{p} \log D f^{n}}\right\|_{0} & \leq C_{48}\left\|\log D f^{n}\right\|_{l+\gamma^{\prime}}^{(l-p) /\left(l+\gamma^{\prime}\right)} \\
\left\|\frac{\Delta\left(D^{2} f^{n}, \ldots, D^{l+1} f^{n}\right)}{D^{p+1} f^{n}}\right\|_{0} & \leq C_{49}\left\|D f^{n}-1\right\|_{l+\gamma^{\prime}}^{(l-p) /\left(l+\gamma^{\prime}\right)}
\end{aligned}
$$

with $C_{46}\left(p, l, \gamma^{\prime}\right)=C_{43}\left(p+\gamma^{\prime}, l+\gamma^{\prime}\right) ; C_{47}\left(p, l, \gamma^{\prime}\right)=C_{44}\left(p+\gamma^{\prime}, l+\gamma^{\prime}\right)$;

$C_{48}\left(p, l, \gamma^{\prime}\right)=\left(C_{43}\left(1, l+\gamma^{\prime}\right)\right)^{l-1}$;

$C_{49}\left(p, l, \gamma^{\prime}\right)=\left(C_{44}\left(1, l+\gamma^{\prime}\right)\right)^{l-1}$

Scheme of the proof. The first two estimates are straightforward. For the third estimate, we write

$\frac{\Delta\left(D \log D f^{n}, \ldots, D^{l} \log D f^{n}\right)}{D^{p} \log D f^{n}}=\left(D \log D f^{n}\right)^{j_{1}} \ldots\left(D^{p} \log D f^{n}\right)^{j_{p}-1} \ldots\left(D^{l} \log D f^{n}\right)^{j_{l}}$

we apply estimation (42) to each $D^{i} \log D f^{n}$ and we use that $\sum j_{k} \leq l$.

The proof of the fourth estimate is analogous, by noting that for $i \geq 1$,

$D^{i}\left(D f^{n}-1\right)=D^{i+1} f^{n}$.

Therefore, when $X_{i}=D^{i} \log D f^{n}$, we get:

$$
\|\Delta\|_{\gamma^{\prime}} \leq C_{50}\left\|\log D f^{n}\right\|_{l+\gamma^{\prime}}
$$

and when $X_{i}=D^{i+1} f^{n}$,

$$
\|\Delta\|_{\gamma^{\prime}} \leq C_{51}\left\|D f^{n}-1\right\|_{l+\gamma^{\prime}}
$$


with $C_{50}(l)=2^{l} \max _{1 \leq p \leq l} \sup _{0 \leq \gamma^{\prime}<1} C_{46}\left(p, l, \gamma^{\prime}\right) C_{48}\left(p, l, \gamma^{\prime}\right)$ and

$C_{51}(l)=2^{l} \max _{1 \leq p \leq l} \sup _{0 \leq \gamma^{\prime}<1} C_{47}\left(p+\gamma, l+\gamma^{\prime}\right) C_{49}\left(p+\gamma^{\prime}, l+\gamma^{\prime}\right)$.

Using appendix 6.4, this allows obtaining the following lemma:

Lemma 5.4. Let $P$ be one of the polynomials of appendix 6.4. $P$ is a polynomial of $l$ variables $X_{1}, \ldots, X_{l}$, homogeneous of weight $l$ if $X_{i}$ has a weight of $i$. For all $n \in \mathbb{Z}$, all $0 \leq \gamma^{\prime}<1$, we have:

$$
\begin{aligned}
\left\|P\left(D \log D f^{n}, \ldots, D^{l} \log D f^{n}\right)\right\|_{\gamma^{\prime}} & \leq C_{52}\left\|\log D f^{n}\right\|_{l+\gamma^{\prime}} \\
\left\|P\left(\frac{D^{2} f^{n}}{D f^{n}}, \ldots, \frac{D^{l+1} f^{n}}{D f^{n}}\right)\right\|_{\gamma^{\prime}} & \leq C_{53}\left\|D f^{n}-1\right\|_{l+\gamma^{\prime}}
\end{aligned}
$$

$$
\begin{aligned}
& \text { with } C_{52}(l)=(4(l+1))^{4(l+1)} C_{50}(l) \text { and } \\
& C_{53}(l)=(4(l+1))^{4(l+1)} C_{45}(-l) C_{51}(l)\left(1+C_{44}\left(l, l+\gamma^{\prime}\right) C_{44}\left(\gamma^{\prime}, l+\gamma^{\prime}\right)\right)
\end{aligned}
$$

Scheme of the proof. The first estimate comes from the preceding discussion. For the second estimate, we write a monomial of $P$ as:

$$
\left(\frac{D^{2} f^{n}}{D f^{n}}\right)^{j_{1}} \ldots\left(\frac{D^{l} f^{n}}{D f^{n}}\right)^{j_{l}}=\left(D^{2} f^{n}\right)^{j_{1}} \ldots\left(D^{l} f^{n}\right)^{j_{l}}\left(D f^{n}\right)^{-\sum j_{k}}
$$

We apply estimate (46), (48), and estimate (43) twice.

Corollary 5.5. For $n \in \mathbb{Z}, 0 \leq \gamma \leq k-1, \gamma=\lfloor\gamma\rfloor+\gamma^{\prime}, 0 \leq \gamma^{\prime}<1$. we have:

$$
C_{54}^{-1}\left\|D f^{n}-1\right\|_{\gamma} \leq\left\|\log D f^{n}\right\|_{\gamma} \leq C_{54}\left\|D f^{n}-1\right\|_{\gamma}
$$

with $C_{54}(\gamma)=M$ if $0 \leq \gamma<1$ and

$$
C_{54}(\gamma)=\left[C_{52}(\lfloor\gamma\rfloor) C_{45}(1)\left(1+M C_{43}(\lfloor\gamma\rfloor \cdot \gamma) C_{43}\left(\gamma^{\prime}, \gamma\right)\right)\right] \vee C_{53}(\lfloor\gamma\rfloor) \text { if } \gamma \geq 1 .
$$

Scheme of the proof. For $0 \leq \gamma<1$, we prove the estimates directly, using that $\log x \leq$ $x-1$.

When $\gamma \geq 1$, for the right-hand side of the estimation, we use formula (77) in appendix 6.4 and the second estimate of lemma 5.4.

For the left-hand side, we apply formula (76) in appendix 6.4, the first estimate of lemma 5.4, relation (46) with $\phi=D^{\lfloor\gamma\rfloor+1} f^{n} / D f^{n}$ and $j=1$, the left-hand side of this estimate of corollary 5.5 with $\gamma<1$, and relation (42) twice.

Using mainly the Faa-d-Bruno formula, we have the lemma [14, p. 350]:

Lemma 5.6. Let $\gamma_{0} \geq \gamma \geq 0, \psi \in D^{\max (1, \gamma)}\left(\mathbb{T}^{1}\right), \phi \in C^{\gamma}\left(\mathbb{T}^{1}\right)$. We have:

$$
\begin{gathered}
\|\phi \circ \psi\|_{\gamma} \leq C_{55}\|\phi\|_{\gamma} \\
\text { with } C_{55}(\gamma, \psi)=\|\psi\|_{\gamma} \text { for } 0 \leq \gamma<1 \text {, and } C_{55}(\gamma, \psi)=((2\lfloor\gamma\rfloor) !)^{2}\|D \psi\|_{\gamma-1}^{L \gamma\rfloor+1} \text { for } \gamma \geq 1 .
\end{gathered}
$$


When $\psi=f^{n}$ for some integer $n$, or when $\psi=h^{-1}$, where $h$ is the conjugacy of $f$ to a rotation, we note:

$$
\begin{aligned}
& C_{55}(\gamma, \psi)=C_{55}(\gamma)=M^{\gamma} \text { if } 0 \leq \gamma<1, \text { and } \\
& C_{55}(\gamma, \psi)=C_{55}(\gamma)=((2\lfloor\gamma\rfloor) !)^{2} M^{\lfloor\gamma\rfloor+1} \text { if } \gamma \geq 1 .
\end{aligned}
$$

Proof. See appendix 6.3.

We have: $f^{n}=h R_{n \alpha} h^{-1}$. We apply lemma 5.6 with $\psi=h^{-1}$ and $\phi=h R_{n \alpha}-h-n \alpha$. To estimate $\left\|h R_{n \alpha}-h-n \alpha\right\|_{\gamma}$, we use the $C^{\gamma}$-norm of $D h$. We get:

Proposition 5.7. For $n \in \mathbb{Z}, 0 \leq \gamma \leq \gamma_{0}$, we have:

$$
\left\|f^{n}-i d-n \alpha\right\|_{\gamma} \leq C_{56}\|n \alpha\|
$$

with $C_{56}(\gamma)=2 M C_{55}(\gamma)$

Let $\alpha_{n}=(-1)^{n}\left(q_{n} \alpha-p_{n}\right)$ and let $\Delta_{s}=\left\|D^{k-1} \log D f^{q_{s}}\right\|_{0}+\alpha_{s}$ (the role of this additional $\alpha_{s}$ is explained at the end of the proof of lemma 5.12). We could also have taken $\left.\Delta_{s}=\left\|D^{k-1} \log D f^{q_{s}}\right\|_{0} \vee \alpha_{s}\right)$. By applying lemma 4.4, and since $M_{s-1} \leq M^{\prime} \alpha_{s-1}$, $1 / m_{s-1} \leq M^{\prime} / \alpha_{s-1}$, and $\alpha_{s-1} \leq 1 / q_{s}$, then we have:

$$
\Delta_{s} \leq\left(C_{27}^{f, k} M^{\prime \frac{3}{2}(k-1)}+1\right) q_{s}^{(k-1) / 2}
$$

Using corollary 5.5, convexity inequalities (proposition 5.3), proposition 5.7, and corollary 5.5 again, we obtain the following lemma:

Lemma 5.8. Let $\gamma \in[0, k-1]$ and $s \geq 0$. We have:

$$
\begin{array}{r}
\left\|\log D f^{q_{s}}\right\|_{\gamma} \leq C_{57}\left(\gamma, \gamma_{0}\right) q_{s+1}^{-1}\left(q_{s+1} \Delta_{s}\right)^{\max \left(0, \frac{\gamma+1-\gamma_{0}}{k-\gamma_{0}}\right)} \\
\text { with } C_{57}\left(\gamma, \gamma_{0}\right)=C_{42}(k) C_{54}(\gamma)^{1+\max \left(0, \frac{\gamma+1-\gamma_{0}}{k-\gamma_{0}}\right)} C_{56}\left(\gamma_{0}\right)^{1-\max \left(0, \frac{\gamma+1-\gamma_{0}}{k-\gamma_{0}}\right)} .
\end{array}
$$

We make a remark on the method and notation: in this lemma 5.8, we estimate the $C^{\gamma}$-norm for $0 \leq \gamma \leq k-1$, instead of only estimating the $C^{\gamma_{1}}$-norm, because of two reasons: first, this lemma is used to obtain lemma 5.9, in which we need an estimation of all the norms of order $\gamma \leq k-1$. Second, in the proof of proposition 5.10, we need an estimate of $\left\|\log D f^{q_{s}}\right\|_{k-1}$.

\subsection{Estimation of $\left\|\log D f^{n q_{s}}\right\|_{\gamma}, 0 \leq n \leq q_{s+1} / q_{s}, 0 \leq \gamma \leq k-1$.}

We use lemma 5.8 to estimate $\left\|\log D f^{n q_{s}}\right\|_{\gamma}, 0 \leq \gamma \leq k-1$ (lemma 5.9) and second, we bootstrap this estimate (lemma 5.12). This bootstrapping allows getting a higher degree of differentiability $\gamma_{1}$ at the end (see estimation (55)).

The Diophantine condition on $\alpha$ implies $q_{s+1} \leq C_{d}^{-1} q_{s}^{1+\beta}$. Therefore, by applying estimation (49), we get:

$$
\left(\Delta_{s} q_{s+1}\right)^{1 / k} q_{s}^{-1} \leq C_{58}(0) q_{s}^{-\epsilon}
$$

With $\epsilon=\frac{1}{2}-\frac{1+2 \beta}{2 k}>0$ and $C_{58}(0)=\left[\left(C_{27}^{f, k} M^{\frac{3}{2}(k-1)}+1\right) C_{d}^{-1}\right]^{1 / k}$

The preceding estimates give the lemma: 
Lemma 5.9. Let $\gamma \in[0, k-1]$. For $s \geq 0,0 \leq n \leq q_{s+1} / q_{s}$, we have:

$$
\left\|\log D f^{n q_{s}}\right\|_{\gamma} \leq C_{59}(\gamma, 0) q_{s}^{-1}\left(q_{s+1} \Delta_{s}\right)^{(\gamma+1) / k}
$$

where, if $0 \leq \gamma<1, C_{59}(\gamma, 0)=C_{57}(\gamma, 0)$, and if $\gamma \geq 1, C_{59}(\gamma, 0)$ is defined inductively by:

$C_{59}(\gamma, 0)=C_{59}(\gamma-1,0)\lfloor\gamma\rfloor^{2} C_{58}(0) C_{52}(\lfloor\gamma\rfloor-1) C_{57}(\gamma, 0) M^{\gamma}\left[2+C_{43}\left(\gamma^{\prime}, \gamma\right) C_{43}(\lfloor\gamma\rfloor, \gamma)\right]$

Scheme of the proof. This lemma is shown by induction on $r=\lfloor\gamma\rfloor$. If $r=0$, we write $\log D f^{n q_{s}}=\sum_{i=0}^{n-1} \log D f^{q_{s}} \circ f^{i}$ and we apply lemma 5.8.

Suppose the lemma holds for $r-1+\gamma^{\prime}$, with $0 \leq \gamma^{\prime}<1$. We have, using the expression (79) in appendix 6.4, and using estimations (8) and (9):

$$
\left\|D^{r} \log D f^{n q_{s}}\right\|_{\gamma^{\prime}} \leq \sum_{l=0}^{r-1} \sum_{i=0}^{n-1}\left(A_{i, l}+B_{i, l}+C_{i, l}\right)
$$

with:

$$
\begin{gathered}
A_{i, l}=\left\|D^{r-l} \log D f^{q_{s}} \circ f^{i q_{s}}\right\|_{\gamma^{\prime}}\left\|\left(D f^{i q_{s}}\right)^{r-l}\right\|_{0}\left\|E_{l}^{r}\right\|_{0} \\
B_{i, l}=\left\|D^{r-l} \log D f^{q_{s}} \circ f^{i q_{s}}\right\|_{0}\left|\left(D f^{i q_{s}}\right)^{r-l}\right|_{\gamma^{\prime}}\left\|E_{l}^{r}\right\|_{0} \\
C_{i, l}=\left\|D^{r-l} \log D f^{q_{s}} \circ f^{i q_{s}}\right\|_{0}\left\|\left(D f^{i q_{s}}\right)^{r-l}\right\|_{0}\left\|E_{l}^{r}\right\|_{\gamma^{\prime}} \\
E_{l}^{r}=E_{l}^{r}\left(D \log D f^{i q_{s}}, \ldots, D^{l} \log D f^{i q_{s}}\right)
\end{gathered}
$$

We estimate $E_{l}^{r}$ with lemma 5.4 (with the polynomial $P=E_{l}^{r}$ ), with (42) (for $B_{i, l}$ ) and with the induction assumption. We estimate $\left\|D^{r-l} \log D f^{q_{s}} \circ f^{i q_{s}}\right\|_{\tilde{\gamma}}, \tilde{\gamma}=0$ or $\gamma^{\prime}$, by applying lemma 5.6 with $\phi=D^{r-l} \log D f^{q_{s}}$ and $\psi=f^{i q_{s}}$, and by applying lemma 5.8 . We estimate $\left|\left(D f^{i q_{s}}\right)^{r-l}\right|_{0}$ with (44). For $\left|\left(D f^{i q_{s}}\right)^{r-l}\right|_{\gamma^{\prime}}$, we apply (45), corollary 5.5, (42) and the induction assumption. We get:

$$
\begin{gathered}
A_{i, l} \leq C_{59}(l, 0) C_{52}(l) C_{57}\left(\gamma^{\prime}+r-l, 0\right) M^{\gamma^{\prime}+r-l} q_{s+1}^{-1} q_{s}^{-1}\left(\Delta_{s} q_{s+1}\right)^{\frac{r-l+\gamma^{\prime}+1}{k}+\frac{l+1}{k}} \\
B_{i, l} \leq C_{57}(r-l, 0) C_{52}(l)(r-l) M^{r-l} C_{43}\left(l, l+\gamma^{\prime}\right) C_{43}\left(\gamma^{\prime}, l+\gamma^{\prime}\right) C_{59}\left(l+\gamma^{\prime}, 0\right) q_{s+1}^{-1} q_{s}^{-1}\left(\Delta_{s} q_{s+1}\right)^{\frac{r-l+1}{k}+\frac{l+\gamma^{\prime}+1}{k}} \\
C_{i, l} \leq M^{r-l} C_{52}(l) C_{57}(r-l, 0) C_{59}\left(l+\gamma^{\prime}, 0\right) q_{s+1}^{-1} q_{s}^{-1}\left(\Delta_{s} q_{s+1}\right)^{\frac{r-l+1}{k}+\frac{l+\gamma^{\prime}+1}{k}}
\end{gathered}
$$

Thus, we have:

$$
A_{i, l}+B_{i, l}+C_{i, l} \leq C_{60}\left(l, \gamma^{\prime}, 0\right) q_{s+1}^{-1} q_{s}^{-1}\left(\Delta_{s} q_{s+1}\right)^{\frac{\gamma+1}{k}+1 / k}
$$

with:

$C_{60}\left(l, \gamma^{\prime}, 0\right)=C_{59}\left(l+\gamma^{\prime}, 0\right) C_{52}(l) C_{57}\left(r-l+\gamma^{\prime}, 0\right) M^{r-l}\left[M^{\gamma^{\prime}}+(r-l) C_{43}\left(\gamma^{\prime}, l+\gamma^{\prime}\right) C_{43}\left(l, l+\gamma^{\prime}\right)+1\right]$

We conclude using estimation (50), and using the fact that the sum (51) has $r n \leq\lfloor\gamma\rfloor q_{s+1} / q_{s}$ terms. 
By applying this lemma 5.9, together with estimate (50), lemma 5.8 and lemma 5.4, we get the proposition [14, p.355]:

Proposition 5.10. The sequence $\left(\Delta_{s} / q_{s}\right)_{s \geq 0}$ is bounded by $C_{61}$.

$C_{61}$ is defined by the following:

$$
\begin{aligned}
& C_{62}=(k-2) M^{k-1} C_{52}(k-2) C_{59}(k-2,0) C_{58}(0) C_{57}(k-1,0) ; \\
& C_{61}=2 C_{27}^{f, k} M^{k-1} \prod_{s=0}^{\infty}\left(1+\frac{5 C_{62}}{q_{s}^{\epsilon}}\right)
\end{aligned}
$$

Proof. We slightly modify Yoccoz's proof. Let $\Delta_{-1}^{\prime}=0$ and, for $s \geq 0$ :

$$
\Delta_{s}^{\prime}=\sup \left\{\left|D^{k-1} \log D f^{q_{t}} \circ f^{m}\left(D f^{m}\right)^{k-1}\right|_{0}, 0 \leq t \leq s, m \geq 0\right\}
$$

For $s \geq 0$, we have: $\Delta_{s} \leq \Delta_{s}^{\prime}+\alpha_{s}$ (This implies $\Delta_{s} \leq C \Delta_{s}^{\prime}$ when $f$ is not a rotation, but contrary to Yoccoz's proof, we do not use this estimate, because the constant $C$ is of the form $C=1+\frac{M^{k-1}}{\left|D^{k-1} \log D f\right|_{0}}$, which diverges as $f$ gets closer to a rotation). We compute a bound on $\left(\Delta_{s}^{\prime}+\alpha_{s}\right) / q_{s}$.

Let $s \geq 0$ (this is another difference with Yoccoz's proof, which only considers $s \geq 1$ ). We have: $q_{s+1}=a_{s+1} q_{s}+q_{s-1}$ (we recall that $q_{-1}=0$ ). Using formula (78) in appendix 6.4 with $g=f^{q_{s-1}}$ and $h=f^{a_{s+1} q_{s}}$, we can write:

$$
\left(D^{k-1} \log D f^{q_{s+1}} \circ f^{m}\right)\left(D f^{m}\right)^{k-1}=X^{\prime}+Y^{\prime}+Z^{\prime}
$$

with:

$$
\begin{gathered}
X^{\prime}=\left(D^{k-1} \log D f^{q_{s-1}} \circ f^{a_{s+1} q_{s}+m}\right)\left(D f^{a_{s+1} q_{s}} \circ f^{m}\right)^{k-1}\left(D f^{m}\right)^{k-1} \\
Y^{\prime}=D^{k-1} \log D f^{a_{s+1} q_{s}} \circ f^{m}\left(D f^{m}\right)^{k-1}
\end{gathered}
$$

$Z^{\prime}=\sum_{l=1}^{k-2}\left(D^{k-1-l} \log D f^{q_{s-1}} \circ f^{a_{s+1} q_{s}+m}\right)\left(D f^{a_{s+1} q_{s}} \circ f^{m}\right)^{k-1-l}\left(D f^{m}\right)^{k-1} G_{l}^{k-1}\left(D \log D f^{a_{s+1} q_{s}} \circ f^{m}, \ldots, D^{l} \log D f^{a_{s+1} q_{s}} \circ f^{m}\right)$

We have:

$$
\left|X^{\prime}\right|_{0} \leq \Delta_{s-1}^{\prime}
$$

Using formula (79) in appendix 6.4 with $g=f^{q_{s}}$, we have:

$Y^{\prime}=\sum_{l=0}^{k-2} \sum_{n=0}^{a_{s+1}-1}\left(D^{k-1-l} \log D f^{q_{s}} \circ f^{n q_{s}+m}\right)\left(D f^{n q_{s}+m}\right)^{k-1-l} E_{l}^{k-1}\left(D \log D f^{n q_{s}}, \ldots, D^{l} \log D f^{n q_{s}}\right) \circ f^{m}\left(D f^{m}\right)^{l}=\sum_{l=0}^{k-2} Y_{l}^{\prime}$

(with the convention $E_{0}^{k-1}=1$ ). We have: $\left|Y_{0}^{\prime}\right|_{0} \leq a_{s+1} \Delta_{s}^{\prime}$.

For $l \geq 1$, we estimate $E_{l}^{k-1}\left(D \log D f^{n q_{s}}, \ldots, D^{l} \log D f^{n q_{s}}\right) \circ f^{m}\left(D f^{m}\right)^{l}$ using lemma 5.6 (with $\psi=f^{m}$ and $\gamma=0$ ), lemma 5.4 (with $P=E_{l}^{k-1}$ ), lemma 5.9 (with $\gamma=l$ ) and estimation (50). We get:

$\left|E_{l}^{k-1}\left(D \log D f^{n q_{s}}, \ldots, D^{l} \log D f^{n q_{s}}\right) \circ f^{m}\left(D f^{m}\right)^{l}\right|_{0} \leq M^{l} C_{52}(l) C_{59}(l, 0) C_{58}(0)\left(\Delta_{s} q_{s+1}\right)^{l / k} q_{s}^{-\epsilon}$ 
By applying lemma 5.8 (with $\gamma=k-1-l$ and $\gamma_{0}=0$ ), and using that $\Delta_{s} \leq \Delta_{s}^{\prime}+\alpha_{s}$, we get:

$$
\left|Y_{l}^{\prime}\right|_{0} \leq a_{s+1}\left(\Delta_{s}^{\prime}+\alpha_{s}\right) M^{k-1} C_{57}(k-1-l, 0) C_{52}(l) C_{59}(l, 0) C_{58}(0) q_{s}^{-\epsilon}
$$

Therefore,

$$
\left|Y^{\prime}\right|_{0} \leq a_{s+1} \Delta_{s}^{\prime}+a_{s+1}\left(\Delta_{s}^{\prime}+\alpha_{s}\right) C_{62} q_{s}^{-\epsilon}
$$

Likewise, we can show that, for $s \geq 1$ :

$$
\left|Z^{\prime}\right|_{0} \leq C_{62} q_{s}^{-\epsilon} q_{s}^{-1}\left(q_{s} \Delta_{s-1}\right)^{\frac{k-l}{k}}\left(q_{s+1} \Delta_{s}\right)^{l / k}
$$

(Yoccoz concludes the estimation of $\left|Z^{\prime}\right|_{0}$ here, using the fact that $q_{s}^{1-l / k} \leq q_{s+1}^{1-l / k}$ and using the fact that $\Delta_{t} \leq C \Delta_{t}^{\prime}, t=s-1, s$. We don't use these facts.)

Since $\Delta_{t} \leq \Delta_{t}^{\prime}+\alpha_{t}, t=s-1, s$, we get:

$$
\begin{gathered}
\left|Z^{\prime}\right|_{0} \leq C_{62} q_{s}^{-\epsilon}\left(\frac{q_{s+1}}{q_{s}}\right)^{l / k}\left(\Delta_{s-1}^{\prime}+\alpha_{s-1}\right)^{1-l / k}\left(\Delta_{s}^{\prime}+\alpha_{s}\right)^{l / k} \\
\left|Z^{\prime}\right|_{0} \leq C_{62} q_{s}^{-\epsilon} \frac{q_{s+1}}{q_{s}}\left(\Delta_{s}^{\prime}+\alpha_{s}\right)\left(\left(1+\frac{\alpha_{s-1}-\alpha_{s}}{\Delta_{s}^{\prime}+\alpha_{s}}\right)\left(\frac{q_{s}}{q_{s+1}}\right)\right)^{1-l / k}
\end{gathered}
$$

Since $\Delta_{s}^{\prime} \geq 0$, and since $a_{s+1} \leq q_{s+1} / q_{s} \leq 2 a_{s+1}$ and $\alpha_{s-1} \leq 2 a_{s+1} \alpha_{s}$, we get:

$$
\left|Z^{\prime}\right|_{0} \leq 4 C_{62} q_{s}^{-\epsilon} a_{s+1}\left(\Delta_{s}^{\prime}+\alpha_{s}\right)
$$

If $s=0, Z^{\prime}=0$. This estimate still holds.

Therefore, for $s \geq 0$,

$$
\alpha_{s+1}+\left|\left(D^{k-1} \log D f^{q_{s+1}} \circ f^{m}\right)\left(D f^{m}\right)^{k-1}\right|_{0} \leq \alpha_{s+1}+\Delta_{s-1}^{\prime}+a_{s+1} \Delta_{s}^{\prime}+a_{s+1}\left(\Delta_{s}^{\prime}+\alpha_{s}\right) 5 C_{62} q_{s}^{-\epsilon}
$$

$\alpha_{s+1}+\left|\left(D^{k-1} \log D f^{q_{s+1}} \circ f^{m}\right)\left(D f^{m}\right)^{k-1}\right|_{0} \leq \alpha_{s+1}-a_{s+1} \alpha_{s}+\Delta_{s-1}^{\prime}+a_{s+1}\left(\Delta_{s}^{\prime}+\alpha_{s}\right)\left(1+5 C_{62} q_{s}^{-\epsilon}\right)$ then

Moreover, we have: $\alpha_{s-1}=a_{s+1} \alpha_{s}+\alpha_{s+1}$. Therefore, for $s \geq 1$, since $\alpha_{s+1}<\frac{1}{2} \alpha_{s-1}$,

$$
\alpha_{s+1}-a_{s+1} \alpha_{s}=2 \alpha_{s+1}-\alpha_{s-1}<0 \leq \alpha_{s-1}
$$

Therefore,

$$
\alpha_{s+1}+\left|\left(D^{k-1} \log D f^{q_{s+1}} \circ f^{m}\right)\left(D f^{m}\right)^{k-1}\right|_{0} \leq \max _{t=s-1, s} \frac{\alpha_{t}+\Delta_{t}^{\prime}}{q_{t}}\left(q_{s-1}+a_{s+1} q_{s}\right)\left(1+5 C_{62} q_{s}^{-\epsilon}\right)
$$

Since $q_{s-1}+a_{s+1} q_{s}=q_{s+1}$, we get:

$$
\frac{\alpha_{s+1}+\left|\left(D^{k-1} \log D f^{q_{s+1}} \circ f^{m}\right)\left(D f^{m}\right)^{k-1}\right|_{0}}{q_{s+1}} \leq \max _{t=s-1, s} \frac{\alpha_{t}+\Delta_{t}^{\prime}}{q_{t}}\left(1+5 C_{62} q_{s}^{-\epsilon}\right)
$$

If $s=0$, we have: 


$$
\frac{\alpha_{1}+\Delta_{1}^{\prime}}{q_{1}} \leq \frac{\alpha_{0}+\Delta_{0}^{\prime}}{q_{0}}\left(1+5 C_{62}\right)
$$

Let $\theta_{s}=\max _{0 \leq t \leq s} \frac{\alpha_{t}+\Delta_{t}^{\prime}}{q_{t}}$. The preceding estimates give:

$$
\theta_{s+1} \leq \theta_{s}\left(1+5 C_{62} q_{s}^{-\epsilon}\right)
$$

Moreover,

$$
\left(\frac{\Delta_{0}^{\prime}+\alpha_{0}}{q_{0}}\right) \leq 1+M^{k-1}\left|D^{k-1} \log D f\right|_{0}
$$

Therefore, for any $s \geq 0$,

$$
\frac{\Delta_{s}}{q_{s}} \leq\left(1+M^{k-1}\left|D^{k-1} \log D f\right|_{0}\right) \prod_{s=0}^{+\infty}\left(1+5 C_{62} q_{s}^{-\epsilon}\right)
$$

To conclude, we apply the claim:

Claim 5.11. Let $C_{27}^{f, k}$ defined in lemma 4.4. For any $k \geq 3$, we have:

$$
\left|D^{k-1} \log D f\right|_{0} \leq \tilde{C}_{27}\left(k,|S f|_{k-3}\right) \leq C_{27}^{f, k}
$$

Proof. First, we recall the observation (see e.g. [14]) that if $x_{0}$ is a point where $(D \log D f)^{2}$ is maximal, then we have:

$$
|S f|_{0} \geq\left|S f\left(x_{0}\right)\right|=\left|D^{2} \log D f\left(x_{0}\right)-\frac{1}{2}\left(D \log D f\left(x_{0}\right)\right)^{2}\right|=\left|\frac{1}{2}\left(D \log D f\left(x_{0}\right)\right)^{2}\right|=\left|\frac{1}{2}(D \log D f)^{2}\right|_{0}
$$

To prove the claim, we proceed by induction on $k$, using the fact that $|D \log D f|_{0} \leq \sqrt{2|S f|_{0}}$ and by applying formula (70) in appendix 6.1. If $k=3$,

$$
\left|D^{2} \log D f\right|_{0} \leq|S f|_{0}+\frac{1}{2}\left|(D \log D f)^{2}\right|_{0} \leq 2|S f|_{0}
$$

Suppose the estimate holds for all $r \leq k$. By formula (70), we have:

$$
D^{k} \log D f=D^{k-2} S f-G_{k}\left(D \log D f, \ldots, D^{k-1} \log D f\right)
$$

As in the proof of lemma 4.4 (see appendix 6.1), we have:

$$
\left|G_{k}\left(D \log D f, \ldots, D^{k-1} \log D f\right)\right| \leq \frac{(2(k-1)) !}{2 k}\left(\tilde{C}_{27}\left(k,|S f|_{k-3}\right)\right)^{k}
$$

We conclude as in the proof of lemma 4.4.

Let us make a remark: by using lemma 5.4, we can improve this estimate. However, lemma 5.4 cannot be used to improve the estimate of lemma 4.4 , because it is a pointwise estimate: an estimate of $\left|D^{k-1} \log D f^{q_{s}}(x)\right|$ in function of $M_{n}^{1 / 2} / m_{n}(x)$. If we only needed an estimate of $\left|D^{k-1} \log D f^{q_{s}}\right|_{0}$ in function of $M_{n}^{1 / 2} / m_{n}$, this improvement would be possible. 
With proposition 5.10, and by using the Diophantine condition $q_{s+1} \leq C_{d}^{-1} q_{s}^{1+\beta}$, we can generalize estimation (50) and lemma 5.9, for $\gamma_{0}>0$. The generalization of estimation (50) is:

$$
\left(\Delta_{s} q_{s+1}\right)^{1 /\left(k-\gamma_{0}\right)} q_{s}^{-1} \leq C_{58}\left(\gamma_{0}\right) q_{s}^{\frac{\beta+2}{k-\gamma_{0}}-1}
$$

with $C_{58}\left(\gamma_{0}\right)=C_{61}^{\frac{1}{k-\gamma_{0}}} C_{d}^{\frac{-1}{k-\gamma_{0}}}$.

The generalization of lemma 5.9 is:

Lemma 5.12. Let $\gamma \in[0, k-1]$. For $s \geq 0,0 \leq n \leq q_{s+1} / q_{s}$, we have:

$$
\left\|\log D f^{n q_{s}}\right\|_{\gamma} \leq C_{59}\left(\gamma, \gamma_{0}\right) q_{s}^{-1}\left(q_{s+1} \Delta_{s}\right)^{\frac{\gamma+1-\gamma_{0}}{k-\gamma_{0}} \vee 0}
$$

where, if $0 \leq \gamma<1, C_{59}\left(\gamma, \gamma_{0}\right)=\max \left(C_{54}(\gamma) C_{56}(\gamma+1), C_{57}\left(\gamma, \gamma_{0}\right)\right)$, and if $\gamma \geq 1$, $C_{59}(\gamma, 0)$ is defined inductively by:

$$
C_{59}\left(\gamma, \gamma_{0}\right)=C_{59}\left(\gamma-1, \gamma_{0}\right)\lfloor\gamma\rfloor C_{58}\left(\gamma_{0}\right) C_{52}(\lfloor\gamma\rfloor-1)
$$

$C_{59}\left(\gamma, \gamma_{0}\right)=2^{1+0 \vee \frac{\gamma+1-\gamma_{0}}{k-\gamma_{0}}} C_{59}\left(\gamma-1, \gamma_{0}\right)\lfloor\gamma\rfloor^{2} C_{58}\left(\gamma_{0}\right) C_{52}(\lfloor\gamma\rfloor-1) C_{57}\left(\gamma, \gamma_{0}\right) M^{\gamma}\left[2+C_{43}\left(\gamma^{\prime}, \gamma\right) C_{43}(\lfloor\gamma\rfloor, \gamma)\right]$

Remark 5.13. When $\gamma \geq 1$, the definitions of $C_{59}\left(\gamma, \gamma_{0}\right)$ are analogous to those of $C_{59}(\gamma, 0)$ given in lemma 5.9 , by replacing 0 with $\gamma_{0}$, by a factor $2^{1+0 \vee \frac{\gamma+1-\gamma_{0}}{k-\gamma_{0}}}$.

Scheme of the proof. We give the scheme of the proof in order to explain the additional $\alpha_{s}$ in the definition of $\Delta_{s}$ (this additional $\alpha_{s}$ makes necessary our modification of Yoccoz's proof of proposition 5.10).

If $\gamma_{0}-1 \leq \gamma<1$, we proceed as in lemma 5.9. If $\gamma \leq \gamma_{0}-1$ and $\gamma<1$, we apply corollary 5.5 and proposition 5.7. The induction step is analogous to the proof of lemma 5.9, except the end: indeed, by proceeding as in lemma 5.9, we have:

$$
\begin{gathered}
A_{i, l} \leq C_{59}\left(l, \gamma_{0}\right) C_{52}(l) C_{57}\left(\gamma^{\prime}+r-l, \gamma_{0}\right) M^{\gamma^{\prime}+r-l} q_{s+1}^{-1} q_{s}^{-1}\left(\Delta_{s} q_{s+1}\right)^{0 \vee \frac{r-l+\gamma^{\prime}+1-\gamma_{0}}{k-\gamma_{0}}+0 \vee \frac{l+1-\gamma_{0}}{k-\gamma_{0}}} \\
B_{i, l} \leq C_{57}(r-l, 0) C_{52}(l)(r-l) M^{r-l} C_{43}\left(l, l+\gamma^{\prime}\right) C_{43}\left(\gamma^{\prime}, l+\gamma^{\prime}\right) C_{59}\left(l+\gamma^{\prime}, 0\right) q_{s+1}^{-1} q_{s}^{-1}\left(\Delta_{s} q_{s+1}\right)^{0 \vee \frac{r-l+1-\gamma_{0}}{k-\gamma_{0}}+0 \vee \frac{l+\gamma^{\prime}+1-\gamma_{0}}{k-\gamma_{0}}} \\
C_{i, l} \leq M^{r-l} C_{52}(l) C_{57}(r-l, 0) C_{59}\left(l+\gamma^{\prime}, 0\right) q_{s+1}^{-1} q_{s}^{-1}\left(\Delta_{s} q_{s+1}\right)^{0 \vee \frac{r-l+1-\gamma_{0}}{k-\gamma_{0}}+0 \vee \frac{l+\gamma^{\prime}+1-\gamma_{0}}{k-\gamma_{0}}}
\end{gathered}
$$

We have:

$\left(0 \vee \frac{r-l+1-\gamma_{0}}{k-\gamma_{0}}+0 \vee \frac{l+\gamma^{\prime}+1-\gamma_{0}}{k-\gamma_{0}}\right) \vee\left(0 \vee \frac{r-l+\gamma^{\prime}+1-\gamma_{0}}{k-\gamma_{0}}+0 \vee \frac{l+1-\gamma_{0}}{k-\gamma_{0}}\right) \leq 0 \vee \frac{\gamma+1-\gamma_{0}}{k-\gamma_{0}}+\frac{1}{k-\gamma_{0}}$

Moreover, since $2 q_{s+1} \Delta_{s} \geq 2 q_{s+1} \alpha_{s} \geq 1$, then

$$
A_{i, l}+B_{i, l}+C_{i, l} \leq 2^{\frac{1}{k-\gamma_{0}}+0 \vee \frac{\gamma+1-\gamma_{0}}{k-\gamma_{0}}} C_{60}\left(l, \gamma^{\prime}, \gamma_{0}\right) q_{s+1}^{-1} q_{s}^{-1}\left(\Delta_{s} q_{s+1}\right)^{0 \vee \frac{\gamma+1-\gamma_{0}}{k-\gamma_{0}}+\frac{1}{k-\gamma_{0}}}
$$

with:

$$
C_{60}\left(l, \gamma^{\prime}, \gamma_{0}\right)=C_{59}\left(l+\gamma^{\prime}, \gamma_{0}\right) C_{52}(l) C_{57}\left(r-l+\gamma^{\prime}, \gamma_{0}\right) M^{r-l}\left[M^{\gamma^{\prime}}+(r-l) C_{43}\left(\gamma^{\prime}, l+\gamma^{\prime}\right) C_{43}\left(l, l+\gamma^{\prime}\right)+1\right]
$$

(this is why we define $\Delta_{s}=\left|D^{k-1} \log D f^{q_{s}}\right|_{0}+\alpha_{s}$. If we defined $\Delta_{s}=\left|D^{k-1} \log D f^{q_{s}}\right|_{0}$ and if $\left|D^{k-1} \log D f^{q_{s}}\right|_{0}$ was too small, we could not do this estimate). 
By using estimation (53) and lemma 5.12, we obtain, for $0 \leq n \leq\left(q_{s+1}\right) / q_{s}$, and $0 \leq \gamma \leq \gamma_{1}[14$, p.357]:

$$
\left\|\log D f^{n q_{s}}\right\|_{\gamma} \leq C_{63}\left(\gamma, \gamma_{0}\right) q_{s}^{\rho\left(\gamma, \gamma_{0}\right)}
$$

with

$$
\rho\left(\gamma, \gamma_{0}\right)=\frac{(2+\beta)\left(0 \vee\left(\gamma+1-\gamma_{0}\right)\right)}{k-\gamma_{0}}-1
$$

and

$$
C_{63}\left(\gamma, \gamma_{0}\right)=C_{59}\left(\gamma, \gamma_{0}\right)\left(C_{d}^{-1} C_{61}\right)^{\frac{\gamma+1-\gamma_{0}}{k-\gamma_{0}} \vee 0}
$$

Notice that for any

$$
\gamma_{1}<g\left(\gamma_{0}\right)=\frac{(1+\beta) \gamma_{0}+k-(2+\beta)}{2+\beta}
$$

we have $\rho\left(\gamma_{1}, \gamma_{0}\right)<0$ (we will take $\left.\gamma_{1}=\frac{1}{2}\left(g\left(\gamma_{0}\right)+\gamma_{0}\right)\right)$.

This implies $\sum_{s \geq 0} q_{s}^{\rho\left(\gamma_{1}, \gamma_{0}\right)}<+\infty$, which will allow estimating $\left\|\log D f^{N}\right\|_{\gamma_{1}}$, as we will see in the next subsection.

A remark on the method and notation: we establish estimate (54) for any $0 \leq \gamma \leq \gamma_{1}$ (and not just for $\gamma_{1}$ ) because we need it for the estimate of the quantity $Z$ defined below.

\subsection{Estimation of $\left\|\log D f^{N}\right\|_{\gamma_{1}}$}

Proposition 5.14. Let $N$ be an integer and let us write $\gamma_{1}=r+\gamma_{1}^{\prime}$, with $0 \leq \gamma_{1}^{\prime}<1$ and $r$ integer. We have:

$$
\left\|\log D f^{N}\right\|_{\gamma_{1}} \leq C_{63}\left(\gamma_{1}, \gamma_{0}\right) \prod_{s=1}^{\infty}\left(1+\frac{C_{64}\left(\gamma_{1}, \gamma_{0}\right)+C_{66}\left(\gamma_{1}, \gamma_{0}\right)}{q_{s}^{-\rho\left(\gamma_{1}, \gamma_{0}\right)}}\right)=C_{67}
$$

with:

$$
\begin{gathered}
C_{64}\left(\gamma_{1}, \gamma_{0}\right)=C_{45}(r) C_{55}\left(\gamma_{1}^{\prime}\right) C_{63}\left(\gamma_{1}, \gamma_{0}\right) C_{54}\left(\gamma_{1}^{\prime}\right) \\
C_{66}\left(\gamma_{1}, \gamma_{0}\right)=(r-1) C_{64}\left(\gamma_{1}, \gamma_{0}\right) C_{52}(r-1)\left(2+C_{43}\left(\gamma_{1}^{\prime}, \gamma_{1}\right) C_{43}\left(r, \gamma_{1}\right)\right)
\end{gathered}
$$

Scheme of the proof. We write $N=\sum_{s=0}^{S} b_{s} q_{s}$ with $0 \leq b_{s} \leq \frac{q_{s+1}}{q_{s}}$ and $b_{s}$ integer. Let $N_{s}=\sum_{t=0}^{s} b_{t} q_{t}$ for $0 \leq s \leq S$. Moreover, let us write $\gamma_{1}=r+\gamma_{1}^{\prime}$, with $0 \leq \gamma_{1}^{\prime}<1$ and $r$ integer. By formula (78) in appendix 6.4, we can write $D^{r} \log D f^{N_{s}}=X+Y+Z$ with:

$$
\begin{aligned}
X & =\left(D^{r} \log D f^{b_{s} q_{s}} \circ f^{N_{s-1}}\right)\left(D f^{N_{s-1}}\right)^{r} \\
Y & =D^{r} \log D f^{N_{s-1}} \\
Z & =\sum_{l=1}^{r-1}\left(D^{r-l} \log D f^{b_{s} q_{s}} \circ f^{N_{s-1}}\right)\left(D f^{N_{s-1}}\right)^{r-l} G_{l}^{r} \\
G_{l}^{r} & =G_{l}^{r}\left(D \log D f^{N_{s-1}}, \ldots, D^{l} \log D f^{N_{s-1}}\right)
\end{aligned}
$$


We successively estimate $X$ and $Z$. For $X$, we use estimate (46), corollary 5.5 and lemma 5.6 with $\phi=D^{r} \log D f^{b_{s} q_{s}}$ and $\psi=f^{N_{s-1}}$. We also use estimate (54), and the fact that $q_{s}^{\rho\left(r, \gamma_{0}\right)} \leq q_{s}^{\rho\left(\gamma_{1}, \gamma_{0}\right)}$. We get:

$$
\|X\|_{\gamma_{1}^{\prime}} \leq C_{64} q_{s}^{\rho\left(\gamma_{1}, \gamma_{0}\right)}\left(1+\left\|\log D f^{N_{s-1}}\right\|_{\gamma_{1}^{\prime}}\right)
$$

with

$$
C_{64}\left(\gamma_{1}, \gamma_{0}\right)=C_{45}(r) C_{55}\left(\gamma_{1}^{\prime}\right) C_{63}\left(\gamma_{1}, \gamma_{0}\right) C_{54}\left(\gamma_{1}^{\prime}\right)
$$

We estimate $Z$. By applying estimation (9), we have:

$\|Z\|_{\gamma_{1}^{\prime}} \leq \sum_{l=1}^{r-1}\left|\left(D^{r-l} \log D f^{b_{s} q_{s}} \circ f^{N_{s-1}}\right)\left(D f^{N_{s-1}}\right)^{r-l}\right|_{0}\left|G_{l}^{r}\right|_{\gamma_{1}^{\prime}}+\left\|\left(D^{r-l} \log D f^{b_{s} q_{s}} \circ f^{N_{s-1}}\right)\left(D f^{N_{s-1}}\right)^{r-l}\right\|_{\gamma_{1}^{\prime}}\left|G_{l}^{r}\right|_{0}$

As with $X$, we have:

$$
\left\|\left(D^{r-l} \log D f^{b_{s} q_{s}} \circ f^{N_{s-1}}\right)\left(D f^{N_{s-1}}\right)^{r-l}\right\|_{\gamma_{1}^{\prime}} \leq C_{65} q_{s}^{\rho\left(\gamma_{1}, \gamma_{0}\right)}\left(1+\left\|\log D f^{N_{s-1}}\right\|_{\gamma_{1}^{\prime}}\right)
$$

with:

$$
C_{65}\left(\gamma_{1}, \gamma_{0}, l\right)=C_{45}(r-l) C_{55}\left(\gamma_{1}^{\prime}\right) C_{63}\left(\gamma_{1}^{\prime}+r-l, \gamma_{0}\right) C_{54}\left(\gamma_{1}^{\prime}\right) \leq C_{64}\left(\gamma_{1}, \gamma_{0}\right)
$$

Moreover, by estimate (54), we also have:

$$
\left|\left(D^{r-l} \log D f^{b_{s} q_{s}} \circ f^{N_{s-1}}\right)\left(D f^{N_{s-1}}\right)^{r-l}\right|_{0} \leq M^{r-l} C_{63}\left(r-l, \gamma_{0}\right) q_{s}^{\rho\left(r-l, \gamma_{0}\right)} \leq C_{64} q_{s}^{\rho\left(\gamma_{1}, \gamma_{0}\right)}
$$

For $G_{l}^{r}$, we use lemma 5.4 with the polynomial $P=G_{l}^{r}$ (see appendix 6.4). We estimate $\|Z\|_{\gamma_{1}^{\prime}}$ by applying estimation (42) twice. We get:

$$
\|Z\|_{\gamma_{1}^{\prime}} \leq C_{66} q_{s}^{\rho\left(\gamma_{1}, \gamma_{0}\right)}\left\|\log D f^{N_{s-1}}\right\|_{\gamma_{1}}
$$

with:

$$
C_{66}\left(\gamma_{1}, \gamma_{0}\right)=(r-1) C_{64}\left(\gamma_{1}, \gamma_{0}\right) C_{52}(r-1)\left(2+C_{43}\left(\gamma_{1}^{\prime}, \gamma_{1}\right) C_{43}\left(r, \gamma_{1}\right)\right)
$$

Therefore, since $\|Y\|_{\gamma_{1}^{\prime}}=\left\|D^{r} \log D f^{N_{s-1}}\right\|_{\gamma_{1}^{\prime}}$, we get, for $s \geq 1$ :

$$
\left\|D^{r} \log D f^{N_{s}}\right\|_{\gamma_{1}^{\prime}} \leq\left\|\log D f^{N_{s}}\right\|_{\gamma_{1}} \leq\left(1+\frac{C_{64}\left(\gamma_{1}, \gamma_{0}\right)+C_{66}\left(\gamma_{1}, \gamma_{0}\right)}{q_{s}^{-\rho\left(\gamma_{1}, \gamma_{0}\right)}}\right)\left\|D^{r} \log D f^{N_{s-1}}\right\|_{\gamma_{1}^{\prime}}
$$

Moreover, by estimate (54), since $N_{0}=b_{0}$, we also have:

$$
\left\|D^{r} \log D f^{N_{0}}\right\|_{\gamma_{1}^{\prime}} \leq C_{63}\left(\gamma_{1}, \gamma_{0}\right)
$$

We conclude that:

$$
\left\|\log D f^{N}\right\|_{\gamma_{1}} \leq C_{63}\left(\gamma_{1}, \gamma_{0}\right) \prod_{s=1}^{\infty}\left(1+\frac{C_{64}\left(\gamma_{1}, \gamma_{0}\right)+C_{66}\left(\gamma_{1}, \gamma_{0}\right)}{q_{s}^{-\rho\left(\gamma_{1}, \gamma_{0}\right)}}\right)=C_{67}
$$




\subsubsection{Computation of the estimations: proof of proposition 5.1.}

The quantity $C_{67}$ depends on $\sup _{p \geq 0}\left\|\log D f^{p}\right\|_{\gamma_{0}}$. We estimate $C_{67}$. First, we estimate $C_{61}$. Since $5 C_{62} \leq\left(C_{59}(k-1,0)\right)^{2}$, we estimate $C_{59}(\gamma, 0)$ for $0 \leq \gamma \leq k-1$. By combining the constants appearing in lemma 5.9, we get:

$$
C_{59}(\gamma, 0) \leq C_{59}\left(\gamma^{\prime}, 0\right)\left[(k-1) 2 C_{58}(0) C_{57}(k-1,0) C_{52}(k-1) M^{k}\left(\log M C_{42}(k)\right)^{2}\right]^{k-1}
$$

Moreover,

$$
\begin{gathered}
C_{52}(k-1) \leq(4 k)^{4 k} 2^{k-1}\left(C_{42}(k) \log M\right)^{k-1} \\
C_{53}(k-1) \leq(4 k)^{4 k} k M^{k-1} 2^{k-1}\left(C_{42}(k)(M+1)\right)^{k-1}\left(1+\left((M+1) C_{42}(k)\right)^{2}\right) \\
C_{53}(k-1) \leq(4 k)^{4 k} k 2^{2 k+1} M^{2 k}\left(C_{42}(k)\right)^{k+1}
\end{gathered}
$$

Note that for any $l \geq 2, C_{54}(l)=C_{53}(l)$. Since

$$
C_{57}(k-1,0) \leq\left[C_{54}(k-1)\right]^{2} 2 M C_{42}(k)
$$

We get:

$$
C_{57}(k-1,0) \leq(4 k)^{8 k} k^{2} 2^{4 k+3}\left(C_{42}(k)\right)^{2 k+3} M^{4 k+1}=\tilde{C}_{57}(k-1,0)
$$

We have:

$$
C_{59}(\gamma, 0) \leq C_{59}\left(\gamma^{\prime}, 0\right)\left[C_{58}(0)\left(\tilde{C}_{57}(k-1,0)\right)^{2} M^{k}\right]^{k-1}
$$

Since $C_{58}(0) \leq\left(2 M^{\frac{3}{2}(k-1)} C_{27}^{f, k} C_{d}^{-1}\right)^{1 / k}$, we get, for $0 \leq \gamma \leq k-1$ :

$$
C_{59}(\gamma, 0) \leq C_{d}^{-1} C_{27}^{f, k} 2 M^{k^{2}+\frac{3}{2}(k-1)}\left[\tilde{C}_{57}(k-1,0)\right]^{2 k}=\tilde{C}_{59}
$$

We get:

$$
C_{d}^{-1} C_{61} \leq C_{d}^{-1} C_{27}^{f, k} M^{k-1} \prod_{s=0}^{\infty}\left(1+\frac{\left(\tilde{C}_{59}\right)^{2}}{q_{s}^{\epsilon}}\right) \leq \prod_{s=0}^{\infty}\left(1+\frac{2 C_{d}^{-1} C_{27}^{f, k} M^{k-1}\left(\tilde{C}_{59}\right)^{2}}{q_{s}^{\epsilon}}\right)
$$

We estimate $C_{64}\left(\gamma_{1}, \gamma_{0}\right)$ and $C_{66}\left(\gamma_{1}, \gamma_{0}\right)$.

We have: $C_{64}\left(\gamma_{1}, \gamma_{0}\right) \leq 2(r+1) M^{r+1} C_{63}\left(\gamma_{1}, \gamma_{0}\right) C_{55}\left(\gamma_{1}^{\prime}\right)$.

Therefore,

$$
C_{64}\left(\gamma_{1}, \gamma_{0}\right) \leq\left(\max _{0 \leq \gamma \leq k-1} C_{59}\left(\gamma, \gamma_{0}\right)\right)\left(C_{d}^{-1} C_{61}\right)^{\frac{k-1}{k-\gamma_{0}}} C_{55}\left(\gamma_{1}^{\prime}\right) 2(r+1) M^{r+1}
$$

We estimate $C_{66}\left(\gamma_{1}, \gamma_{0}\right)$. We have:

$$
C_{66}\left(\gamma_{1}, \gamma_{0}\right) \leq(r-1)\left(2+\left((\log M) C_{42}(k)\right)\right)^{2} C_{64}\left(\gamma_{1}, \gamma_{0}\right) C_{52}(r-1)
$$

To complete the estimations of $C_{64}\left(\gamma_{1}, \gamma_{0}\right)$ and $C_{66}\left(\gamma_{1}, \gamma_{0}\right)$, we need to estimate $C_{59}\left(\gamma, \gamma_{0}\right)$. By writing $\gamma=\lfloor\gamma\rfloor+\gamma^{\prime}$, and by proceeding as for the estimation of $C_{59}(\gamma, 0)$, we have: 


$$
C_{59}\left(\gamma, \gamma_{0}\right) \leq C_{59}\left(\gamma^{\prime}, \gamma_{0}\right)\left(C_{d}^{-1} C_{61}\right)^{\frac{k-1}{k-\gamma_{0}}} C_{57}\left(k-1, \gamma_{0}\right)^{2 k} M^{k^{2}} 2^{2(k-1)}
$$

Moreover,

$$
C_{57}\left(k-1, \gamma_{0}\right) \leq C_{57}(k-1,0) C_{55}(k-1)
$$

We can also check that:

$$
C_{59}\left(\gamma^{\prime}, \gamma_{0}\right)=2 C_{42}(k) M^{3 \gamma^{\prime}+1} \vee 2 M^{\gamma^{\prime}+1} C_{55}\left(\gamma^{\prime}+1\right) \leq \tilde{C}_{57}(k-1,0) C_{55}(k-1)
$$

Therefore,

$$
C_{64}\left(\gamma_{1}, \gamma_{0}\right) \leq\left(C_{d}^{-1} C_{61}\right)^{\frac{2(k-1)}{k-\gamma_{0}}}\left(\tilde{C}_{57}(k-1,0)\right)^{2 k+1}\left(C_{55}(k-1)\right)^{2 k+2} 2 k M^{k^{2}+k}
$$

Now, let

$$
\tau_{1}=\frac{5(k-1)}{\beta+2+\eta}
$$

let $C_{68}=(k+2)^{400 k^{4}} M^{24 k(k+1)}\left(C_{27}^{f, k}\right)^{4 k}\left(C_{d}^{-1}\right)^{4 k^{2}}$. Let also

$$
\epsilon_{1}=\min \left(\epsilon, \frac{\eta}{2(\beta+2+\eta)}\right)
$$

We have: $\epsilon_{1} \leq \min \left(\epsilon,-\rho\left(\gamma_{1}, \gamma_{0}\right)\right)$ and for any $\gamma_{0} \leq k-2-\beta-\eta$, we have $\tau_{1} \geq \frac{2(k-1)}{k-\gamma_{0}}$.

Note that $C_{42}(k)$ only depends on $k$, and that $\tilde{C}_{57}(k-1,0)$ and $C_{55}(k-1)$ only depend on $k$ and $M$.

By combining estimations (57), (58), (59), (61) and (60), we can check that we have:

$$
C_{67} \leq \prod_{s=0}^{\infty}\left(1+\frac{C_{68} \prod_{s=0}^{\infty}\left(1+\frac{C_{68}}{q_{s}^{\epsilon_{1}}}\right)^{\tau_{1}}}{q_{s}^{\epsilon_{1}}}\right)
$$

Since $q_{s} \geq(\sqrt{2})^{s-1}$, we get:

$$
C_{67} \leq \prod_{s \geq 0}\left(1+\frac{\sqrt{2} C_{68} \prod_{s \geq 0}\left(1+\frac{\sqrt{2} C_{68}}{2^{\epsilon_{1}}}\right)^{\tau_{1}}}{2^{s \frac{\epsilon_{1}}{2}}}\right)
$$

In order to obtain the final estimation, we need the claim:

Claim 5.15. Let $C \geq 10$. For any $2 \geq u>1$, we have:

$$
\prod_{n=0}^{\infty}\left(1+\frac{C}{u^{n}}\right) \leq e^{\frac{2^{2 / 3}}{\log u}(\log C)^{2}}
$$


Proof.

$$
\begin{array}{r}
\sum_{n \geq 0} \log \left(1+C / u^{n}\right)=\sum_{n \leq \frac{\log C}{\log u}-1} \log \left(1+C / u^{n}\right)+\sum_{n>\frac{\log C}{\log u}-1} \log \left(1+C / u^{n}\right) \\
\leq \frac{\log C}{\log u} \log (1+C)+\sum_{n>\frac{\log C}{\log u}-1} C / u^{n} \\
\leq \frac{\log C}{\log u}(\log (1+C)+1) \leq \frac{2^{2 / 3}}{\log u}(\log C)^{2}
\end{array}
$$

for $C \geq 10$.

By applying this proposition twice, we get the claim:

Claim 5.16. Let $C \geq 10,2 \geq u>1, \tau \geq 1$. We have:

$$
\prod_{n \geq 0}\left(1+\frac{C \prod_{n=0}^{\infty}\left(1+\frac{C}{u^{n}}\right)^{\tau}}{u^{n}}\right) \leq e \wedge\left(\frac{18 \tau^{2}}{(\log u)^{3}}(\log C)^{4}\right)
$$

Let $C_{69}=\sqrt{2} C_{68}$. We apply claim 5.16 with $C=C_{69}, u=2^{\frac{\epsilon_{1}}{2}}, \tau=\tau_{1}$. We obtain:

$$
C_{67} \leq e \wedge\left(\frac{18 \tau_{1}^{2}}{\left(\frac{\epsilon_{1}}{2} \log 2\right)^{3}}\left(\log C_{69}\right)^{4}\right)
$$

Moreover, let

$$
C_{70}=\frac{18 \tau_{1}^{2}}{\left(\frac{\epsilon_{1}}{2} \log 2\right)^{3}}
$$

and let $C_{71}^{-}=\frac{18 \times 5^{2} \times 8}{(\log 2)^{3}}$ a numerical constant. We have:

$$
C_{70} \leq C_{71}^{-} \frac{(k-1)^{2}}{\epsilon_{1}^{2}}
$$

By using the definitions of $\epsilon_{1}$ (see (63)) and $\tau_{1}$ (see (62)), since $\epsilon=\frac{k-(1+2 \beta)}{2 k}$ and since $\eta \leq k-2-\beta$, we have:

$C_{70} \leq C_{71}^{-} \frac{k^{2}}{\left(\min \left(\frac{k-(2 \beta+1)}{2 k}, \frac{\eta}{2(\beta+2+\eta)}\right)\right)^{3}} \leq 8 C_{71}^{-} \frac{k^{5}}{(\min (k-(2 \beta+1), k-(\beta+2)))^{3}}=C_{72}(k, \beta)$

Therefore, we get:

$$
\left\|\log D f^{N}\right\|_{\gamma_{1}} \leq e \wedge\left(C_{72}(k, \beta)\left(C_{73}^{f, k}+4 k^{2} \log \left(C_{d}^{-1}\right)+24 k(k+1) \sup _{p \geq 0}\left\|\log D f^{p}\right\|_{\gamma_{0}}\right)^{4}\right)
$$

with:

$$
C_{73}^{f, k}=\log \left(\sqrt{2}(k+2)^{400 k^{4}}\left(C_{27}^{f, k}\right)^{4 k}\right)
$$

Hence proposition 5.1 . 


\subsubsection{Proof of theorem 1.6: estimations (6) and (7).}

By corollary 5.5, we have:

$$
\left\|D f^{N}\right\|_{\frac{k}{2(\beta+2)}-\frac{1}{2}} \leq C_{54}(k-1)\left(1+\left\|\log D f^{N}\right\|_{\frac{k}{2(\beta+2)}-\frac{1}{2}}\right)
$$

Moreover, we recall that:

$$
C_{54}(k-1) \leq(4 k)^{4 k} k 2^{2 k+1} M^{2 k}\left(C_{42}(k)\right)^{k+1}
$$

We have: $C_{54}(k-1) \leq C_{74}(k) M^{2 k}$ with $C_{74}(k)=(4 k)^{4 k} k 2^{(k+1)^{3}+3 k+2}$

Moreover, $\|D h\|_{\frac{k}{2(\beta+2)}-\frac{1}{2}} \leq \sup _{N \geq 0}\left\|D f^{N}\right\|_{\frac{k}{2(\beta+2)}-\frac{1}{2}}$. We get:

$\|D h\|_{\frac{k}{2(\beta+2)}-\frac{1}{2}} \leq e \wedge\left(C_{72}(k, \beta)\left(C_{73}^{f, k}+4 k^{2} \log C_{d}^{-1}+24 k(k+1) \log M^{\prime}\right)^{4}+\log \left(C_{74}(k)\right)+2 k \log M^{\prime}\right)$

Since $2 k \log M^{\prime} \leq k(k+1) \log M^{\prime}$, we get:

$$
\|D h\|_{\frac{k}{2(\beta+2)}-\frac{1}{2}} \leq e \wedge\left(C_{72}(k, \beta)\left(C_{73}^{f, k}+4 k^{2} \log C_{d}^{-1}+25 k(k+1) \log M^{\prime}+\log \left(C_{74}(k)\right)\right)^{4}\right)
$$

We show estimation (6). We suppose $k \geq 3 \beta+9 / 2$. Let:

$C_{75}\left(\beta, C_{d},|D f|_{0}, W(f),|S f|_{0},|S f|_{k-3}\right)=e^{(3)} \wedge\left(C_{3}(\beta) C_{4}\left(C_{d}\right) C_{5}\left(|D f|_{0}, W(f),|S f|_{0}\right) C_{6}\left(|S f|_{k-3}\right)\right)$

i.e. we consider the bound given by estimation (2), except that we replace $|S f|_{[3 \beta+3 / 2]}$ with $|S f|_{k-3}$.

$C_{27}^{f, k}$ depends on $k,|S f|_{k-3}$ and $W(f)$. We have:

$4 k \log C_{27}\left(k,|S f|_{k-3}, W(f)\right) \leq 4 k(k+4) ! \log C_{27}\left(3 \beta+9 / 2,|S f|_{k-3}, W(f)\right) \leq 4(k+5) ! \log C_{75}$

Moreover, since $M^{\prime} \leq C_{75}$ and $k \geq 5$,

$4 k^{2} \log C_{d}^{-1}+25 k(k+1) \log M^{\prime}+\log \left(C_{74}(k)\right)+400 k^{4} \log (\sqrt{2}(k+2)) \leq C_{76}(k) \log C_{75}$

with $C_{76}=4 k^{2}+25 k(k+1)+\log \left(C_{74}(k)\right)+400 k^{4} \log (\sqrt{2}(k+2)) \leq(k+5) !$. Therefore,

$$
\|D h\|_{\frac{k}{2(\beta+2)}-\frac{1}{2}} \leq e \wedge\left(C_{72}(k, \beta)\left(5(k+5) ! \log C_{75}\right)^{4}\right)
$$

We also have:

$$
C_{72}(k, \beta) \leq 8 C_{71}^{-} \frac{k^{5}}{(\min (k-(2 \beta+1), k-(\beta+2)))^{3}} \leq C_{71}^{-} k^{5}
$$

Since $C_{71}^{-} k^{5}(5(k+5) !)^{4} \leq C_{71}^{-} 5^{4}((k+7) !)^{4}$, and since $2 \geq 2 \log 2$, we conclude:

$$
\|D h\|_{\frac{k}{2(\beta+2)}-\frac{1}{2}} \leq e \wedge\left(C_{12}(k) e^{(2)} \wedge\left(2+C_{3}(\beta) C_{4}\left(C_{d}\right) C_{5}\left(|D f|_{0}, W(f),|S f|_{0}\right) C_{6}\left(|S f|_{k-3}\right)\right)\right)
$$

with:

$$
C_{12}(k)=\frac{18 \times 5^{6} \times 8}{(\log 2)^{3}}((k+7) !)^{4}
$$


If $\beta=0$, we can use the $C^{1}$ estimate. We have: $\log M^{\prime} \leq C_{1} / C_{d}$ and therefore,

$$
4 k^{2} \log \left(C_{d}^{-1}\right)+25 k(k+1) \log M^{\prime} \leq(k+5) ! C_{1} / C_{d}
$$

Moreover,

$$
C_{73}^{f, k}+\log \left(C_{74}(k)\right) \leq 4(k+5) !\left(\log \left(1 \vee|S f|_{k-3}\right)+W(f)+1\right)
$$

then by using estimation (67), we obtain:

$$
\|D h\|_{\frac{k}{4}-\frac{1}{2}} \leq e \wedge\left(C_{13}(k)\left[C_{14}\left[W(f),|S f|_{k-3}\right]+\frac{C_{1}\left[W(f),|S f|_{0}\right]}{C_{d}}\right]^{4}\right)
$$

with:

$$
\begin{gathered}
C_{13}(k)=C_{12}(k) \\
C_{14}\left[W(f),|S f|_{k-3}\right]=\log \left(1 \vee|S f|_{k-3}\right)+W(f)+1
\end{gathered}
$$

\subsection{Iteration of the reasoning: proof of estimation (5) of theorem} 1.6.

To obtain an estimation of the $C^{k-1-\beta-\eta}$-norm of the conjugacy, we iterate estimation

(41). We take $\gamma_{0}=\gamma_{i}$ and $\gamma_{1}=\gamma_{i+1}=\frac{1}{2}\left(g\left(\gamma_{i}\right)+\gamma_{i}\right)$. Thus, $\gamma_{i+1}<g\left(\gamma_{i}\right)$ and

$\lim _{i \rightarrow+\infty} \gamma_{i}=k-2-\beta$. We need to estimate the rank above which $\gamma_{i} \geq k-2-\beta-\eta$ :

Claim 5.17. Let $C_{77}=\frac{\beta+3 / 2}{\beta+2}, C_{78}=\frac{k-2-\beta}{2(\beta+2)}$. If

$$
n \geq \log \left(\frac{C_{78}}{\eta\left(1-C_{77}\right)}\right) / \log \left(\frac{1}{C_{77}}\right)=C_{79}
$$

we have $\gamma_{n} \geq k-2-\beta-\eta$.

Proof. We have: $\gamma_{n+1}=C_{77} \gamma_{n}+C_{78}$. Therefore,

$\gamma_{n}=C_{78} \frac{1-C_{77}^{n}}{1-C_{77}}$. Therefore, $\left|\gamma_{n}-(k-2-\beta)\right|=\left|C_{78} \frac{C_{77}^{n}}{1-C_{77}}\right| \leq \eta$ if $n \geq C_{79}$.

Claim 5.18. Let $F(x)=e^{c(a+b x)^{4}}$. For any $x, c \geq 1, a, b \geq 4$, and integer $n \geq 1$, we have:

$$
F^{n}(x) \leq e^{(n)} \wedge\left((3+n) c(a+b x)^{4}\right)
$$

Proof. We show this estimate by induction. If $n=1$, this estimate holds. Suppose this estimate holds at rank $n$. We have:

$$
F^{n+1}(x)=F^{n}\left(e^{c(a+b x)^{4}}\right) \leq e^{(n)} \wedge\left((3+n) c\left(a+b e^{c(a+b x)^{4}}\right)^{4}\right)
$$

For any $x \geq 4, e^{x} \geq x^{2}$. Since $c(a+b x)^{4} \geq 4$ and $4 n \geq 3+n$, then:

$$
\begin{gathered}
e^{n c(a+b x)^{4}} \geq\left(n c(a+b x)^{4}\right)^{2} \geq(3+n) c(a+b)^{4} \\
e^{(4+n) c(a+b x)^{4}} \geq(3+n) c(a+b)^{4} e^{4 c(a+b x)^{4}} \geq(3+n) c\left(a+b e^{c(a+b x)^{4}}\right)^{4}
\end{gathered}
$$

Hence the estimate at rank $n+1$. 
We apply proposition 5.1. In claim 5.18, we take $x=\log M^{\prime}$, $a=C_{73}^{f, k}+4 k^{2} \log \left(C_{d}^{-1}\right), b=24 k(k+1), c=C_{72}(k, \beta)$. Let

$$
n_{7}=\left\lceil\frac{\log ((k-2-\beta) / \eta)}{\log (1+1 /(2 \beta+3))}\right\rceil
$$

We have: $C_{79}+1>n_{7} \geq C_{79}$. We get:

$\left\|\log D f^{N}\right\|_{k-2-\beta-\eta} \leq e^{\left(n_{7}\right)} \wedge\left(\left(3+n_{7}\right) C_{72}(k, \beta)\left(C_{73}^{f, k}+4 k^{2} \log \left(C_{d}^{-1}\right)+24 k(k+1) \log M^{\prime}\right)^{4}\right)$

Moreover, by corollary 5.5, we have:

$$
\left\|D f^{N}\right\|_{k-2-\beta-\eta} \leq C_{54}\left(1+\left\|\log D f^{N}\right\|_{k-2-\beta-\eta}\right)
$$

Since $\|D h\|_{k-2-\beta-\eta} \leq\left\|D f^{N}\right\|_{k-2-\beta-\eta}$, we get:

$\|D h\|_{k-2-\beta-\eta} \leq e^{\left(n_{7}\right)} \wedge\left(\left(4+n_{7}\right) C_{72}(k, \beta)\left[C_{73}^{f, k}+4 k^{2} \log \left(C_{d}^{-1}\right)+25 k(k+1) \log M^{\prime}\right]^{4}\right)$

Since $M^{\prime} \leq C_{2}$, we let:

$C_{11}\left[\eta, k, \beta, C_{d},|D f|_{0}, W(f),|S f|_{k-3}\right]=\left(4+n_{7}\right) C_{72}(k, \beta)\left[C_{73}^{f, k}+4 k^{2} \log \left(C_{d}^{-1}\right)+25 k(k+1) \log C_{2}\right]^{4}$

We recall that:

$$
\begin{gathered}
n_{7}=\left\lceil\frac{\log ((k-2-\beta) / \eta)}{\log (1+1 /(2 \beta+3))}\right\rceil \\
C_{72}(k, \beta)=\frac{18 \times 5^{2} \times 8^{2}}{(\log 2)^{3}} \frac{k^{5}}{(\min (k-(2 \beta+1), k-(\beta+2)))^{3}} \\
C_{73}^{f, k} \leq(k+7) !\left(1+W(f)+\log \left(1 \vee|S f|_{k-3}\right)\right)
\end{gathered}
$$

We have:

$\|D h\|_{k-2-\beta-\eta} \leq e^{([\log ((k-2-\beta) / \eta) / \log (1+1 /(2 \beta+3))])} \wedge\left(C_{11}\left[\eta, k, \beta, C_{d},|D f|_{0}, W(f),|S f|_{k-3}\right]\right)$

\section{Appendix: Omitted Proofs}

\subsection{Proof of lemma 4.4}

We follow [14] but we give more details. Let $p \leq q_{n+1}$. The case $r=1$ stems from lemma 4.3. For the case $r=2$, we also use lemma 4.3:

$$
\left|D^{2} \log D f^{p}(x)\right| \leq\left|S f^{p}(x)\right|+\frac{1}{2}\left|D \log D f^{p}(x)\right|^{2} \leq\left(C_{24}^{f}+\frac{1}{2}\left(C_{26}^{f}\right)^{2}\right) \frac{M_{n}}{m_{n}(x)^{2}}
$$

In particular, we can take 


$$
C_{27}^{f}(2)=82|S f|_{0} e^{8 W(f)}
$$

For $r>2$, we prove lemma 4.4 by induction. Suppose the lemma is proved up to $r \geq 2$. Since for any $C^{3}$-diffeomorphisms $g$ and $h$,

$$
S(g \circ h)=(S g \circ h)(D h)^{2}+S h
$$

then for $p \geq 1$,

$$
S f^{p}=\sum_{i=0}^{p-1}\left(S f \circ f^{i}\right)\left(D f^{i}\right)^{2}
$$

and by differentiating this last equality, we get, for $r \geq 0, n \geq 1$,

$$
D^{r} S f^{p}=\sum_{l=0}^{r} \sum_{i=0}^{p-1}\left(D^{r-l} S f \circ f^{i}\right)\left(D f^{i}\right)^{r-l+2} F_{l}^{r}\left(D \log D f^{i}, \ldots, D^{l} \log D f^{i}\right)
$$

where $F_{l}^{r}$ is a polynomial in $l$ variables $X_{1}, \ldots, X_{l}$, homogenous of weight $l$ if $X_{i}$ is given the weight $i$. Moreover, since $S f=D^{2} \log D f-\frac{1}{2}(D \log D f)^{2}$, then for $r \geq 2$,

$$
D^{r-2} S f=D^{r} \log D f+G_{r}\left(D \log D f, \ldots, D^{r-1} \log D f\right)
$$

where $G_{r}$ is a polynomial in $r-1$ variables $X_{1}, \ldots, X_{r-1}$, homogeneous of weight $r$ if $X_{i}$ is given the weight $i$. Therefore, in order to estimate $\left|D^{r} \log D f\right|_{0}$, it suffices to estimate $F_{l}^{r}\left(D \log D f^{i}, \ldots, D^{l} \log D f^{i}\right)$ and $G_{r}\left(D \log D f, \ldots, D^{r-1} \log D f\right)$. These estimations are given by lemmas 6.1 and 6.2. They are used in [14] but we recall them here in order to compute the constants $C_{80}^{f}(r)$ in lemma 6.1 and $C_{81}^{f}(r)$ in lemma 6.2.

Lemma 6.1. Under the induction assumption, for $0 \leq l \leq r$ and $0 \leq p \leq q_{n+1}$, we have:

$$
\left|F_{l}^{r}\left(D \log D f^{p}(x), \ldots, D^{l} \log D f^{p}(x)\right)\right| \leq C_{80}^{f}(r)\left[\frac{M_{n}^{1 / 2}}{m_{n}(x)}\right]^{l}
$$

with:

$$
C_{80}^{f}(r)=(r) ! \frac{(2 r) !}{2}\left(C_{27}^{f}(r)\right)^{r}
$$

Proof. We follow [14]. By derivating equation (69), we get:

$$
\begin{array}{r}
D^{r+1} S f^{p}=\sum_{i=0}^{n-1} \sum_{l=0}^{r}\left(D^{r+1-l} S f \circ f^{i}\right)\left(D f^{i}\right)^{r+1-l+2} F_{l}^{r}\left(D \log D f^{i}, \ldots, D^{l} \log D f^{i}\right)+ \\
\left(D^{r-l} S f \circ f^{i}\right)\left(D f^{i}\right)^{r-l+2}(r-l+2) D \log D f^{i} \\
F_{l}^{r}\left(D \log D f^{i}, \ldots, D^{l} \log D f^{i}\right)+\sum_{j=1}^{l} \frac{\partial F_{l}^{r}}{\partial X_{j}}\left(D \log D f^{i}, \ldots, D^{l} \log D f^{i}\right) D^{j+1} \log D f^{i}\left(D f^{i}\right)^{r-l+2}
\end{array}
$$




$$
\begin{array}{r}
D^{r+1} S f^{p}=\sum_{i=0}^{p-1} \sum_{l=0}^{r}\left(D^{r+1-l} S f \circ f^{i}\right)\left(D f^{i}\right)^{r+1-l+2} F_{l}^{r}\left(D \log D f^{i}, \ldots, D^{l} \log D f^{i}\right)+ \\
\sum_{l=1}^{r+1}\left(D^{r+1-l} S f \circ f^{i}\right)\left(D f^{i}\right)^{r-l+3}(r-l+3) D \log D f^{i} F_{l-1}^{r}\left(D \log D f^{i}, \ldots, D^{l-1} \log D f^{i}\right)+ \\
\sum_{l=1}^{r+1} \sum_{j=2}^{l} \frac{\partial F_{l-1}^{r}}{\partial X_{j-1}}\left(D \log D f^{i}, \ldots, D^{l-1} \log D f^{i}\right) D^{j} \log D f^{i}\left(D f^{i}\right)^{r+1-l+2}
\end{array}
$$

Therefore, for $1 \leq l \leq r$,

$$
F_{l}^{r+1}=F_{l}^{r}+(r-l+3) X_{1} F_{l-1}^{r}+\sum_{j=2}^{l} X_{j} \frac{\partial F_{l-1}^{r}}{\partial X_{j-1}}
$$

for $l=0$,

$$
F_{l}^{r+1}=F_{l}^{r}
$$

and for $l=r+1$,

$$
F_{l}^{r+1}=(r-l+3) X_{1} F_{l-1}^{r}+\sum_{j=2}^{l} X_{j} \frac{\partial F_{l-1}^{r}}{\partial X_{j-1}}
$$

Now, let us write

$$
F_{l}^{r}=\sum_{i_{1}+2 i_{2}+\ldots+l l_{l}=l} a_{l, r}\left(i_{1}, \ldots, i_{l}\right) X_{1}^{i_{1}} \ldots X_{l}^{i_{l}}
$$

We have $a_{l, r}\left(i_{1}, \ldots, i_{l}\right) \geq 0$. Let

$$
a_{l, r}=\max _{i_{1}+2 i_{2}+\ldots+l i_{l}=l} a_{l, r}\left(i_{1}, \ldots, i_{l}\right)
$$

and

$$
\bar{a}_{r}=\max _{0 \leq j \leq r} a_{j, r}
$$

Consider $i_{1}, \ldots, i_{l}$ such that $a_{l, r}\left(i_{1}, \ldots, i_{l}\right)=a_{l, r}$. By applying equation (71), we have, for $1 \leq l \leq r$ :

$a_{l, r+1} \leq a_{l, r}+(r+3-l) a_{l-1, r}+(l-1)\left(\max i_{j}\right) a_{l-1, r} \leq\left(r+3-l+l^{2}-l\right) \bar{a}_{l, r} \leq(r+1)^{2} \bar{a}_{l, r}$

For $l=0$ or $r+1$, this estimate still holds. Therefore, $\bar{a}_{r+1} \leq(r+1)^{2} \bar{a}_{r}$ and by iteration, we obtain:

$$
\bar{a}_{r} \leq(r !)^{2}
$$

Moreover, since

$$
F_{l}^{r}\left(D \log D f^{i}, \ldots, D^{l} \log D f^{i}\right)=\sum_{i_{1}+2 i_{2}+\ldots+l l_{l}=l} a_{l, r}\left(i_{1}, \ldots, i_{l}\right)\left(D \log D f^{i}\right)^{i_{1}} \ldots\left(D^{l} \log D f^{i}\right)^{i_{l}}
$$


and since \#\{(i, $\left.\left.\ldots, i_{l}\right) / i_{1}+2 i_{2}+\ldots+l i_{l}=l\right\} \leq \#\left\{\left(i_{1}, \ldots, i_{l}\right) / i_{1}+i_{2}+\ldots+i_{l}=l\right\}=$ $\frac{(2 l-1) !}{l !(l-1) !}$ (this classical equality can be shown by induction) then by applying the induction assumption,

$\left|F_{l}^{r}\left(D \log D f^{i}(x), \ldots, D^{l} \log D f^{i}(x)\right)\right| \leq(r !)^{2} \frac{(2 l-1) !}{l !(l-1) !} \max _{i_{1}+2 i_{2}+\ldots+l l_{l}=l}\left(C_{27}^{f}(1)\right)^{i_{1}} \ldots\left(C_{27}^{f}(l)\right)^{l i_{l}}\left[\frac{M_{n}^{1 / 2}}{m_{n}(x)}\right]^{l}$

and since the $C_{27}^{f}(i)$ are increasing with $i$, we obtain:

$$
\left|F_{l}^{r}\left(D \log D f^{i}(x), \ldots, D^{l} \log D f^{i}(x)\right)\right| \leq C_{80}^{f}(r)\left[\frac{M_{n}^{1 / 2}}{m_{n}(x)}\right]^{l}
$$

Likewise, the estimation of $G_{r}\left(D \log D f^{p}, \ldots, D^{l-1} \log D f^{p}\right)$ is given by the lemma:

Lemma 6.2. For any $x \in \mathbb{T}^{1}, 0 \leq p \leq q_{n+1}, r \geq 2$,

$$
\left|G_{r}\left(D \log D f^{p}(x), \ldots, D^{l-1} \log D f^{p}(x)\right)\right| \leq C_{81}^{f}(r)\left[\frac{M_{n}^{1 / 2}}{m_{n}(x)}\right]^{r}
$$

with:

$C_{81}^{f}(r+1)=\frac{(2 r) !}{2(r+1)}\left(C_{27}^{f}(r)\right)^{r+1}$

Proof. The polynomial $G_{r}$ satisfies the following identity:

$$
G_{r+1}=\sum_{j=2}^{r} X_{j} \frac{\partial G_{r}}{\partial X_{j-1}}
$$

We denote

$$
G_{r}=\sum_{i_{1}+2 i_{2}+\ldots+(r-1) i_{r-1}=r} b_{r}\left(i_{1}, \ldots, i_{r-1}\right) X_{1}^{i_{1}} \ldots X_{r-1}^{i_{r-1}}
$$

(we have, for example, $G_{2}=-\frac{1}{2} X_{1}^{2}$ )

Let

$$
b_{r}=\max _{i_{1}+2 i_{2}+\ldots+(r-1) i_{r-1}=r}\left|b_{r}\left(i_{1}, \ldots, i_{r-1}\right)\right|
$$

For $r \geq 2$, we have $b_{r+1} \leq r\left(\max _{1 \leq j \leq r-1} i_{j}\right) b_{r} \leq r^{2} b_{r}$ and therefore, $b_{r} \leq \frac{(r-1) !^{2}}{2}$

Therefore,

$\left|G_{r+1}\left(D \log D f^{p}(x), \ldots, D^{r} \log D f^{p}(x)\right)\right| \leq \frac{r !}{2} \frac{(2 r) !}{r !(r+1) !} \max _{i_{1}+2 i_{2}+\ldots+r i_{r}=r+1}\left(C_{27}^{f}(1)\right)^{i_{1}} \ldots\left(C_{27}^{f}(r)\right)^{i_{r}}\left[\frac{M_{n}^{1 / 2}}{m_{n}(x)}\right]^{r+1}$

Since the constants $C_{27}^{f}(r)$ are increasing with $r$, we can take:

$$
C_{81}^{f}(r+1)=\frac{(2 r) !}{2(r+1)}\left(C_{27}^{f}(r)\right)^{r+1}
$$


We can now show estimation (14). By applying equation (70), we have, for $r \geq 2$ :

$$
D^{r+1} \log D f^{p}=D^{r-1} S f^{p}-G_{r+1}\left(D \log D f^{p}, \ldots, D^{r} \log D f^{p}\right)
$$

Therefore, by equation (69) and lemma 4.1,

$$
\begin{gathered}
\left|D^{r+1} \log D f^{p}(x)\right| \leq\left(r C_{80}^{f}(r)|S f|_{r-1} e^{(r+1) W(f)}+C_{81}^{f}(r+1)\right)\left(\frac{M_{n}^{1 / 2}}{m_{n}(x)}\right)^{r+1} \\
\left|D^{r+1} \log D f^{p}(x)\right| \leq\left(C_{27}^{f}(r)\right)^{r} \frac{(2 r) !}{2}\left(|S f|_{r-1} e^{(r+1) W(f)}+C_{27}^{f}(r)\right)\left(\frac{M_{n}^{1 / 2}}{m_{n}(x)}\right)^{r+1}
\end{gathered}
$$

We can show by induction on $r$ that we can take, for $r \geq 3$,

$$
C_{27}^{f}(r)=\left[C_{27}^{f}(2)(2 r)^{2 r}\left(\max \left(1,|S f|_{r-2}\right)\right) e^{r W(f)}\right]^{r !}
$$

\subsection{Proof of proposition 5.3}

This estimation is well-known (see e.g. the appendix in [6]). We recall the proof in order to determine the constant. We write $\gamma_{2}=r_{2}+\gamma_{2}^{\prime}, \gamma_{3}=r_{3}+\gamma_{3}^{\prime}$ with $0<\gamma_{2}^{\prime}, \gamma_{3}^{\prime}<1$, and we estimate $\left|D^{r_{2}} \phi\right|_{\gamma_{2}^{\prime}}$ in function of $\left|D^{r_{3}} \phi\right|_{\gamma_{3}^{\prime}}$ and $|\phi|_{0}$. We use [5, p. 110]:

Proposition 6.3. Let $0<\beta<1$ and $\phi \in C^{1}\left(\mathbb{T}^{1}\right)$. We have:

$$
|\phi|_{\beta} \leq 2|\phi|_{0}^{1-\beta}|D \phi|_{0}^{\beta}
$$

Proposition 6.4. Let $0<\beta<1$ and $\phi \in C^{\beta}\left(\mathbb{T}^{1}\right)$. If $\beta^{\prime}<\beta$, we have:

$$
|\phi|_{\beta^{\prime}} \leq 2|\phi|_{0}^{1-\frac{\beta^{\prime}}{\beta}}|\phi|_{\beta}^{\frac{\beta^{\prime}}{\beta}}
$$

Proposition 6.5. Let $0<\beta \leq 1$ and $\phi \in C^{1}\left(\mathbb{T}^{1}\right)$ such that $D \phi \in C^{\beta}\left(\mathbb{T}^{1}\right)$. We have:

$$
|D \phi|_{0} \leq \frac{3}{2} 2^{\frac{\beta}{1+\beta}}\left(|\phi|_{0}^{\beta}|D \phi|_{\beta}\right)^{\frac{1}{1+\beta}}
$$

Moreover, for $\phi \in C^{2}\left(\mathbb{T}^{1}\right)$, we have:

$$
|D \phi|_{0} \leq\left(2|\phi|_{0}\left|D^{2} \phi\right|_{0}\right)^{1 / 2}
$$

First, we suppose $\gamma_{2}^{\prime}=\gamma_{3}^{\prime}=0$. We already know that for any $j<l$, there exists $C_{82}(j, l)$ such that:

$$
\left|D^{j} \phi\right|_{0} \leq C_{82}(j, l)|\phi|_{0}^{1-\frac{j}{l}}\left|D^{l} \phi\right|_{0}^{\frac{j}{l}}
$$

We want to estimate a possible $C_{82}(j, l)$ in function of $l$. First, we show that $C_{82}(1, l)=C_{82}(l-1, l)=(\sqrt{2})^{l-1}$. We proceed inductively on $l$. For $l=2$, by proposition $6.5, C_{82}(1, l)=C_{82}(l-1, l)=\sqrt{2}$. For $l \geq 3$, we have:

$$
\begin{gathered}
|D \phi|_{0} \leq C_{82}(1, l-1)|\phi|_{0}^{\frac{l-2}{l-1}}\left|D^{l-1} \phi\right|_{0}^{\frac{1}{l-1}} \\
\left|D^{l-2} \phi\right|_{0} \leq C_{82}(l-2, l-1)|\phi|_{0}^{\frac{1}{l-1}}\left|D^{l-1} \phi\right|_{0}^{\frac{l-2}{l-1}}
\end{gathered}
$$


By applying (73) to $D \phi$, we also have:

$$
\left|D^{l-1} \phi\right|_{0} \leq C_{82}(l-2, l-1)|D \phi|_{0}^{\frac{1}{l-1}}\left|D^{l} \phi\right|_{0}^{\frac{l-2}{l-1}}
$$

By combining this estimate with (72), we get:

$$
\left|D^{l-1} \phi\right|_{0}^{1-\frac{1}{(l-1)^{2}}} \leq C_{82}(l-2, l-1)\left(C_{82}(1, l-1)\right)^{\frac{1}{l-1}}|\phi|_{0}^{\frac{l-2}{(-1)^{2}}}\left|D^{l} \phi\right|_{0}^{\frac{l-2}{-1-1}}
$$

Therefore,

$$
C_{82}(l-1, l)=\left(C_{82}(l-2, l-1)\left(C_{82}(1, l-1)\right)^{\frac{1}{1-1}}\right)^{\frac{1}{1-\frac{1}{(l-1)^{2}}}}
$$

Likewise, we can show that

$$
C_{82}(1, l)=\left(C_{82}(1, l-1)\left(C_{82}(l-2, l-1)\right)^{\frac{1}{l-1}}\right)^{\frac{1}{1-\frac{1}{(l-1)^{2}}}}
$$

Let $u_{l}=\log C_{82}(1, l), v_{l}=\log C_{82}(l-1, l), a_{l}=\frac{1}{1-\frac{1}{(l-1)^{2}}}$. We have:

$$
\begin{aligned}
& u_{l}=a_{l}\left(u_{l-1}+\frac{1}{l-1} v_{l-1}\right) \\
& v_{l}=a_{l}\left(v_{l-1}+\frac{1}{l-1} u_{l-1}\right)
\end{aligned}
$$

Let $w_{l}=u_{l}+v_{l}$. We have: $w_{l}=\frac{l-1}{l-2} w_{l-1}$ and therefore, $w_{l}=(l-1) w_{2}$. Moreover, let $x_{l}=u_{l}-v_{l}$. We have: $x_{l}=\frac{l}{l-1} x_{l-1}$. Since $x_{2}=0$, then $x_{l}=0$ for any $l \geq 3$. Therefore, $u_{l}=v_{l}=(l-1) \log \sqrt{2}$. Therefore, $C_{82}(1, l)=C_{82}(l-1, l)=(\sqrt{2})^{l-1}$.

To show that $C_{82}(j, l) \leq(\sqrt{2})^{l^{2}}$ for $l-2 \geq j \geq 2$, we proceed inductively on l. We know that $C_{82}(1,2)=\sqrt{2}$ and we suppose that $C_{82}(j, l-1) \leq(\sqrt{2})^{(l-1)^{2}}$ for $j=2, \ldots, l-2$. We observe that:

$$
\left|D^{j+1} \phi\right|_{0} \leq C_{82}(j, l-1)|D \phi|_{0}^{1-\frac{j}{l-1}}\left|D^{l} \phi\right|_{0}^{\frac{j}{l-1}}
$$

Therefore,

$$
C_{82}(j+1, l) \leq C_{82}(j, l-1)\left(C_{82}(1, l)\right)^{\frac{l-j-1}{l-1}}=C_{82}(j, l-1)(\sqrt{2})^{l-j-1}
$$

By applying the induction assumption, we get:

$$
C_{82}(j+1, l) \leq(\sqrt{2})^{(l-1)^{2}+l-1} \leq(\sqrt{2})^{l^{2}}
$$

Hence the proposition for $\gamma_{2}, \gamma_{3}$ integers.

Now, we suppose that $\gamma_{2}$ and $\gamma_{3}$ are not integers.

If $r_{2}<r_{3}$, we have:

$$
\left|D^{r_{2}} \phi\right|_{\gamma_{2}^{\prime}} \leq 2\left|D^{r_{2}} \phi\right|_{0}^{1-\gamma_{2}^{\prime}}\left|D^{r_{2}+1} \phi\right|_{0}^{\gamma_{2}^{\prime}}
$$

Moreover, by the interpolation inequality in the integer case, we have: 


$$
\begin{aligned}
\left|D^{r_{2}+1} \phi\right|_{0} & \leq C_{82}\left(r_{3}\right)|\phi|_{0}^{1-\frac{r_{2}+1}{r_{3}}}\left|D^{r_{3}} \phi\right|_{0}^{\frac{r_{2}+1}{r_{3}}} \\
\left|D^{r_{2}} \phi\right|_{0} & \leq C_{82}\left(r_{3}\right)|\phi|_{0}^{1-\frac{r_{2}}{r_{3}}}\left|D^{r_{3}} \phi\right|_{0}^{r_{2}}
\end{aligned}
$$

Therefore, by proposition 6.3

$$
\left|D^{r_{2}} \phi\right|_{\gamma_{2}^{\prime}} \leq 2 C_{82}\left(r_{3}\right)|\phi|_{0}^{\left(1-\gamma_{2}^{\prime}\right)\left(1-\frac{r_{2}}{r_{3}}\right)+\gamma_{2}^{\prime}\left(1-\frac{r_{2}+1}{r_{3}}\right)}\left|D^{r_{3}} \phi\right|_{0}^{\left(1-\gamma_{2}^{\prime}\right) \frac{r_{2}}{r_{3}}+\gamma_{2}^{\prime} \frac{r_{2}+1}{r_{3}}}
$$

On the other hand, by proposition 6.5 ,

$$
\left|D^{r_{3}} \phi\right|_{0} \leq 3\left|D^{r_{3}-1} \phi\right|_{0}^{\frac{\gamma_{3}^{\prime}}{1+\gamma_{3}^{\prime}}}\left|D^{r_{3}} \phi\right|_{\gamma_{3}^{\prime}}^{\frac{1}{1+\gamma_{3}^{\prime}}}
$$

and we also have:

$$
\left|D^{r_{3}-1} \phi\right|_{0} \leq C_{82}\left(r_{3}\right)||_{0}^{\frac{1}{r_{3}}}\left|D^{r_{3}} \phi\right|_{0}^{\frac{r_{3}-1}{r_{3}}}
$$

Therefore,

$$
\left|D^{r_{3}} \phi\right|_{0}^{1-\left(1-\frac{1}{r_{3}}\right)} \frac{\frac{\gamma}{3}_{1}^{\prime}}{1+\gamma_{3}^{\prime}} \leq 3\left(C_{82}\left(r_{3}\right)\right)^{\frac{\gamma_{3}^{\prime}}{1+\gamma_{3}^{\prime}}}|\phi|_{0}^{\left(\frac{\gamma_{3}^{\prime}}{1+\gamma_{3}^{\prime}}\right) \frac{1}{r_{3}}}\left|D^{r_{3}} \phi\right|_{\gamma_{3}^{\prime}}^{\frac{1}{1+\gamma_{3}^{\prime}}}
$$

Therefore,

$$
\left|D^{r_{3}} \phi\right|_{0} \leq 3^{\frac{1+\gamma_{3}^{\prime}}{1+\gamma_{3} / r_{3}}}\left(C_{82}\left(r_{3}\right)\right)^{\frac{\gamma_{3}^{\prime}}{1+\gamma_{3}^{\prime} / r_{3}}}|\phi|_{0}^{\left(\frac{\gamma_{3}^{\prime} / r_{3}}{1+\gamma_{3}^{\prime} / r_{3}}\right) \frac{1}{r_{3}}}\left|D^{r_{3}} \phi\right|_{\gamma_{3}^{\frac{1}{1+\gamma_{3}^{\prime} / r_{3}}}}^{\frac{1}{3}}
$$

Therefore,

$$
\left|D^{r_{2}} \phi\right|_{\gamma_{2}^{\prime}} \leq C_{82}\left(\gamma_{3}\right)|\phi|_{0}^{1-\frac{\gamma_{2}}{\gamma_{3}}}\left|D^{r_{3}} \phi\right|_{\gamma_{3}^{\prime}}^{\frac{\gamma_{2}}{\gamma_{3}}}
$$

with $C_{82}\left(\gamma_{3}\right) \leq 3^{2} C_{82}\left(r_{3}\right) \leq 2^{\left(r_{3}+1\right)^{2}}$ if $r_{3} \geq 2$ and $C_{82}\left(\gamma_{3}\right) \leq 2^{\left(r_{3}+1\right)^{2}+1}$ if $r_{3}=1$.

Finally, if $r_{2}=r_{3}=r$ and $\gamma_{2}^{\prime}<\gamma_{3}^{\prime}$, then

$$
\left|D^{r} \phi\right|_{\gamma_{2}^{\prime}} \leq 2\left|D^{r} \phi\right|_{0}^{1-\frac{\gamma_{2}^{\prime}}{\gamma_{3}}}\left|D^{r} \phi\right|_{\gamma_{3}^{\prime}}^{\frac{\gamma_{2}^{\prime}}{\gamma_{3}}}
$$

By applying estimation (74), we still have:

$$
\left|D^{r_{2}} \phi\right|_{\gamma_{2}^{\prime}} \leq C_{82}\left(\gamma_{3}\right)|\phi|_{0}^{1-\frac{\gamma_{2}}{\gamma_{3}}}\left|D^{r_{3}} \phi\right|_{\gamma_{3}^{\prime}}^{\frac{\gamma_{2}}{\gamma_{3}}}
$$

with $C_{82}\left(\gamma_{3}\right) \leq 2^{\left(r_{3}+1\right)^{2}}$ if $r_{3} \geq 2$ and $C_{82}\left(\gamma_{3}\right) \leq 2^{\left(r_{3}+1\right)^{2}+1}$ if $r_{3}=1$.

This completes the proof of proposition 5.3. 


\subsection{Proof of lemma 5.6.}

If $\gamma<1$, we prove the lemma directly. We prove lemma 5.6 for $\gamma \geq 1$. This is necessary to compute the constant. We write $\gamma=r+\gamma^{\prime}$ with $r$ integer and $0 \leq \gamma^{\prime}<1$. We need the Faa-di-Bruno formula. We first recall this formula and a related property (lemma 6.6). After that, we prove the lemma.

The Faa-di-Bruno formula states that for any integer $u \geq 1$ and functions $f$ and $g$ of class $C^{u}$,

$$
D^{u}[f(g(x))]=\sum_{j=0}^{u} D^{j} f(g(x)) B_{u, j}\left(D g(x), D^{2} g(x), \ldots, D^{(u-j+1)} g(x)\right)
$$

where $B_{u, j}$ are the Bell polynomials, given by:

$$
B_{u, j}\left(x_{1}, x_{2}, \ldots, x_{u-j+1}\right)=\sum \frac{u !}{l_{1} ! l_{2} ! \cdots l_{u-j+1} !}\left(\frac{x_{1}}{1 !}\right)^{l_{1}}\left(\frac{x_{2}}{2 !}\right)^{l_{2}} \cdots\left(\frac{x_{u-j+1}}{(u-j+1) !}\right)^{l_{u-j+1}}
$$

The sum extends over all sequences $l_{1}, l_{2}, l_{3}, \ldots, l_{u-j+1}$ of non-negative integers such that $l_{1}+l_{2}+\ldots=j$ and $l_{1}+2 l_{2}+3 l_{3}+\ldots=u$. The cardinal of this set of sequences is less than $\#\left\{\left(i_{1}, \ldots, i_{l}\right) / i_{1}+i_{2}+\ldots+i_{l}=l\right\}=\frac{(2 l-1) !}{l !(l-1) !}$ (see page 51$)$.

We obtain the estimation, for any $x$, and $u \geq 1$ :

$$
\left|B_{u, j}\left(D g(x), D^{2} g(x), \ldots, D^{(u-j+1)} g(x)\right)\right| \leq \frac{(2 u-1) !}{(u-1) !}\|D g\|_{u-1}^{j}
$$

We also need the lemma:

Lemma 6.6. Let $u \geq 1$ and $u \geq j \geq 0$ be integers. Let $a_{1}, \ldots, a_{u-j+1}, x_{1}, \ldots, x_{u-j+1}$ be real numbers, let $a \geq \max \left\{\left|a_{k}\right| ; 1 \leq k \leq u-j+1\right\}, x \geq \max \left\{\left|x_{k}\right| \vee 1 ; 1 \leq k \leq u-j+1\right\}$. Suppose $x+a \geq 1$. Let $B_{u, j}$ be a Bell polynomial. We have :

$$
\left|B_{u, j}\left(x_{1}+a_{1}, \ldots, x_{u-j+1}+a_{u-j+1}\right)-B_{u, j}\left(x_{1}, \ldots, x_{u-j+1}\right)\right| \leq u^{2} \frac{(2 u) !}{2} a(x+a)^{u}
$$

Proof. Let $u+1 \geq p \geq 1$ and $l_{1}, \ldots, l_{p}$ be integers. We have:

$$
\begin{aligned}
& \left(x_{1}+a_{1}\right)^{l_{1}} \ldots\left(x_{p}+a_{p}\right)^{l_{p}}-x_{1}^{l_{1}} \ldots x_{p}^{l_{p}}=\sum_{i=1}^{p} x_{1}^{l_{1}} \ldots x_{i-1}^{l_{i-1}}\left(x_{i}+a_{i}\right)^{l_{i}} \ldots\left(x_{p}+a_{p}\right)^{l_{p}}-x_{1}^{l_{1}} \ldots x_{i}^{l_{i}}\left(x_{i+1}+a_{i+1}\right)^{l_{i+1}} \ldots\left(x_{p}+a_{p}\right)^{l_{p}} \\
& \left(x_{1}+a_{1}\right)^{l_{1}} \ldots\left(x_{p}+a_{p}\right)^{l_{p}}-x_{1}^{l_{1}} \ldots x_{p}^{l_{p}}=\sum_{i=1}^{p} x_{1}^{l_{1}} \ldots x_{i-1}^{l_{i-1}}\left(x_{i+1}+a_{i+1}\right)^{l_{i+1}} \ldots\left(x_{p}+a_{p}\right)^{l_{p}}\left[\left(x_{i}+a_{i}\right)^{l_{i}}-x_{i}^{l_{i}}\right]
\end{aligned}
$$

(with the conventions $x_{1}^{l_{1}} \ldots x_{0}^{l_{0}}=1$ and $x_{p+1}^{l_{p+1}} \ldots x_{p}^{l_{p}}=1$ ).

Since $\left|\left(x_{i}+a_{i}\right)^{l_{i}}-x_{i}^{l_{i}}\right| \leq l_{i}\left|a_{i}\right|\left(\left|x_{i}\right|+\left|a_{i}\right|\right)^{l_{i}-1} \leq l_{i} a(x+a)^{l_{i}-1}, l_{i} \leq u$ and $x+a \geq 1$, we obtain:

$\left|B_{u, j}\left(x_{1}+a_{1}, \ldots, x_{u-j+1}+a_{u-j+1}\right)-B_{u, j}\left(x_{1}, \ldots, x_{u-j+1}\right)\right| \leq a(u-j+1) u B_{u, j}(x+a, \ldots, x+a)$

The formula giving the Bell polynomials implies:

$$
B_{u, j}(x+a, \ldots, x+a) \leq \frac{(2 u) !}{2}(x+a)^{u}
$$


For any $1 \leq u \leq r$,

$$
\mid D^{u}\left(\phi(\psi(x)) \mid \leq(u+1)\|D \phi\|_{r-1} \frac{(2 u) !}{2}\|D \psi\|_{u-1}^{u}\right.
$$

We estimate $\left|D^{r}(\phi \circ \psi)\right|_{\gamma^{\prime}}$. The Faa-di-Bruno formula applied to $\phi \circ \psi$ gives:

$$
\begin{gathered}
\left|D^{r}[\phi(\psi(x))]-D^{r}[\phi(\psi(y))]\right| \leq \sum_{j=0}^{r}\left|D^{j} \phi(\psi(x))\right| \\
\left|B_{r, j}\left(D \psi(x), \ldots, D^{(r-j+1)} \psi(x)\right)-B_{r, j}\left(D \psi(y), \ldots, D^{(r-j+1)} \psi(y)\right)\right|+ \\
\left|D^{j} \phi(\psi(x))-D^{j} \phi(\psi(y))\right|\left|B_{r, j}\left(D \psi(y), \ldots, D^{(r-j+1)} \psi(y)\right)\right|
\end{gathered}
$$

Moreover, for $1 \leq j \leq r,\left|D^{j} \phi(\psi(x))\right| \leq\|D \phi\|_{r-1}$, and

$$
\left|D^{j} \phi(\psi(x))-D^{j} \phi(\psi(y))\right| \leq\left|D^{j} \phi\right|_{\gamma^{\prime}}|\psi(x)-\psi(y)|^{\gamma^{\prime}} \leq\|D \phi\|_{\gamma-1}|D \psi|_{0}^{\gamma^{\prime}}|x-y|^{\gamma^{\prime}}
$$

Estimation (75) gives:

$$
\left|B_{r, j}\left(D \psi(y), D^{2} \psi(y), \ldots, D^{(r-j+1)} \psi(y)\right)\right| \leq \frac{(2 r !)}{2}\|D \psi\|_{r-1}^{r}
$$

We apply lemma 6.6: let $x_{i}=D^{i} \psi(x)$ and $a_{i}=D^{i} \psi(y)-D^{i} \psi(x)$. Let $x=\|D \psi\|_{r-1}$ and $a=\max _{1 \leq i \leq r-j+1}\left|D^{i} \psi(x)-D^{i} \psi(y)\right|$. We have:

$\left|B_{r, j}\left(D \psi(x), \ldots, D^{(r-j+1)} \psi(x)\right)-B_{r, j}\left(D \psi(y), \ldots, D^{(r-j+1)} \psi(y)\right)\right| \leq r^{2} \frac{(2 r !)}{2}\left(2\|D \psi\|_{\gamma-1}\right)^{r} \max _{1 \leq i \leq r-j+1}\left|D^{i} \psi(x)-D^{i} \psi(y)\right|$

$\left|B_{r, j}\left(D \psi(x), \ldots, D^{(r-j+1)} \psi(x)\right)-B_{r, j}\left(D \psi(y), \ldots, D^{(r-j+1)} \psi(y)\right)\right| \leq r^{2} 2^{r} \frac{(2 r !)}{2}\|D \psi\|_{\gamma-1}^{r+1}|x-y|^{\gamma^{\prime}}$

Therefore, we get:

$$
\left|D^{r}(\phi \circ \psi)\right|_{\gamma^{\prime}} \leq\|D \phi\|_{\gamma-1}\|D \psi\|_{\gamma-1}^{\lfloor\jmath+1} \frac{(2 r) !}{2}(r+1)\left(r^{2} 2^{r}+1\right) \leq\|D \phi\|_{\gamma-1}\|D \psi\|_{\gamma-1}^{L \jmath+1}((2 r) !)^{2}
$$

\subsection{Estimates on some polynomials}

Lemma 5.4 is used for some specific polynomials. There exist $A_{l}, B_{l}, G_{l}^{r}, E_{l}^{r}$, polynomials of $l$ variables $X_{1}, \ldots, X_{l}$ homogeneous of weight $l$ if $X_{i}$ has weight $i$, such that, for $l \geq 1$, and for any diffeomorphisms $g$ and $h$ sufficiently differentiable, we have [14, p. 337-338]:

$$
\begin{gathered}
D^{l+1} g=A_{l}\left(D \log D g, \ldots, D^{l} \log D g\right) D g \\
D^{l} \log D g=B_{l}\left(\frac{D^{2} g}{D g}, \ldots, \frac{D^{l+1} g}{D g}\right)
\end{gathered}
$$

For $r \geq 0$, 
$D^{r} \log D(g \circ h)=\left(D^{r} \log D g \circ h\right)(D h)^{r}+D^{r} \log D h+\sum_{l=1}^{r-1} D^{r-l} \log D g \circ h(D h)^{r-l} G_{l}^{r}\left(D \log D h, \ldots, D^{l} \log D h\right)$

For $r \geq 0$ and $n \geq 1$,

$$
D^{r} \log D g^{n}=\sum_{l=0}^{r-1} \sum_{i=0}^{n-1}\left(D^{r-l} \log D g \circ g^{i}\right)\left(D g^{i}\right)^{r-l} E_{l}^{r}\left(D \log D g^{i}, \ldots, D^{l} \log D g^{i}\right)
$$

Lemma 5.4 uses the following estimate:

Claim 6.7. Let $P=A_{l}, B_{l}, G_{l}^{r}$ or $E_{l}^{r}$. Write $P=\sum_{\sum_{k=1}^{l} k j_{k}=l} a_{j_{i}, \ldots, j_{l}} X_{1}^{j_{1}} \ldots X_{l}^{j_{l}}$. We have:

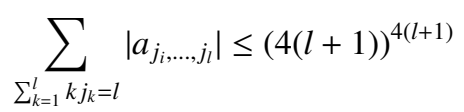

Proof. For example, if $P=B_{l}$, we have

$$
B_{l+1}\left(\frac{D^{2} g}{D g}, \ldots, \frac{D^{l+2} g}{D g}\right)=D B_{l}\left(\frac{D^{2} g}{D g}, \ldots, \frac{D^{l+1} g}{D g}\right)
$$

Since

$$
D\left(\frac{D^{u} g}{D g}\right)=\frac{D^{u+1} g}{D g}-\frac{D^{u} g}{D g} \frac{D^{2} g}{D g}
$$

then

$$
B_{l+1}=\sum_{i=1}^{l}\left(X_{i+1}-X_{i} X_{1}\right) \frac{\partial B_{l}}{\partial X_{i}}
$$

Let $b_{l}=\max \left|a_{j_{i}, \ldots, j_{l}}\right|$. We have: $b_{l+1} \leq 2 l^{2} b_{l}$. Thus $b_{l} \leq 2^{l}((l-1) !)^{2}$. Moreover, the sum $\sum_{\sum_{k=1}^{l} k j_{k}=l}\left|a_{j_{i}, \ldots, j_{l}}\right|$ has less than $\frac{(2 l-1) !}{l !(l-1) !}$ terms. Hence the claim for $P=B_{l}$. The computations are analogous for the other polynomials (and analogous to the proof of lemma 4.4 in appendix 6.1).

To obtain lemma 5.4, we apply estimations (47) and (48) to each monomial of $P$, and we apply this claim.

\section{References}

[1] VI Arnol'd. Small denominators I: Mappings of the circumference into itself. American Mathematical Society Translations, 46:213-284, 1965.

[2] M Benhenda. Circle Diffeomorphisms: Quasi-reducibility and Commuting Diffeomorphisms. submitted.

[3] R. De la Llave. A tutorial on KAM theory. In Smooth Ergodic Theory and Its Applications: Proceedings of the AMS Summer Research Institute on Smooth Ergodic Theory and Its Applications, July 26-August 13, 1999, University of Washington, Seattle, volume 69, page 175. Amer Mathematical Society, 1999. 
[4] B. Fayad and K. Khanin. Smooth linearization of commuting circle diffeomorphisms. Annals of Mathematics, 170:961-980, 2009.

[5] M.R. Herman. Sur la Conjugaison Différentiable des Difféomorphismes du Cercle a des Rotations. Publications Mathématiques de L'IHÉS, 49(1):5-233, 1979.

[6] L. Hormander. The boundary problems of physical geodesy. Archive for Rational Mechanics and Analysis, 62(1):1-52, 1976.

[7] A. Katok and B. Hasselblatt. Introduction to the modern theory of dynamical systems, volume 54. Cambridge University Press, 1996.

[8] Y. Katznelson and D. Ornstein. The absolute continuity of the conjugation of certain diffeomorphisms of the circle. Ergodic Theory Dynamical Systems, 9(4):681690, 1989.

[9] Y. Katznelson and D. Ornstein. The differentiability of the conjugation of certain diffeomorphisms of the circle. Ergodic Theory Dynamical Systems, 9(4):643$680,1989$.

[10] K. Khanin and A. Teplinsky. Herman's theory revisited. Inventiones Mathematicae, 178(2):333-344, 2009.

[11] KM Khanin and Y.G. Sinai. A new proof of M. Herman's theorem. Communications in Mathematical Physics, 112(1):89-101, 1987.

[12] J. Moser. A rapidly convergent iteration method and non-linear partial differential equations. i. Ann. Scuola Norm. Sup. Pisa (3), 20:265-315, 1966.

[13] Y.G. Sinai and KM Khanin. Smoothness of conjugacies of diffeomorphisms of the circle with rotations. Russian Mathematical Surveys, 44(1):69-99, 1989.

[14] J.C. Yoccoz. Conjugaison différentiable des difféomorphismes du cercle dont le nombre de rotation vérifie une condition diophantienne. Annales scientifiques de l'École Normale Supérieure Sér. 4, 17(3):333-359, 1984.

[15] J.C. Yoccoz. Analytic linearization of circle diffeomorphisms. Dynamical systems and small divisors, pages 125-173, 2002. 